\title{
MCPH1 inhibits condensin II during interphase by regulating its SMC2-kleisin interface
}

Martin Houlard ${ }^{\dagger 1}$, Erin E. Cutts ${ }^{\dagger 2}$, Muhammad S. Shamim ${ }^{3,4,5}$, Jonathan Godwin ${ }^{1}$, David Weisz ${ }^{3,5}$, Aviva Presser Aiden ${ }^{3,5}$, Erez Lieberman Aiden ${ }^{3,5}$, Lothar

Schermelleh $^{1}$, Alessandro Vannini ${ }^{2,6^{*}}$, Kim Nasmyth ${ }^{1^{*}}$

†These authors contributed equally.

1. Department of Biochemistry, University of Oxford, United Kingdom

2. Division of Structural Biology, The Institute of Cancer Research, London, United Kingdom.

3. The Center for Genome Architecture, Department of Molecular and Human Genetics, Baylor College of Medicine, Houston, TX 77030

4. Medical Scientist Training Program, Baylor College of Medicine, Houston, TX 77030, USA. 4 Department of Bioengineering, Rice University, Houston, TX 77030 , USA.

5. Center for Theoretical Biological Physics, Rice University, Houston, TX 77005, USA.

6. Fondazione Human Technopole, Structural Biology Research Centre, Milan, Italy

*To whom correspondence should be addressed: ashley.nasmyth@bioch.ox.ac.uk and alessandro.vannini@fht.org 


\section{ABSTRACT:}

The dramatic change in morphology of chromosomal DNAs between interphase and mitosis is one of the defining features of the eukaryotic cell cycle. Two types of enzymes, namely cohesin and condensin confer the topology of chromosomal DNA by extruding DNA loops. While condensin normally configures chromosomes exclusively during mitosis, cohesin does so during interphase. The processivity of cohesin's LE during interphase is limited by a regulatory factor called WAPL, which induces cohesin to dissociate from chromosomes via a mechanism that requires dissociation of its kleisin from the neck of SMC3. We show here that a related mechanism may be responsible for blocking condensin II from acting during interphase. Cells from patients carrying mutations in the Mcph1 gene undergo premature chromosome condensation but it has never been established for certain whether MCPH1 regulates condensin II directly. We show that deletion of Mcph1 in mouse embryonic stem cells unleashes an activity of condensin II that triggers formation of compact chromosomes in G1 and G2 phases, which is accompanied by enhanced mixing of $A$ and $B$ chromatin compartments, and that this occurs even in the absence of CDK1 activity. Crucially, inhibition of condensin II by MCPH1 depends on the binding of a short linear motif within MCPH1 to condensin II's NCAPG2 subunit. We show that the activities of both Cohesin and Condensin II may be restricted during interphase by similar types of mechanisms as MCPH1's ability to block condensin II's association with chromatin is abrogated by the fusion of SMC2 with NCAPH2. Remarkably, in the absence of both WAPL and MCPH1, cohesin and condensin II transform chromosomal DNAs of G2 cells into chromosomes with a solenoidal axis showing that both SMC complexes must be tightly regulated to adjust both the chromatid's structure and their segregation. 
52

\section{INTRODUCTION}

The segregation of sister DNAs during mitosis requires that they are first disentangled and organised into individual sister chromatids before being pulled in opposite directions by the mitotic spindle upon the dissolution of sister chromatid cohesion by separase. Chromatid formation during mitosis and sister chromatid cohesion necessary for bi-orientation are mediated by highly related structural maintenance of chromosomes (SMC)-kleisin complexes, namely condensin and cohesin respectively. In both cases, the activity of their ring-like SMC-kleisin trimers is regulated by large hook-shaped proteins composed of tandem HEAT repeats, known as HAWKs (HEAT repeat proteins associated with kleisins) ${ }^{1}$.

The conundrum that complexes with such similar geometries appear to perform such dissimilar functions has been resolved by the realisation that cohesin also organises DNAs into chromatid-like structures, albeit during interphase and only upon ablation of a regulatory protein called WAPL ${ }^{2}$ as well as naturally during meiotic prophase ${ }^{3}$ or during $\mathrm{V}(\mathrm{D}) \mathrm{J}$ recombination when WAPL is downregulated in pro-B cells 4. There is now considerable evidence that cohesin and condensin organise chromosomal DNAs during interphase and mitosis, respectively, by extruding DNA loops in a processive manner. Indeed, both exhibit such loop extrusion (LE) activity in vitro $^{5,6}$. LE mediated by cohesin is halted or at least retarded at specific sequences bound by CTCF, and the processivity of the complex is reduced by WAPL which induces cohesin's dissociation from chromatin, albeit only infrequently every 10-20 $\min { }^{7-9}$. No such site-specific DNA binding proteins are known to regulate condensin. In both cases, chromatid formation is envisaged to arise from processive LE activity, 
77 which organises DNA into a series of loops, each emanating from a central core containing the SMC-kleisin loop extruders ${ }^{1}$.

Mammalian cells possess two types of condensin complexes: condensin I and II. Both complexes share the same SMC proteins, SMC2 and SMC4, which both contain $50 \mathrm{~nm}$ long anti-parallel coiled coils connecting a hinge domain at one end with an ATPase domain, formed from the $\mathrm{N}$ - and $\mathrm{C}$-terminal extremities, at the other. Dimerisation via their hinges creates V-shaped SMC2/4 heterodimers whose ATPase heads are inter-connected by their kleisin subunits, NCAPH1 for condensin I and NCAPH2 for condensin II. These in turn recruit different HAWK regulatory subunits, NCAPD2 or D3 and NCAPG or G2 for condensin I and II respectively ${ }^{10}$. While condensin II remains in the nucleus during interphase and starts to organise chromosomal DNAs during prophase, condensin I only has access to the DNA after nuclear envelope break down ${ }^{11-13}$. Despite this difference, both accumulate along the longitudinal axes of metaphase chromosomes ${ }^{13,14}$. HiC data suggest that condensin I extrudes shorter loops that are nested within longer ones previously created by condensin II. Interactions between distant loops suggest that the loops created by condensin II may be organised radially around a coiled or solenoidal axis ${ }^{15}$. The ratio between condensin I and condensin II adjusts the coiling of chromosome axes, partly by altering the width of the central spiral and generating curled chromosomes ${ }^{16}$. An important factor in limiting the processivity of loop extrusion by cohesin is its release from chromatin. This process that depends on WAPL involves engagement of cohesin's ATPase heads ${ }^{17}$ and dissociation of the $\mathrm{N}$-terminal domain (NTD) of its SCC1 kleisin subunit from the coiled coil (neck) that emerges from SMC3's ATPase head domain ${ }^{18}$. Because a co-translational fusion of SCC1's NTD to the C-terminal domain of SMC3's ATPase blocks release, it has been proposed that it involves 
102 passage of DNAs previously entrapped inside its SMC-kleisin ring through an exit gate created by dissociation of SCC1 from SMC3 ${ }^{19,20}$. Indeed, the kleisin NTDs appear to

104 dissociate in vitro from engaged SMC2 and SMC4 heads ${ }^{21,22}$ as well as those of

105 SMC1 and SMC3 $19,23,24$. This observation raises the possibility that release via a

106 kleisin-SMC exit gate might be a feature of chromosomal condensin complexes as

107 well as cohesin. However, whether release of this nature occurs in vivo and whether

108 it has an important function is not known.

109 Condensin II's activity and functions within the nuclei of interphase cells are

110 poorly understood. While some studies have reported that it binds to chromatin during

111 interphase ${ }^{25}$, its depletion from post mitotic mouse liver cells has little or no effect on

112 genome organisation or transcription ${ }^{26}$. Likewise, the notion that condensin II is

113 activated as cells enter mitosis via kinase cascade involving CDK1 and PLK1 ${ }^{27}$ also

114 begs the question as to whether it has any significant role during interphase. However,

115 an important clue that condensin II can in principle function during this stage of the cell

116 cycle comes from the characterization of patients carrying a mutation of the Mcph1

117 gene, which causes a reduction in the size of the cerebral cortex known as primary

118 microcephaly (OMIM 608585) ${ }^{28,29}$. Cells from such patients have an increased

119 number of prophase-like cells ${ }^{30,31}$. The prophase-like organisation of chromosomal

120 DNA has since been shown to be mediated by condensin II 32,33 , whose abnormal

121 activity triggers premature condensation in $\mathrm{G} 2$ and late de-condensation in $\mathrm{G} 1$ 30,31,34.

$122 \mathrm{MCPH} 1$ is only found in metazoa. It contains three BRCT domains, one $\mathrm{N}$ -

123 terminal and two C-terminal, separated by a disordered region. The diverse

124 phenotypes of mutant cells have led to many different and often conflicting

125 suggestions for its roles. Binding of MCPH1's C-terminal BRCT domains to

126 phosphorylated histone $\mathrm{H} 2 \mathrm{AX}^{36}$ is thought to play a part in the DNA damage 
127 response, while the premature chromosome condensation caused by mutations within

128 its N-terminal BRCT has been attributed to this domain's defective association with

129 the SWI/SNF nucleosome remodelling complex and SET1 ${ }^{37}$. MCPH1 is also thought

130 to bind DNA and has been proposed to function in telomere maintenance, centriole

131 organisation and $\mathrm{CHK} 1$ activation ${ }^{38-42}$. In contradiction to the finding that MCPH1

132 binds directly to condensin II, it is widely believed that the $\mathrm{N}$-terminal $\sim 200$ residues of

133 MCPH1 compete with condensin II for chromosomal binding ${ }^{33}$, in other words,

$134 \mathrm{MCPH} 1$ has been proposed to occupy loci required for condensin Il's chromosomal

135 activity.

136 Given the diversity and conflicting views of MCPH1's function, we have re-

137 addressed its role in regulating chromosome structure by analysing the consequences

138 of deleting its gene in mouse ES cells. We show that loss of MCPH1 induces a

139 prophase-like organisation of chromatin during G1 and G2 but not during S phase,

140 and that this depends on condensin II. Crucially, this phenotype, which is

141 accompanied by condensin II's stable association with chromosomes, is unaffected

142 by inhibiting $\mathrm{CDK} 1$, suggesting that $\mathrm{MCPH} 1$ does not inhibit chromosome

143 condensation merely by delaying the CDK1 activation normally necessary for

144 condensin II activity as previously suggested $40,41,43$. We demonstrate that MCPH1

145 instead regulates the organisation of chromosomal DNA through the binding of

146 condensin II's NCAPG2 subunit by a conserved short linear motif (SLiM) situated

147 within its central domain. Such binding does not per se explain how MCPH1 regulates

148 condensin II and has little or no effect on its ATPase activity, at least in vitro.

149 Presumably, other domains, for example its $\mathrm{N}$-terminal BRCT, which is frequently

150 mutated in human microcephaly patients, have an effector function, by interacting with

151 other sites within the condensin II pentamer or recruit other cellular factors. 
152 A clue to the role of $\mathrm{MCPH} 1$ is the resemblance between the stable association of

153 condensin II with chromosomal DNA induced by MCPH1 ablation with the effect on

154 cohesin of mutating Wapl, which binds to the cohesin HAWK equivalent to NCAPG2,

155 namely STAG2, also using an FY SLiM. We therefore tested whether like WAPL,

$156 \mathrm{MCPH} 1$ prevents condensin II from associating stably with DNA by opening the

157 interface between NCAPH2's NTD with the neck of SMC2's ATPase domain.

158 Remarkably, a translational fusion between SMC2's C-terminus and NCAPH2's N-

159 terminus is not only functional in mouse oocytes but also resistant to the inhibition

160 mediated by an excess of MCPH1 induced by mRNA micro-injection. This raises the

161 possibility that like WAPL, MCPH1 acts by opening the interface between the kleisin's

162 NTD and the neck of the SMC ATPase domain. Finally, we investigated the

163 consequences of inducing the stable association with G2 chromosomes of both

164 cohesin and condensin and found that the chromosomal axes created by cohesin upon

165 mutating Wapl are turned into solenoids in cells deleted for both Wapl and Mcph1.

\section{RESULTS}

Mcph1 deletion induces chromosome condensation during interphase in embryonic stem cells.

171 Premature chromosome condensation is a key feature of cells isolated from patients

172 carrying Mcph1 mutations. To study this in greater detail, we used CRISPR/Cas9 to 173 delete Mcph1 in mouse E14 embryonic stem cells in which we had previously 174 introduced a Halo-tag at the C-terminal end of NCAPH2 (NCAPH2-Halo) at its 175 endogenous locus. Western blot using an anti-NCAPH2 antibody confirmed that all 176 the NCAPH2 protein present in the cell is shifted to a higher molecular weight that 
177 matches the size of the band detected by in-gel Halo-TMR fluorescence. The protein

178 expression levels are identical to the untagged protein in control cells (Figure 1A).

179 Deleting the second exon of Mcph1 induces a frameshift between exon 1 and 3 and

180 thereby complete inactivation of the gene. Western blot analysis of the targeted cells

181 confirmed the lack of any MCPH1 protein (Figure 1A).

182 Immunofluorescence microscopy revealed that Mcph1 deletion leads to a 183 substantial increase in the fraction of cells with prophase like chromosomes (Figure

184 1B). FACS analysis of cells stained with propidium iodide to measure DNA content,

185 H3PS10 specific antibodies to detect G2/M cells, and pulse labelled with EdU to

186 identify $S$ phase cells showed no overall change in cell cycle progression

187 (Supplementary figure 1). Thus, Mcph1 deletion caused little or no prophase arrest.

188 Crucially, all EdU negative cells, whether they were in G1 (H3PS10 negative) or G2

189 (H3PS10 positive), contained prophase-like chromosomes (>100 cells counted) while

190 few if any of the EdU positive S phase cells did so (Figure 1C and 3D). In wild type G1

191 and G2 cells, different centromeres and peri-centric regions cluster in chromocenters.

192 This feature is abolished in the mutant cells, where every centromere occupies an individual location (Supplementary figure 2) and each chromosome is likewise individualised into prophase-like chromatids (Figure 1B). The localisation of NCAPH2 was analysed by labelling its Halo-tag with the fluorescent TMR ligand. In wild type,

196 NCAPH2 has a diffuse nuclear distribution throughout most of the interphase, with no

197 enrichment at any particular site. The protein starts to accumulate at centromeres during G2 and subsequently along the length of chromosomes from prophase until the end of telophase. Deletion of Mcph1 caused NCAPH2 to associate with the prophaselike chromosomes in all G1 and G2 cells and become enriched at their individualised

201 centromeres (Figure 1B). 
MCPH1 restricts condensin II activity during G1 and G2.

204 The change in chromosome organisation caused by deletion of Mcph1 is accompanied by a change in the localisation of condensin II. To address whether condensin II is causing the observed phenotype, we used a Halo-PROTAC ligand to specifically induce degradation of NCAPH2-Halo (Figure 1D). Because this completely reversed the re-organisation of chromosomal DNA caused by Mcph1 deletion (Figure 1E), we conclude that altered regulation of condensin II is largely if not completely

210 responsible. Unlike the centromere dispersion, chromosome condensation, and

211 chromosome unpairing induced by loss of Slimb ubiquitin ligase or casein kinase 1 in

212 Drosophila cells, which is accompanied and caused by an increase in the level of 213 NCAPH2 ${ }^{44,45}$, deletion of Mcph1 in ES cells is accompanied by a modest but

214 nevertheless significant reduction in NCAPH2 levels (Figure 1 A \& D). The implication 215 is that MCPH1 restricts the activity of individual condensin II complexes in G1 and G2 216 cells.

MCPH1 prevents condensin II's stable association with interphase chromatin.

219 Photobleaching studies with GFP tagged condensin II subunits has revealed that they are highly mobile during interphase, suggesting that they are rarely or only transiently associated with chromatin fibres ${ }^{8}$. FRAP of TMR labelled NCAPH2-Halo confirmed this as photobleached spots of Halo-TMR fluorescence recovered $95 \%$ of their fluorescence within 1 min (Figure 2). However, deletion of Mcph1 caused a dramatic change in the dynamics, with fluorescence merely recovering to $28 \%$ of its starting level within 1 minute. After that, no further change occurred during the next 10 min

226 (Figure 2), implying that Mcph1 deletion causes $72 \%$ of condensin II complexes to 
227 associate stably with chromatin in G1 or G2 cells, thereby altering chromatin compaction during interphase.

230 The chromosome re-organisation induced by Mcph1 deletion does not depend 231 on CDK1.

232 To check whether the premature formation of prophase-like chromatids when Mcph1

233 deleted cells enter G2 might be caused by the precocious activation of CDK1, which

234 activates condensin II as wild type cells enter prophase, we compared inhibitory 235 phosphorylation of CDK1's Y15 residue and cyclin B1 localisation in wild type and 236 mutant cells. Deletion of Mcph1 neither reduced Y15 phosphorylation nor caused 237 cyclin B1 to enter nuclei prematurely (Figure 3A and B). To address this issue more 238 directly, namely whether CDK1 activity is required for condensin Il's hyperactivity, we 239 asked whether inhibition of the kinase would suppress the chromosomal re240 organisation. Treatment of both wild type and Mcph1 deleted cultures with the CDK1 241 inhibitor RO-3306 for seven hours caused most cells to accumulate in G2 with a 4C

242 DNA content (Figure 3C). The DNA organisation of wild type cells resembled that of normal G2 cells, namely no chromatid-like structures were formed and centromeres

244 were clustered in chromocenters (Figure 3D). In contrast, the chromosomal DNAs of 245 all mutant cells were organized into prophase-like chromatids with individualised 246 centromeres (Figure 3D). We conclude that the hyperactivity of condensin II in G2 247 Mcph1 deleted cells is independent of CDK1, suggesting that MCPH1 regulates condensin II directly.

250 Mcph1 deletion induces chromosomal compaction and alters inter251 chromosomal interactions 
In situ Hi-C libraries were generated for wild-type mouse E14 and Mcph1 deleted cells. In wild-type cells, chromosomal p-termini have enhanced contact frequencies with one another, as do q-termini. In contrast, p-termini are less likely to contact q-termini (Figure 4A). This is consistent with the presence of chromocenters within which ptermini co-localize with each other and likewise q-termini with each other. Because mouse chromosomes are telocentric, with centromeres located at their p-termini, the $\mathrm{HiC}$ maps confirm that centromeres co-localize with one another as do telomeres.

260 (resp., q-termini) is lost in the absence of MCPH1 (Figure 4B and C), consistent with

261 the disappearance of chromocenters as observed by microscopy. Deletion of Mcph1

262 also results in an enhancement in the frequency of long-range, intra-chromosomal contacts (Figure 4D, E and F) and enhances the frequency of inter-compartment ( $A$ to

B) contacts as compared to intra-compartment contacts ( $A$ to $A$ and $B$ to $B$ )

(Supplementary figure $3 \mathrm{~A}$ and $\mathrm{B}$ ). This finding is consistent with the compaction of individual chromosomes upon loss of MCPH1.

The HiC maps did not reveal loci moving from one compartment to the other nor any major changes in loops or contact domains (Supplementary figure 3C).

\section{Recombinant MCPH1 forms a stable complex with condensin II.}

271 Our results suggest that MCPH1 directly represses condensin II activity, possibly via

272 a direct interaction. Previous in vitro work suggested that MCPH1 binds condensin II

273 via two interfaces. One interface between the N-terminal 195 residues of MCPH1 and

274 NCAPD3 and a second binding site between a highly conserved central domain of MCPH1 (381-435) and NCAPG2 (Figure 5A and B) ${ }^{33}$. To confirm a direct interaction

276 between MCPH1 and condensin II, full length human MCPH1 and condensin II were 
expressed in insect cells and separately purified. While full-length MCPH1 was largely insoluble, we purified sufficient strep-tagged full-length MCPH1 to confirm that it could pull-down pentameric condensin II complex (Figure 5C). To localise the binding site, we expressed and purified N-terminal His-MBP-tagged truncations of MCPH1 in $E$. coli: MBP-MCPH1 1-435, MBP-MCPH1 1-195, MBP-MCPH1 196-435 and MBP-MCPH1 $348-469$

282 (Figure 5A). We found that strep-tagged condensin II could only pull-down MCPH1 constructs that included the central domain (Figure 5D). MCPH1 binding was specific (Supplementary figure 4A).

We then tested whether tetrameric condensin II, lacking either NCAPD3 or NCAPG2, could bind MCPH1 $1-435$ using pull-down assays. To exclude the MBP tag interfering with the interaction at the $\mathrm{N}$-terminus of $\mathrm{MCPH} 1$, we moved the tag to the

C-terminus. We found that removing NCAPG2 greatly reduced MCPH1 1-435 MBP pulldown, while removing NCAPD3 had no effect (Figure 5E), suggesting that the binding was mediated by the central domain of MCPH1 to NCAPG2. Further analysis with analytical size exclusion chromatography demonstrated that MBP-MCPH1 $196-435$ and condensin II co-eluted in one peak, separated from the void volume, suggesting they form a stable, soluble complex (Supplementary figure 4B).

\section{MCPH1 binds condensin II via a short linear motif.}

297 To address which part of MCPH1's central domain is necessary for binding condensin

298 II, we analysed the sequence of MCPH1 using the ConSurf server 46,47 to identify conserved sequences. Between residues 381-435 of human MCPH1, the $410-424$

300 interval stands out as a highly conserved patch within a region that is otherwise poorly 301 conserved (Figure 5B and Supplementary figure 4C). Despite its conservation, the 
sequences are predicted to be disordered, suggesting that it could be a short linear motif (SLiM) that binds to condensin II. To test this, we performed fluorescence polarisation binding assays with a 5-FAM labelled MCPH1 peptide spanning residues 407-424 and found that it bound to condensin II, with a fit $\mathrm{Kd}$ of $0.64 \pm 0.12 \mu \mathrm{M}$ (mean \pm SEM) (Figure 5F). As expected, no binding was detected to a tetrameric version of condensin II lacking NCAPG2.

SLiMs are frequently regulated by post-translation modification ${ }^{48}$ and proteomic analysis of mitotic cells previously found that $\mathrm{MCPH} 1$ can be phosphorylated within the central motif at S417 ${ }^{49}$, with S417/P418 forming a potential

311 CDK consensus site ${ }^{50}$. We therefore used a fluorescence polarisation competition 312 assay to test the effect of phosphorylating S417. In these assays, the concentration of condensin II and 5-FAM-MCPH1 ${ }_{407-424}$ is fixed and unlabelled peptides of either wild-

314 type or $\mathbf{S 4 1 7}$ phosphorylated $\mathrm{MCPH} 1_{407-424}$ are added at increasing concentrations. While the wild-type $\mathrm{MCPH} 1_{407-424}$ readily competed with 5-FAM-MCPH1407-424,

316 resulting in a fit competition $\mathrm{K}_{\mathrm{D}}$ of $5.3 \pm 1.0 \mu \mathrm{M}$, phosphorylation at $\mathrm{S} 417$ reduced the 317 affinity $\sim 10$ fold to $53 \pm 8 \mu \mathrm{M}$ (Figure $5 \mathrm{G}$ ). This suggests that CDK1 phosphorylation 318 of MCPH1 may reduce its interaction with condensin II, an effect that might have an important role in initiating chromosome condensation during prophase.

MCPH1 central domain is essential for its interaction with condensin II and its regulation in vivo.

323 To address whether this central motif is necessary for binding and regulating 324 condensin II in vivo, we created an E14 cell line in which both copies of the Ncaph2 325 gene is tagged at its C-terminus with GFP. Western blotting revealed MCPH1 in 326 immunoprecipitates generated using antibodies against GFP, and only in GFP-tagged 
327 cells expressing wild-type MCPH1 (Figure 6A). Because the MCPH1-specific antibody 328 was raised against the central domain, we used a cell line in which both copies of

329 Mcph1 and Ncaph2 were tagged with GFP and Halo respectively to test the role of the

330 central domain motif and then created a variant (Mcph1 $\left.1^{\triangle C e n G F P / \triangle C e n G F P}\right)$ lacking 15

331 residues containing the motif ( $\mathrm{S}_{400}$ SYEDYFSPDNLKER $\left.{ }_{414}\right)$. Western blotting

332 confirmed that Mcph $1^{\text {GFP/GFP }}$ cells and Mcph1 $1^{\triangle C e n G F P / \triangle C e n G F P}$ were expressed at similar

333 levels (Figure $6 \mathrm{C}$ ). The slightly increased mobility of Mcph1 $1^{\triangle C e n G F P / \triangle C e n G F P}$ and its

334 failure to be detected by the MCPH1-specific antibody confirmed deletion of the central

335 domain motif (Figure 6B). Because TMR labelled NCAPH2-Halo was detected in GFP

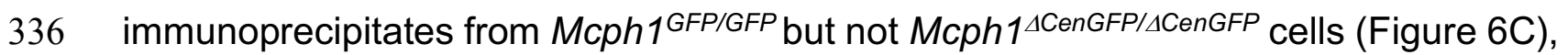

337 we conclude that MCPH1's central domain is essential for its stable interaction with

338 condensin II in vivo.

Immunofluorescence revealed that $\mathrm{MCPH} 1$ and $\mathrm{MCPH} 1^{\Delta \mathrm{Cen}}-\mathrm{GFP}$ proteins

340 show the same localisation within cells. They are both exclusively nuclear and

341 enriched in small clusters that colocalise with sites of DNA damage marked by $\gamma \mathrm{H} 2 \mathrm{aX}$

342 DNA (Figure 6D), as previously reported ${ }^{51}$. The significance of this association is

343 unclear as Mcph1 deletion has no effect on the level of $\gamma \mathrm{H} 2 \mathrm{aX}$ (Supplementary figure

344 1D). Crucially, the chromosomes of Mcph1 $1^{\triangle C e n G F P / \triangle C e n G F P}$ but not Mcph1 $1^{\text {GFP/GFP }}$ G1 and

345 G2 cells adopted the prophase-like appearance characteristic of Mcph1 deleted cells

346 (Figure 6E). Therefore, we conclude that MCPH1's central domain SLiM is essential

347 for inhibiting condensin II during interphase and inhibiting premature condensin-

348 mediated chromatin condensation.

350 MCPH1 does not alter condensin II ATPase activity or DNA binding in vitro. 
351 To address whether MCPH1 affects condensin II's activity in vitro, purified the

352 condensin II- MCPH11-435 complex using size exclusion chromatography and

353 measured its ATPase activity. The condensin II-MCPH1 1-435 complex possessed a

354 similar activity to that of condensin II alone but its stimulation by DNA was modestly

355 lower (Figure 7A). To ensure that the ATPase activity measured in these assays was

356 genuinely due to condensin II, we also purified a condensin II-MCPH1 $1_{1-435}$ complex

357 deficient in ATP binding (Q-loop mutation, SMC2 Q147L, SMC4 Q229L). As expected,

358 this mutation effectively eliminated ATPase hydrolysis (Figure 7A).

Previous work has suggested that full length MCPH1 binds to DNA and

360 chromatin ${ }^{33,42}$ so we tested whether MBP-MCPH1 $1_{1-435}$ and MBP-MCPH1 $196-435$ are

361 able to bind to a $50 \mathrm{bp}$ sequence of dsDNA using electrophoretic mobility shift assay

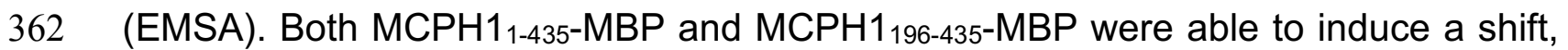
however higher concentrations of $\mathrm{MCPH} 1_{196-435} \mathrm{MBP}$ were required for a complete shift in the free DNA band, suggesting MCPH11-195 MBP could have a role in DNA binding (Figure 7B). We then examined if MCPH1 affected condensin II DNA binding, by performing condensin II EMSAs in the presence or absence of MCPH11-435-MBP.

367 The distinct $\mathrm{MCPH} 1_{1-435-\mathrm{MBP}}$ shifted band disappeared with increasing concentrations of condensin II, and there was an upward shift in the condensin II bands in the presence of $\mathrm{MCPH} 1_{1-435}-\mathrm{MBP}$ relative to the MBP condensin II control,

370 suggesting MCPH1 was binding with condensin II (Figure 7C). We then performed condensin II EMSAs in the presence or absence of $1 \mu \mathrm{M} 5-\mathrm{FAM}-\mathrm{MCPH} 1_{407-424}$ peptide. 5-FAM signal was present with condensin II shifted DNA band demonstrating that the MCPH1 peptide was sufficient to mediate comigration of MCPH1 with condensin II

374 (Figure 7D). Additionally, the presence of the 5-FAM-MCPH1 $407-424$ peptide did not 375 affect condensin II DNA binding. Collectively, this indicates that in vitro, using purified 
376 proteins, condensin II can bind MCPH1 and DNA simultaneously. Finally, these results

377 suggest that the inhibitory effect of $\mathrm{MCPH} 1$ on condensin II loading observed in vivo

378 involves a feature absent from the in vitro DNA binding assay.

380 MCPH1 overexpression inhibits the loading of condensin II on mouse meiotic

\section{1 chromosomes.}

382 Our results show that MCPH1 plays a crucial role in chromosome organisation during interphase by inhibiting condensin Il's activity in mitotic cells. We next extended our analysis of $\mathrm{MCPH} 1$ to meiotic cells by testing the effect of increased $\mathrm{MCPH} 1$ expression during the first meiotic division of mouse oocytes. To image the chromosomes, oocytes were injected with in vitro transcribed mRNA coding for $\mathrm{H} 2 \mathrm{~B}-$ mCherry to illuminate the chromosomes in magenta (Figure 8A). As previously described ${ }^{52}$, after the germinal vesicle breakdown (GVBD), bivalent chromosomes form a ball $(3.3 \mathrm{~h})$ before congressing to a metaphase plate $(7.4 \mathrm{~h})$. Cleavage of cohesin by separase along chromosome arms then converts each bivalent into a pair

391 of dyads that segregate highly synchronously to opposite poles of the cells during anaphase I $(10 \mathrm{~h})$, which is followed by extrusion of the first polar body (Figure 8B, control).

Co-injection with M. musculus MCPH1 mRNAs (Figure 8B, +MCPH1) had little effect on the chromosome congression until metaphase. However, it caused

396 chromosomes to unravel soon after the onset of anaphase, presumably because chromosomes are not stiff enough to resist to the pulling forces of the spindle, and this was accompanied by a catastrophic failure to disjoin the chromosome arms of dyads to opposite poles (Figure $8 \mathrm{~B},+\mathrm{MCPH} 1,16 \mathrm{~h}$ ). The lack of chromosome rigidity and 
400 the unravelling of the chromatin in response to traction by the spindle are reminiscent

401 of the phenotype caused by depletion of NCAPH2 ${ }^{52}$.

402 To address whether $\mathrm{MCPH} 1$ overexpression inhibits the association of 403 condensin II with chromosomes, we rescued mouse oocytes deleted for Ncaph2 404 (Ncaph2 $\left.{ }^{\text {Lox/Lox }}, Z P 3^{T g C r e}\right)$ by injecting an mRNA coding for NCAPH2-GFP as previously 405 described ${ }^{52}$. These oocytes were also injected with mRNA encoding MAD2 to arrest 406 them in meiosis I and H2B-mCherry to image and quantify the amount of chromosomal 407 NCAPH2-GFP (Figure 8C). This revealed that the injection of MCPH1 mRNAs greatly 408 reduced association of condensin II with chromosomes during metaphase I (Figure 8D 409 and E). To see if MCPH1 can also release condensin II previously associated with 410 chromosomes, we injected MCPH1 mRNA into meiosis I arrested oocytes. However, 411 this induced a much milder reduction within the first two hours (not shown), suggesting 412 that MCPH1 prevents the initial loading of condensin II on chromosomes but has little 413 effect on complexes already stably associated with them.

414 The N-terminal BRCT domain of MCPH1 was previously shown to have an 415 essential role in regulating chromosome condensation both in vitro and in vivo ${ }^{33}$. 416 Consistent with this, deletion of the N-terminal 200 amino acids of MCPH1 abolishes 417 its inhibitory effect in mouse oocytes (Figure 8D). It has also been claimed that the $\mathrm{N}$ 418 terminal domain of MCPH1 can on its own inhibit condensin II by competing for its 419 binding sites on chromosomal DNA ${ }^{33}$. However, we were unable to detect any impact 420 of over-expressing only the NTD of MCPH1 (not shown).

422 Fusion of SMC2 to NCAPH2 is resistant to MCPH1 inhibitory effect.

423 The effects of MCPH1 on condensin II resemble those of WAPL on cohesin, which is 424 thought to act by dissociating the NTD of its kleisin from the neck of SMC3's 
425 ATPase domain ${ }^{18,19,53}$. A key finding in this regard is that the fusion of the C-terminus

426 of SMC3 to the N-terminus of SCC1 causes cohesin to resist WAPL. To address

427 whether fusion of this nature has similar effect on condensin II, we created a cDNA

428 encoding a protein in which the C-terminus of SMC2 and the N-terminus of NCAPH2

429 are connected by a 57 amino acid linker containing three TEV protease cleavage sites.

430 A GFP tag was introduced at the C-terminal end of NCAPH2 to image the protein

431 (Figure 9A). Importantly, mRNAs encoding this fusion fully rescued the meiosis I

432 chromosome segregation defects of oocytes deleted for Ncaph2. Furthermore, GFP

433 fluorescence associated with the fusion protein was detected along the chromosome

434 axes of bivalent chromosomes, a distribution that is similar if not identical to that of

435 wild-type NCAPH2. Remarkably, co-injection of MCPH1 mRNAs had no adverse

436 effect on this activity, unlike controls in which MCPH1 mRNAs were co-injected with

437 NCAPH2-GFP mRNAs (Figure 9B and C). Likewise, MCPH1 prevented association of

438 NCAPH2-GFP with chromosomes but not that of the SMC2-NCAPH2-GFP fusion

439 (Figure 9C).

440 A caveat to this experiment is that the resistance to MCPH1 of the SMC2-

441 NCAPH2-GFP fusion could be due to the linker sequences associated with the N-and

442 C-termini of NCAPH2 and SMC2 respectively rather than their stable inter-connection

443 per se. If the latter were the case, cleavage of the linker using TEV protease should

444 restore sensitivity to $\mathrm{MCPH} 1$. We therefore repeated the rescue experiment but, in this

445 case co-injected mRNA encoding TEV protease (Figure 9D). Quantification of the

446 chromosomal GFP fluorescence showed that the fusion's resistance to MCPH1

447 activity is abolished by the TEV (Figure 9E and F), which is consistent with the notion

448 that resistance arises from connecting the interface between SMC2 and NCAPH2. 
Mcph1 deletion induces the coiling of cohesin vermicelli.

451 Our results suggest that condensin II's association with chromosomal DNA might be

452 regulated by $\mathrm{MCPH} 1$ through a mechanism that resembles that of cohesin by WAPL.

453 By inducing cohesin's dissociation from chromatin, albeit only rarely approximately

454 every 15 min., WAPL merely moderates the processivity of loop extrusion mediated

455 by cohesin. MCPH1 has a more drastic effect, preventing most condensin II

456 complexes from ever associating stably with chromatin. The entire architecture of

457 interphase chromatin therefore depends on these two key regulatory factors. Though

458 condensin II is presumed like cohesin to act as a DNA loop extruder, the deregulation

459 of cohesin and condensin II induces different chromosomal morphologies. Wapl

460 deletion enables cohesin to form thread-like structures and to accumulate along their

461 longitudinal axes, creating so called vermicelli. In contrast, Mcph1 deletion enables condensin II to produce soft spherical or "gumball" chromosomes and to associate stably throughout chromosomal DNA, albeit at high levels at centromeres. Strangely, condensin II does not form or accumulate along the sort of axes observed when wild type cells enter mitosis. Thus, the activities of condensin II and cohesin unleashed by Mcph1 and Wapl deletion respectively produce DNA loops with very different arrangements. We therefore set out to address two questions. First, is this difference intrinsic to differences in the behaviour of cohesin and condensin II or merely due to

469 differences in the type of cell used, namely mouse fibroblasts and ES cells? Assuming 470 that it is in fact, the former, what happens when both factors are deregulated 471 simultaneously? To this end, we altered the Mcph1 and Wapl genes in E14 cells in 472 which SCC1 is tagged with Halo and NCAPH2 with GFP. Because Wap/ deletion is 473 lethal, we generated a tamoxifen-inducible deletion allele, which enabled us to 474 compare chromosomal DNA morphology as well as localisation of SCC1-Halo and 
475 NCAPH2-GFP in four different conditions: Wild type, $\Delta W a p l, \Delta M c p h 1$ and the double 476 mutation $\Delta$ Wapl, $\Delta M c p h 1$ (Supplementary figure 5).

In wild-type cells, SCC1-Halo accumulates throughout nuclei and their 478 genomes during interphase and apart from centromeres, is largely removed from chromosomes through the action of WAPL in M phase. Condensin Il's distribution resembles that of cohesin throughout most of interphase (Figure 2 and ${ }^{8}$ ). Condensin

481 II accumulates around centromeres during G2 and along the chromatid axes created 482 through its activity during prophase (Figure 10, WT).

As previously reported for fibroblasts, Wapl deletion in E14 cells causes cohesin to create chromatid-like structures, especially during $\mathrm{G} 2$, and to accumulate along their longitudinal axes ${ }^{2}$. Because the majority of SCC1 remains on chromosomes during mitosis, most is cleaved by separase during anaphase and daughter cells inherit considerably less cohesin than normal. As a consequence, axial cohesin vermicelli are rarely if ever observed during $\mathrm{G} 1$, especially as this cell cycle phase is very short in ES cells. Pronounced vermicelli are only observed in G2. As expected, Wap/ deletion neither alters condensin Il's distribution during interphase nor hinders its accumulation along chromatid axes during mitosis. Despite cohesin's persistence on mitotic chromosomes and the participation of a large fraction in sister chromatid cohesion, condensin II still manages to localise to and help create the axes of individual chromatids, between which run inter-chromatid axes coated in cohesin

(Figure $10 \mathrm{~A}$ and $\mathrm{B}, \Delta W a p /)$. There are presumably two pools of chromosomal cohesin in post-replicative Wap/ deleted cells, one involved in cohesion and a second engaged in the loop extrusion responsible for the formation of vermicelli. The former clearly persists and accumulates along the inter-chromatid axis when loop extrusion mediated by condensin I and II individualise chromatids, but the fate of the latter is unclear. 
500 Deletion of Mcph1 had little effect on cohesin's distribution. Despite the

501 formation of gumball chromosomes during G1 and G2, cohesin remains uniformly

502 associated with chromatin and does not form vermicelli. As in wild type cells, most

503 dissociates from chromosome arms when cells enter mitosis and little can be detected

504 along the inter-chromatid axes connecting the two condensin II axes of individualised 505 chromatids (Figure 10, $\Delta$ Mcph1).

506 Deletion of both Wapl and Mcph1 had a dramatic effect. The cohesin vermicelli

507 caused by the lack of WAPL in G2 cells adopt a coiled configuration upon the

508 simultaneous deletion of Mcph1. This coiling increases during prophase. By the time

509 cells reach metaphase, the coiling leads to the formation of chromosomes that have

510 the shape of a spring (or solenoid), a configuration that is visible with SCC1-Halo,

511 NCAPH2-GFP and DNA staining (DAPI) (Figure 10, $\Delta W a p l, \Delta M c p h 1)$. Moreover, the

512 two distinct axes of condensin II associated with each chromatid remain intermingled 513 in the double mutant.

514 To analyse the axial organisation of these chromosomes in greater detail, we

515 used super-resolution three-dimensional structured illumination microscopy (3D-SIM)

516 to compare the distribution of SCC1-Halo in Wapl deleted and double mutant cells.

517 Unfortunately, fluorescence due to NCAPH2-GFP was insufficient to reveal reliable 518 images using this technique. Analysis of G2 cells revealed that a modest coiling of 519 cohesin axes surrounded by DNA loops in Wap/ single mutants is greatly accentuated

520 in double-mutant cells, with a pronounced increase in the radii of coils (Figure 11 and

521 Supplementary video 1). In metaphase cells, the cohesin axes that seem to have a 522 spring-like appearance in confocal microscopy are revealed to have a much more 523 complicated organisation, being composed of twisted segments that regularly change

524 handedness. It is noticeable that the formation of chromosomes with this morphology 
525 is not simply due to the combined activity/presence of cohesin and condensin II during

526 mitosis, which also occurs in Wapl single mutants. It only arises when both cohesin

527 and condensin were stably associated with chromatin during G2.

528 We conclude that combining the abnormal activity of condensin II unmasked by

529 deleting Mcph1 with that of cohesin unmasked by deleting Wapl leads to a major

530 transformation of chromosome structure when cells enter $\mathrm{G} 2$, that is associated with

531 coiling of the entire axis of the chromosome. Interestingly, this coiling does not have a

532 handedness that persists throughout the chromosome in metaphase. Instead, the

533 chromosome appears divided into segments whose axes are coiled with alternating

534 handedness.

535

536 DISCUSSION

537 Despite accumulation within interphase nuclei, condensin II associates with chromatin

538 only fleetingly if at all and exerts little or no effect on chromosome topology during this

539 phase of the cell cycle. It normally only associates stably with chromosomal DNA and

540 organises it into a series of loops when cells enter $M$ phase. The restriction of 541 condensin II's activity to M phase was previously attributed to its phosphorylation by

542 CDK1 $27,40,41,43$. Our finding that in the absence of MCPH1, condensin II is capable of

543 transforming the topology of chromosomal DNA in cells arrested in G2 using a CDK1

544 inhibitor implies that condensin II is capable of substantial activity in the absence of

545 CDK1-mediated phosphorylation normally associated with M phase. In other words, it

546 is MCPH1 that prevents condensin Il's association with chromosomes during G2 not

547 the lack of CDK1 phosphorylation.

548 Our observation that phosphorylation of a CDK1 consensus sequence 549 abolishes association between a conserved and essential SLiM within MCPH1's 
550 central domain and condensin Il's NCAPG2 subunit raises the possibility that CDK1 exerts at least part of its effect by preventing MCPH1's association with condensin II.

552 Our conclusion that the SLiM within MCPH1's central domain is essential for its inhibitory activity contradicts the claim that it is not necessary, an inconsistency that we attribute to the fact that previous studies tested the function of over-expressed MCPH1 alleles ${ }^{33,54}$.

Our findings as well as those of others ${ }^{30,31,35}$ show that MCPH1 is responsible

557 for inhibiting condensin II during G1 as well as G2 phase. Thus, in the absence of MCPH1, condensin II organises chromosomal DNAs into chromatid-like structures during $\mathrm{G} 1$ and $\mathrm{G} 2$ but strikingly not during $\mathrm{S}$ phase when some other (MCPH1-

560 independent) mechanism prevents it from associating stably with chromatin. MCPH1's inhibition of condensin II depends on its N-terminal BRCT domain in

562 addition to its central SLiM. Indeed, most of Mcph1 mutations identified in microcephaly patients affect the BRCT domain, which possibly interacts with some other part of condensin II once MCPH1 has been recruited via its SLiM. However, the function of this domain remains mysterious. numerous similarities between MCPH1 and WAPL, a protein that facilitates cohesin's release from chromatin ${ }^{1}$. WAPL binds to STAG, the cohesin subunit equivalent to NCAPG2, using a SLiM and its inactivation leads to cohesin's stable association with 570 chromatin $2,7,55$. Cohesin release mediated by WAPL involves dissociation of the NTD 571 of cohesin's SCC1 kleisin subunit from the coiled coil that emerges from SMC3's 572 ATPase head, known as its neck ${ }^{18,19,53}$. It is currently thought that kleisin-neck 573 dissociation takes place, albeit rarely, upon engagement of cohesin's SMC1 and 574 SMC3 ATPase heads in the presence of ATP when SMC3 is unacetylated and in the 
absence of SCC2 $18,19,56,57$. Crucially, the fusion of SMC3's C-terminus to SCC1's Nterminus completely blocks WAPL from triggering cohesin's release from chromatin 19,53. It could do so either by creating a barrier to the passage of DNA through an opened kleisin-neck interface, i.e. by blocking the exit of DNAs previously entrapped within cohesin rings, or merely by hindering kleisin-neck dissociation, which has some other poorly understood function necessary for release. chromosomes in oocytes upon over-expression of MCPH1 suggests that MCPH1 prevents condensin II's association with chromatin by a mechanism that is similar to cohesin's release by WAPL. It has been reported that engagement of SMC2 and SMC4 heads triggered by ATP binding induces the release of the NTD of the kleisin from SMC2's neck ${ }^{21,58}$, raising the possibility that MCPH1 blocks condensin Il's stable association with chromatin by facilitating such a process. Irrespective of the actual mechanics, which remains poorly understood, our observations emphasise that

590 MCPH1 blocks condensin II's association with chromosomes using a mechanism similar to that that employed by WAPL to cause cohesin release. differ in an important respect. Though WAPL alters cohesin's residence time on

594 chromatin exclusively by facilitating release, our finding that over-expression of

$595 \mathrm{MCPH} 1$ in oocytes prevents de novo association of condensin II but does not remove complexes previously associated with chromosomes raises the possibility that MCPH1 acts predominantly by aborting de novo loading.

Recent advances have shown that both human condensin and cohesin complexes extrude DNA loops ${ }^{5,59-61}$, and cryo-electron microscopy structures of yeast 
600 condensin, and yeast and human cohesin are starting to provide insight into how these

601 complexes engage with DNA $58,62-64$. In the case of cohesin, it has been suggested

602 that an early step is the clamping of DNA on top of SMC1 and SMC3 ATPase domains

603 that have engaged with each other in the presence of ATP. Clamping in this manner

604 requires cohesin's SCC2 HAWK protein, which is equivalent to condensin II's

605 NCAPD3. SCC2 is necessary to prevent cohesin's release from chromatin and may

606 perform this function by preventing dissociation of its kleisin subunit from SMC3's neck

60757,62 . If NCAPD3 had a similar role, then MCPH1 could conceivably block condensin

608 II's association with chromatin by interfering with NCAPD3's ability to block the release

609 of NCAPH2 from SMC2.

610 One of the earliest insights into the cell division cycle was that one of the main

611 constituents of the nucleus undergoes a dramatic morphological transformation shortly

612 before division, namely the transformation of an apparently amorphous mass of

613 chromatin into thread-like structures now known as chromosomes, each composed of

614 a pair of chromatids joined together. It is now recognised that this transformation is

615 brought about by condensins I and II which act by extruding DNA loops. It is also

616 recognised that interphase chromosomal DNA is not in fact amorphous but instead

617 characterised by a complex and dynamic network of interactions known as

618 topologically associated domains or TADs, which are created by cohesin that like

619 condensin is a DNA loop extruder, albeit one that is active during interphase and

620 blocked by the site specific DNA binding protein CTCF 55,65 .

621 There are two reasons why DNAs are not organised into thread-like chromatids

622 during interphase. By causing cohesin release, WAPL prevents loop extrusion by

623 cohesin going to completion while MCPH1 prevents condensin II associating with DNA

624 stably and thereby extruding loops. Crucially, inactivation of either protein leads to the 
625 formation of chromatid-like structures during interphase, albeit by different loop

626 extruders and with different actual morphologies. Interestingly, depletion of both

627 proteins simultaneously leads to a major transformation of chromosome structure as

628 cells enter mitosis, which is associated with coiling of the entire axis of the

629 chromosome. Understanding how and why this comes about through the unregulated

630 activities of cohesin and condensin II may help reveal further insight into how loop

631 extrusion creates chromosomes.

632

633 Glossary:

634 HAWK, HEAT repeat protein associated with the kleisin

635 LE, loop extrusion

636 PLC, prophase like cells

637 SLiM, short linear motif

638 TADs, topologically associating domains

639 Cryo EM, cryo electron microscopy

640 AFM, atomic force microscopy

641 GVBD, germinal vesicle break down

642

\section{Acknowledgements}

644 We thank N. Halidi and C. Monico for technical assistance, M. Inês Baptista for her

645 advice on cloning the STOP cassette, A. Szczurek for his help in fluorescence

646 quantification, S. Mahara for FACS analysis, M. Ranes and S. Guettler for the MBP

647 vector and N. Davey for SLiM conversations.

648

649 Funding 
650 This work was supported by the Wellcome Trust (Grant Ref 107935/Z/15/Z), ERC

651 grant (Proposal No 294401) and Cancer Research UK (26747). Imaging was

652 performed at the Micron Oxford Advanced Bioimaging Unit funded by a Wellcome

653 Trust Strategic Award (091911 and 107457/Z/15/Z). Funding for MSS was provided

654 by the Paul and Daisy Soros Foundation.

655

656 Author contribution

$657 \mathrm{MH}$ design, performed the experiments, wrote the manuscript and coordinated the 658 project. EC designed, performed all the in vitro biochemistry experiments and wrote 659 the manuscript. JG provided advice for the manipulation of oocytes. LS acquired and 660 analysed the SIM data. APA performed the Hi-C experiments. ELA supervised HiC 661 experiments and wrote the corresponding section. MSS and DW processed and 662 analyzed the $\mathrm{Hi}-\mathrm{C}$ data. AV supervised the project and wrote the manuscript. KN 663 supervised the project and wrote the manuscript.

664

665 Competing financial interests

666 The authors declare no competing financial interests.

667

668

669 


\section{FIGURE LEGENDS}

671 Figure 1. The deletion of Mcph1 in E14 cells induces condensin II-dependent

672 chromosome condensation in both G1 and G2 phases of the cell cycle. (A) In gel TMR-

673 Halo detection and Western blot analysis of E14 cells wild type, Ncaph2 Halo/Halo and

674 Ncaph2 $2^{\text {Halo/Halo }}$ Mcph1 $^{\text {S/A }}$. TMR signal detects NCAPH2-Halo tagged. The anti-

675 NCAPH2 antibody shows that all the NCAPH2 protein expressed is fused to the Halo-

676 tag and that the expression levels are similar to wild type but reduced after Mcph1

677 deletion. The anti-SMC2 detection shows similar levels of condensin in the three cell

678 lines. (B) Immunofluorescence analysis of Histone H3 phosphorylated on serine 10

679 (green) combined with TMR detection of NCAPH2-Halo (Red) in Mcph1wtwt and 680 Mcph $^{\Delta / \Delta}$ cells. The DNA organization was analysed using Hoechst. (C) EdU 681 incorporation in Mcph1wtwt or $M c p h 1^{\Delta / \Delta}$ cells. (D) Western blot analysis of Halo682 PROTAC induced NCAPH2-Halo degradation in wild-type, Ncaph2 Halo/Halo Mcph1wt/wt and Ncaph2 $2^{\text {Halo/Halo }} \mathrm{Mcph}^{\Delta / \Delta}$ cells using an anti-NCAPH2 antibody. Anti-SCC1 was

684 used as a loading control. (E) Immunofluorescence analysis of the chromosome 685

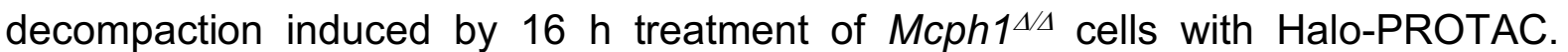
Immunofluorescence analysis of Histone H3 phosphorylated on serine 10 (green) was used to compare similar cell cycle stages. Scale bar, $5 \mu \mathrm{m}$.

Figure 2. Mcph1 deletion induces the stable binding of condensin II to the condensed chromosomes in interphase. FRAP analysis of NCAPH2-Halo turn-over on chromatin

691 in $M c p h 1^{w t / w t}(\mathbf{A})$ and $M c p h 1^{\Delta / \Delta}$ cells (B). Top row: NCAPH2-Halo signal pre-bleach, post bleach and after $70 \mathrm{sec}$ recovery. The region bleached correspond to the white

693 circle. Scale bar, $5 \mu \mathrm{m}$. (C) Quantification of the fluorescence recovery after 694 photobleaching over a 10 min post-bleach period. (Average of three experiments, total 
number of cells analysed: WT 28 cells, Mcph1 ${ }^{4 / \Delta} 30$ cells, standard deviation is

696 represented for every time point)

697

698 Figure 3. CDK1 activity is not required for the condensation phenotype induced by Mcph1 deletion. (A) Western blot analysis of CDK1 phosphorylation on tyrosine 15 in wild type cells compared to Mcph1 deleted cells. Wild-type protein extracts were treated by $\lambda$ phosphatase as a control of antibody specificity. An anti-CDK1 protein was used as a loading control. (B) Immunolocalisation of Cyclin B in Mcph1 deleted cells. (C) CDK1 activity was inhibited by incubating wild-type or Mcph1 deleted cells to RO-3306 for $7 \mathrm{~h}$. The cell cycle profile was analysed by FACS for both wild-type and Mcph1 deleted cells without treatment or after $7 \mathrm{~h}$ incubation with $9 \mu \mathrm{M}$ R0-3306. (D) All the G2 cells in Mcph1 deleted cells present the condensed phenotype after CDK1 inhibition. Scale bar, $5 \mu \mathrm{m}$.

709 Figure 4. Mcph1 deletion causes chromosome compaction and loss of chromocenters. (A) Representative subset of interactions between chromosomes 1-7

711 for wild-type and Mcph1 deletion maps (wild-type below diagonal) shows loss of

712 chromocenters in the Mcph1 deletion maps. (B) Log fold change for Mcph1 deletion

713 over wild-type for chromosomes 1-7. (C) Log fold enrichment of the Mcph1 deletion

714 map over wild-type map for the aggregated inter-chromosomal matrix. (D) Balanced

715 KR-normalized Hi-C Maps for wild-type and Mcph1 deletion maps for the

716 intrachromosomal region of chromosome 1 (wild-type below diagonal). Increased

717 interactions between distant loci in the intrachromosomal Mcph1 deletion maps is 718 seen. Color scale threshold is at the average value of each respective Hi-C map. (E) 719 Intrachromosomal contact probability for all chromosomes shows increased long- 
range interactions and diminished contact drop-off for Mcph1 deletion. (F) Average intrachromosomal contact frequency for all chromosomes shows increased longrange interactions with Mcph1 deletion.

724 Figure 5. Human condensin II interaction with MCPH1. (A) Domain structure of

$725 \mathrm{MCPH} 1$ and MBP fusion constructs that were expressed in E. coli and used in binding assays. BRCT domains are indicated in green and the central domain in yellow. (B)

Strep tag pull-down indicating full length MCPH1 binds condensin II. Full length MCPH1 and condensin II were expressed in insect cells and separately purified,

730 before being mixed on strep-tactin sepharose. Samples of input and resin after run on SDS page and visualised with silver stain. (D) Strep-tag pull-down assay indicating strep tagged condensin II pulls down MBP-MCPH1 constructs that contain the central domain, but not MBP-MCPH11-195 or MBP alone. SDS page gel visualised with Coomassie stain. (E) Strep pull-down assay showing strep-tagged pentameric condensin II or tetrameric condensin II lacking NCAPD3 can pull down MBP-MCPH1 435, while tetrameric condensin lacking NCAPG2 does not pull down MCPH1. The lower panel shows a western blot performed using strep-resin samples, blotted using an anti-NCAPD3 antibody. (F) Fluorescence polarization binding assay using 5-FAM

739 labelled $\mathrm{MCPH} 1_{407-424}$ peptide and increasing concentration of either pentameric 740 condensin or tetrameric condensin II lacking MCPH1 binding subunit NCAPG2

741 (CII $\Delta G 2)$. (G) Peptide competition assay using a fixed concentration of 5-FAM labelled

$742 \mathrm{MCPH} 1_{407-424}$ and condensin II with an increasing amount of MCPH1407-424 wild-type 743 or phosphorylated at serine 417. All error bars indicate standard deviation from three 744 replicates. 
746 Figure 6. MCPH1 interaction with condensin II is essential to prevent interphasic

747 chromosome condensation. (A) Co-immunoprecipitation of MCPH1 with NCAPH2-

748 GFP. Nuclear extracts were prepared from wild type, Ncaph2 ${ }^{\text {GFP/GFP }}$ and

749 Ncaph2 $2^{\text {GFP/GFP }}$ Mcph1 ${ }^{\Delta / \Delta}$ cells. Immunoprecipitation was performed using GFP-trap

750 agarose beads and analysed by western blot using an anti-MCPH1 antibody (IP-GFP).

$7515 \%$ of the lysate used for IP was loaded as INPUT control. Anti-Lamin B1 antibody

752 was used as a loading control. (B) Deletion of the central domain of MCPH1. To

753 address if the central domain of $\mathrm{MCPH} 1$ is necessary to mediate the interaction with

754 condensin II, we first introduced a GFP-tag at the C-terminal end of MCPH1 in

$755 N$ Naph2 $2^{\text {Halo/Halo }}$ cells as the antibody against the protein was raised against the central

756 domain. Then a second targeting was done to delete the central domain. As a result,

757 the western blot represented in panel B using anti-MCPH1 antibody detects the wild-

758 type protein or the GFP-tagged protein, homozygous Mcph1 ${ }^{\text {GFP/GFP }}$ but does not detect anything after deletion of the central domain. Using anti-GFP antibody reveals that the protein deleted for the central domain is present in the cell at similar levels as the wildtype GFP-tagged protein. A slight decrease in size is observed due to the deletion of the central domain. Anti-Lamin B1 antibody was used as a loading control. (C) Coimmunoprecipitation of NCAPH2-Halo with MCPH1-GFP. Nuclear extracts were prepared from

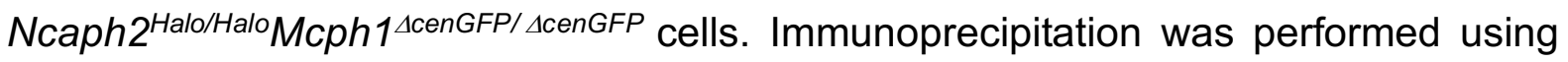
GFP-trap agarose beads and analysed by in-gel detection of NCAPH2-Halo using the

767 Halo-ligand TMR or by western blot using an anti-NCAPD3 antibody (IP-GFP). 5\% of 768 the lysate used for IP was loaded as input control. Anti-Lamin B1 antibody was used as a loading control. (D,E) Immunofluorescence analysis of the chromatin organisation 


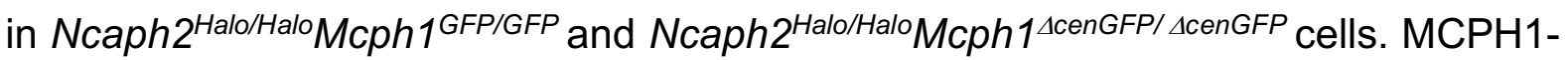

771 GFP is only detected in the cell nucleus enriched in dots colocalising with some $\gamma \mathrm{H} 2 \mathrm{AX}$

772 foci (D). The deletion of its central domain induces a similar condensation of interphasic chromosomes as the one observed after the complete loss of function of $\operatorname{Mcph} 1(\mathrm{E})$.

Figure 7. MCPH1 has little effect on condensin II ATP hydrolysis and DNA binding.

(A) ATPase rate of condensin II complex in the presence of MCPH1. Q refers to condensin II with an ATPase deficient mutation in the Q-loop. Below is an SDS page gel of the completed reaction. Error bars indicate standard deviation from three repeats. (B) EMSA assay of MBP, MCPH1 ${ }_{1-435}-\mathrm{MBP}$ and $-\mathrm{MCPH} 1_{195-435-\mathrm{MBP}}$ using 50 bp of Cy5 labelled dsDNA. (C) EMSA assay of condensin II in the presence of MBP

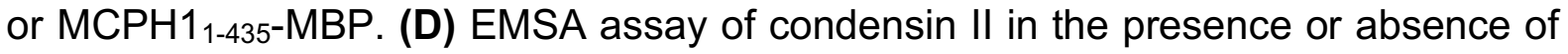
5FAM-MCPH1 peptide. Top image detecting Cy5 and bottom imaged detecting 5FAM.

Figure 8. MCPH1 prevents the association of condensin II with chromosomes. (A) Cartoon summarizing the experimental procedure corresponding to panel B. (B) Wildtype mouse oocytes were injected at the GV stage with in vitro transcribed mRNA coding for H2B-mCherry alone to mark the chromosomes in magenta (Control) or in combination with MCPH1 (+MCPH1). Meiosis I progression was followed by live cell confocal imaging. The segregation defects observed in the presence of MCPH1 are indicated by a yellow arrowhead. Maximum intensity z projection images of the main

792 time points are shown between $3.3 \mathrm{~h}$ post GVBD onwards (number of oocytes analysed in three independent experiments: control :12; +MCPH1: 19). (C) Cartoon 
795 Ncaph2 ${ }^{f / f} \mathrm{Tg}(Z P 3 C r e)$ females were injected at the GV stage with mRNA coding for

796 H2B-mCherry, MAD2 and NCAPH2-GFP only (control) or in combination with MCPH1

797 (+MCPH1) or MCPH1 deleted of the first N-terminal 200 amino acids (+MCPH1 $\Delta 200)$.

798 Oocytes were arrested $16 \mathrm{~h}$ after GVBD in metaphase I owing to MAD2

799 overexpression, and maximum-intensity z projection images of chromosomes were 800 acquired by live cell confocal imaging (Total number of oocytes analysed in three

801 experiments: control: 37, +MCPH1: 58, +MCPH1 $\Delta 200$ : 12). (E) Quantification of

802 NCAPH2-GFP signal on the chromosomes. (Number of oocytes analysed in two 803 independent experiments: control: 11; +MCPH1: 14). Scale bar, $5 \mu \mathrm{m}$

805 Figure 9. The closure of the SMC2-NCAPH2 interface prevents MCPH1 inhibitory 806 effect. (A) Schematic representation of the protein fusion between SMC2 C-terminus 807 and the N-terminus of NCAPH2 using a linker comprising three TEV protease 808 cleavage sites. (B) Cartoon summarizing the experimental procedure corresponding 809 to panel C. (C) Oocytes from Ncaph2 ${ }^{f / f} \mathrm{Tg}(\mathrm{ZP} 3 \mathrm{Cre})$ females were injected at the GV 810 stage with mRNA coding for H2B-mCherry and MCPH1 in combination with NCAPH2811 GFP (NCAPH2-GFP+MCPH1) or with the fusion (Fusion-GFP+MCPH1). Meiosis I 812 progression was followed by live cell confocal imaging. Maximum intensity z813 projections images of the time points corresponding to anaphase I when segregation 814 defects are observed (total number of oocytes analysed in three experiments:

815 NCAPH2-GFP+MCPH1: 17, Fusion-GFP+MCPH1: 18). (D) Cartoon summarizing the 816 experimental procedure corresponding to panel E. (E) Oocytes from Ncaph2/f $817 \mathrm{Tg}(\mathrm{ZP} 3 \mathrm{Cre})$ females were injected at the GV stage with mRNA coding for H2B818 mCherry, MAD2 and Fusion-GFP only or in combination with TEV protease. Oocytes 819 were arrested $16 \mathrm{~h}$ after GVBD in metaphase I owing to MAD2 over-expression and 
maximum-intensity z-projection images of chromosomes were acquired by live cell confocal imaging. (F) Quantification of Fusion-GFP signal on the chromosomes (Total number of oocytes analysed in three experiments: Fusion: 9; Fusion + MCPH1: 14, Fusion + TEV: 13, Fusion + TEV + MCPH1: 18). Scale bar, $5 \mu \mathrm{m}$.

Figure 10. Mcph1 deletion induces the coiling of the vermicelli. (A)

826 Immunofluorescence analysis of the chromatin organisation in the four conditions:

827 wild-type, $\Delta W a p l, \Delta M c p h 1$ and $\Delta W a p l \Delta M c p h 1$. In order to compare cells that are in 828 G2, prophase or metaphase, Histone H3-serine 10 (cyan) was used as a cell cycle marker. The localization of SCC1-Halo was analysed using Halo-JFX554, NCAPH2GFP using nanobodies and DNA was detected using DAPI. (B) Magnified view of cells marked with a white star in panel A. Scale bar, $5 \mu \mathrm{m}$.

Figure 11. Super-resolution 3D-SIM analysis of G2 and metaphase cells deleted for

834 Wapl or both Mcph1 and Wapl. (A) Cells deleted for $\Delta$ Wapl or $\Delta$ Wapl+ $\Delta$ Mcph1 in G2 835 or metaphase (M) were analysed by 3D-SIM. Maximum intensity projections of 16 consecutive mid sections covering $2 \mu \mathrm{m}$ in depth. The left panel shows DNA coloured in magenta and SCC1-Halo in green. The right panel shows the SCC1 signal with z-depth

838 colour-coded. Scale bar, $5 \mu \mathrm{m}$ (inset, $1 \mu \mathrm{m}$ ) (B) Representative SCC1-Halo solenoid 839 structure corresponding to one chromosome from a $\Delta W a p /+\Delta M c p h 1$ cell in $\mathrm{G} 2$ was 840 segmented (green) and overlaid to the DNA (magenta). Scale bar, $2 \mu \mathrm{m}$. (C) 3D surface rendering of the segmented and isolated solenoid from panel B. View from the top, right, left and bottom of one segmented solenoid. Scale bar, $1 \mu \mathrm{m}$. 


\section{SUPPLEMENTARY FIGURE LEGENDS}

846

847 Supplementary figure 1. The cell cycle parameters are unchanged in Mcph1 deleted

848 cells. FACS analysis of the cell cycle parameters of Mcph1 deleted cells compared to 849 wild type: (A) Analysis of the DNA content using propidium lodide (repeated twice).

850 (B) EdU incorporation (repeated twice). (C) H3 Phosphorylation on serine 10

851 (repeated twice). (D) Western blot analysis of the amount of $\gamma \mathrm{H} 2 \mathrm{AX}$ and $\mathrm{H} 3$ 852 phosphorylation on serine 10 in wild-type cells compared to Mcph1 deleted cells. Scale 853 bar, $5 \mu \mathrm{m}$.

855 Supplementary figure 2. Chromocenters disruption in Mcph1 deleted cells. 856 Immunofluorescence analysis of centromere clustering using CREST antibody 857 showing that in Mcph1 deleted cells, the centromeres are scattered in the nucleus 858 even in replicating, EdU-positive cells.

860 Supplementary figure 3. Mcph1 deletion decreases intra-compartment strength and does not affect looping. (A) Pearson correlation map at 250 kB for wildtype and Mcph1

862 deletion maps for the intrachromosomal region of chromosome 11 (wildtype below

863 diagonal). (B) Pearson correlation map at $250 \mathrm{kB}$ for wildtype and Mcph1 deletion 864 maps for the intrachromosomal region of chromosome 11 sorted by values of the principal eigenvector (wildtype below diagonal). Intra-compartmental interactions (A$A$ and $B-B)$ vs inter-compartment interactions $(A-B)$ show a relative decrease in the

867 Mcph1 deletion maps. (C) Aggregate peak analysis using the loop lists for both 868 wildtype and mcph1 deletion on both Hi-C maps shows no significant global change 869 to chromatin looping, which is also verified by direct observation of the maps. 
871 Supplementary figure 4. Condensin I does not interact with MCPH1. (A) Strep-tag

872 pull-down assay indicating strep tagged condensin I does not pull-down any MBP-

$873 \mathrm{MCPH} 1$ construct. SDS page visualised with Coomassie stain for input samples and

874 silver stain for resin samples. (B) Condensin II and MBP-MCPH1 $1_{196-435}$ form a stable

875 complex and co-elute in size exclusion chromatography. Elution profiles of condensin

876 II + MBP-MCPH1 196-435, condensin II alone and MBP-MCPH1 ${ }_{196-435}$ alone are shown

877 in green, blue and red respectively. Fractions from void, condensin II and MCPH1

878 peak were run on an SDS page and stained with Coomassie. (C) Sequence

879 conservation map of $\mathrm{MCPH}_{1-435}$ generated using the ConSurf server 46,47 , with the

880 central motif indicated. Colours range from non-conserved (green) to conserved

881 (purple), 'b' indicates residues predicted to be buried and ' $e$ ' indicates residues

882 predicted to be solvent-exposed.

883

884 Supplementary figure 5. Western blot analysis of the four conditions analysed in

885 Figures 10 and 11. The first cell line is: Ncaph2 ${ }^{\text {GFP/GFP }}$ Scc1 $1^{\text {Halo/Halo }}$ Wap/TevLox/4

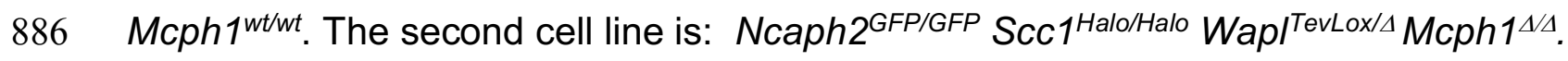

887 Wapl can be deleted in both cell lines after Tamoxifen treatment giving four 
MATERIAL AND METHODS

891 Mouse strain, in vitro culture and oocytes micro-injection.

892 Ncaph2 ${ }^{\mathrm{tm} 1 \mathrm{a}(\mathrm{EUCOMM}) W t s i}(\mathrm{MBCH}$;EPD0070-2-G090) were obtained from the Wellcome

893 Trust Sanger Institute. The corresponding flox allele was obtained as previously 894 described ${ }^{52}$. The fusion was obtained by cloning in frame Smc2 cDNA, a linker 895 containing three $\quad$ TEV cleavage sites 896 (GGGGSGGGSGGGGTGSENLYFQGPRENLYFQGGSENLYFQGTRGGGGSGGG 897 GSGGGG), Ncaph2 cDNA (Origene, MC200537) and the eGFP ORF in the pUC19 898 vector.

899 Fully grown prophase-arrested GV oocytes were isolated and injected with mRNAs 900 (5-10 pl) diluted in RNase-free water at the following concentrations: H2B-mCherry:

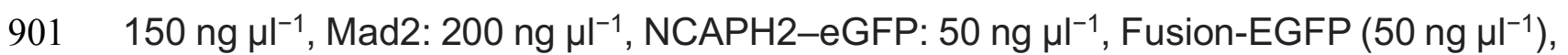
$902 \mathrm{MCPH} 1\left(200 \mathrm{ng} \mathrm{Il}^{-1}\right)$, TEV protease: $250 \mathrm{ng} \mathrm{\mu l}^{-1}$. All experimental procedures were 903 approved by the University of Oxford ethical review committee and licensed by the

904 Home Office under the Animal (Scientific procedures) Act 1986. No statistical method 905 was used to predetermine sample size. The experiments were not randomised and 906 the investigators were not blinded to allocation during experiments and outcome 907 assessment.

908 Live cell confocal imaging.

909 Oocyte live cell imaging was done in 4-well Labtek chambers (ref. 155383) in $4 \mu$ l

910 drops of M16 medium covered with mineral oil (Sigma) in a $5 \% \mathrm{CO}_{2}$ environmental

911 microscope incubator at $37^{\circ} \mathrm{C}$ (Pecon). Images were acquired using an LSM-780

912 confocal microscope (Zeiss) using the ZEN 2011 software. Between 7 and 12 slices

913 (between 1 and $4 \mu \mathrm{m}$ ) were acquired every 5 to 15 min for each stage position using 
914 the autofocus tracking macro developed in J. Ellenberg's laboratory at EMBL. For

915 detection of eGFP and mCherry, 488-nm and 561-nm excitation wavelengths and

916 MBS 488/561 filters were used. Images were further analysed using Volocity software.

917 For high-resolution videos, the lens used was a C-Apochromat $\times 63 / 1.20$ W Corr UV-

918 VIS-IR. The fluorescence was quantified using Fiji.

\section{E14 mouse embryonic stem cells culture}

920 E14 mouse embryonic stem cells were grown in Dulbecco's modified Eagle's medium

921 (DMEM; Life Technologies) supplemented with 10\% foetal calf serum (Seralab),

9222 mM L-glutamine (Life Technologies), 1x non-essential amino acids (Life

923 Technologies), $50 \mu \mathrm{M} \quad \beta$-mercaptoethanol (Life Technologies), $1 \mathrm{X}$ penicillin-

924 streptomycin solution (Life Technologies) and leukaemia inhibitory factor (LIF) made

925 in-house. All E14 cells were grown in feeder-free conditions on gelatinised plates at

$92637{ }^{\circ} \mathrm{C}$ in a humid atmosphere with $5 \% \mathrm{CO}_{2}$.

927 For conditional deletion of Wapl, Cre recombinase was induced by treating the cells 928 with $800 \mathrm{nM}$ 4-hydroxytamoxifen (OHT) for the indicated time. The degradation of

929 NCAPH2-HALO was triggered by adding HaloTag® PROTAC Ligand (Promega) at $9301 \mu \mathrm{M}$ for 16 hours.

931 The Halo Ligands (Halo-TMR Ligand, Promega, Ref G8251) were added in the culture

932 medium for 20 min at $100 \mathrm{nM}$. Cells were washed and left in the incubator with fresh

933 medium for an extra 30 min to remove the unbound ligand before being analysed by

934 immunofluorescence or western blot.

935

936 Genomic engineering using CRISPR Homology-directed repair 
937 The sgRNAs were designed using the CRISPOR online tool

938 (http://crispor.tefor.net/crispor.py) and cloned in the pSptCas9(BB)-2A-Puro(PX459)-

939 V2.0 vector (Addgene \#62988). The sgRNA cloning was done according to the

940 protocol from ${ }^{66}$.

941 To generate the targeting constructs, 1 kb homology arms were amplified by PCR (Q5-

942 NEB) from E14 cells genomic DNA and cloned in pUC19 vector using Gibson

943 Assembly Master Mix kit (New England Biolabs). The targeting construct was

944 designed such that the guide RNA sequence used for the specific targeting was

945 interrupted by the tag or contained silent mutations.

946 For each targeting, a $6 \mathrm{~cm}$ dish of E14 cells $50 \%$ confluent was transfected using $2 \mu \mathrm{g}$

947 of pX459-Cas9-sgRNA and $5 \mu \mathrm{g}$ of targeting construct using Lipofectamine 2000

948 (ThermoFisher) according to manufacturer's guidelines. The next day, cells were

949 trypsinised and plated at three different densities in $20 \mathrm{~cm}$ dishes in medium

950 supplemented with puromycin $(1 \mu \mathrm{g} / \mathrm{ml})$. The selection medium was removed $48 \mathrm{~h}$

951 later and cells were grown for approximately ten days. 96 Individual clones were then

952 picked in 96 well plates, grown for $48 \mathrm{~h}$ and split into two 96 well plates. The next day,

953 genomic DNA was prepared using $50 \mu$ of Lysis buffer (10 mM Tris HCl pH8, $1 \mathrm{mM}$

954 EDTA, $25 \mathrm{mM} \mathrm{NaCl}, 200 \mu \mathrm{g} / \mathrm{ml}$ Proteinase $\mathrm{K}$ ), incubated at $65^{\circ} \mathrm{C}$ for $1 \mathrm{~h}, 95^{\circ} \mathrm{C}$ for 10

955 min to inactivate Proteinase K. The clones were then screened by PCR (Q5-NEB) and 956 amplified to be further analysed by western blot.

957

\section{Conditional Wapl deletion}

959 To generate the TEV protease conditional cells, a series of four tandem STOP 960 cassette (Addgene, pBS.DAT-LoxStop, Jacks Lab) flanked by two LoxP sites was 961 cloned between the pCAG promoter and the PK tagged TEV protease cDNA. The 
962 CRE-ERT2 cDNA was cloned upstream under the transcriptional control of the

963 Rosa26 Splice acceptor. This construct was then flanked by $1 \mathrm{~kb}$ homology arms to

964 target the construct at the Rosa26 locus using CRISPR-HDR. After selecting and 965 amplifying of the targeted E14 clones, the TEV protease could be detected by western 966 blot $8 \mathrm{~h}$ post hydroxytamoxifen induction. Immunofluorescence analysis revealed a

967 homogeneous expression of TEV protease in all the cells.

968 In the selected clones, three TEV protease cleavage sites were targeted in Wapl

969 coding sequence after Proline 499 using CRISPR-HDR. After selecting of the targeted

970 clones, it appeared that TEV cleavage of the Wapl protein led to a new steady-state

971 in the cells in which a small amount of full-length protein was present preventing the

972 formation of vermicelli. To avoid this compensation effect, we targeted loxP sites in

973 the Wapl gene on both sides of exon 4 to induce the deletion of the gene

974 simultaneously as the TEV cleavage of the protein already present in the cell. This

975 combined strategy induced a complete loss of function of Wap/ within 8 hours and the

976 formation of vermicelli.

977

sgRNA used for CRISPR-HDR

\begin{tabular}{|l|l|}
\hline Targeted Gene & sgRNA \\
\hline NCAPH2-GFP & GGTGGAAAGTAGTATATACC \\
\hline NCAPH2-Halo & GGTGGAAAGTAGTATATACC \\
\hline MCPH1 deletion Sg5' & GGTGTGCAATTCCTAGTGTG \\
\hline MCPH1 deletion Sg3' & AGCTGTTCCTTAGAACACGA \\
\hline MCPH1-GFP & ACAGTGAGACATCTACAATG \\
\hline STOP-TEV & CATGGATTTCTCCGGTGAAT \\
\hline WAPL-Lox-TEV Sg5' & AATGGGTGCTTATAATTAGC \\
\hline
\end{tabular}




\begin{tabular}{|l|l|}
\hline WAPL-Lox-TEV Sg3' & ACAATGTCACAATGGCTCAT \\
\hline SCC1-Halo & ATAATATGGAACCGTGGTCC \\
\hline DELCEN-MCPH1 & CGTTGAGGCTTCTTCCTATG \\
\hline Two Guide RNA used & AATGGGTGCTTATAATTAGC \\
in combination & ACAATGTCACAATGGCTCAT \\
\hline
\end{tabular}

\section{Immunofluorescence detection}

981 E14 cells were plated on glass coverslips (Marienfeld, High precision, 22×22, N¹.5H, 982 Ref 0107052) in 6-well plates. $48 \mathrm{~h}$ later, cells were labelled with the Halo Ligand 983 (Halo-JFX554, Janelia $100 \mathrm{nM} 30 \mathrm{~min}){ }^{67}$ or EdU (5 min at $\left.10 \mu \mathrm{M}\right)$, washed PBS and 984 then fixed in 3.8\% Formaldehyde (Sigma-F8775) in PBS for $15 \mathrm{~min}$. After three PBS 985 washes, cells were permeabilised in $0.5 \%$ Triton $\mathrm{X}-100$ in PBS for 10 min and then 986 washed three times in PBS. After 20 min in blocking buffer: PBS 3\% BSA (Sigma, 987 A4503). The coverslips were transferred in a wet chamber and covered with $100 \mu$ of 988 antibody solution in blocking buffer. After $1 \mathrm{~h}$ at room temperature, the coverslips were 989 washed three times in blocking solution and incubated with the secondary antibody for $9901 \mathrm{~h}$ (AlexaFluor 594 or 488, 1/500, Life Technology). After three washes in PBS, DNA 991 was labelled using Hoechst (Sigma, 33342, $5 \mu \mathrm{g} / \mathrm{ml}$ ) for $15 \mathrm{~min}$. The coverslips were 992 then washed in PBS, mounted in Vectashield (H1000) and sealed using nail varnish. 993 Imaging was done using an LSM 780 confocal microscope (Zeiss) using the ZEN 2011 994 software. EdU was detected according to the manufacturer's instruction (Click-iT EdU 995 Imaging kit, Invitrogen). CDK1 inhibition was performed by incubating the cells in E14 996 medium supplemented with $9 \mu \mathrm{M}$ RO-3306 (SIGMA SML0569) for $7 \mathrm{~h}$ before fixation. 


\section{Immunoprecipitation and western blot}

999 The cells were collected using Trypsin and washed in PBS twice. The cell pellet was

1000 resuspended in 10 vol. of buffer $\mathrm{A}$ (10 mM HEPES pH7.9, $1.5 \mathrm{mM} \mathrm{MgCl}$, $10 \mathrm{mM} \mathrm{KCl,}$

$10011 \mathrm{mM}$ DTT, 1 mM PMSF, 1x complete protease inhibitor (Roche, 04693132001) and

1002 incubated $15 \mathrm{~min}$ on ice. After centrifugation at $4^{\circ} \mathrm{C}$ at $2000 \mathrm{rpm}$ for $5 \mathrm{~min}$ to remove

1003 the supernatant, the pellet was resuspended in 3 vol. of buffer A + NP40 $(0.1 \%)$ and

1004 incubated for $15 \mathrm{~min}$ on ice followed by another centrifugation at $4{ }^{\circ} \mathrm{C} 4000 \mathrm{rpm}$ for

$10055 \mathrm{~min}$ to remove the supernatant and resuspended in lysis buffer: (20 mM Tris pH7.5,

$1006200 \mathrm{mM} \mathrm{NaCl}, 1 \%$ Triton X-100, 0.1\% sodium deoxycholate, 1 mM DTT, 1 mM PMSF,

1007 1x complete protease inhibitor (Roche, 04693132001), 1x super nuclease). The

1008 suspension was passed through a 26GA needle 10 times, left on ice for $1 \mathrm{~h}$ and

1009 centrifuged at $20000 \mathrm{~g}$ for $30 \mathrm{~min}$. Proteins were quantified by Bradford (KitBiorad

1010 5000006, spectrophotometer BIOCHROM). Each IP was performed using $1 \mathrm{mg}$ of

1011 protein in $1 \mathrm{ml}$ in lysis buffer. GFP-Trap agarose beads (GTA-10, Chromotek) were

1012 washed in lysis buffer and incubated according to the supplier's instructions with the

1013 protein extract overnight at $4{ }^{\circ} \mathrm{C}$. Washed five times in Lysis buffer and separated by

1014 SDS-PAGE, imaged using Fujifilm FLA-7000 imager if Halo ligand was used and then

1015 transferred overnight on Nitrocellulose membrane. After Ponceau red evaluation of

1016 the transfer quality, the membrane was blocked in PBS-0,1\%Tween $20+5 \%$ non-fatty

1017 milk for an hour and incubated with the antibody overnight in PBS-0,1\%Tween $20+$

$10185 \%$ non-fatty milk. Western blots were analysed using LI-COR Odyssey Fc imager.

1019

1020 Antibodies list

1021 NCAPH2: Rabbit polyclonal produced on demand by Eurogentec (WB:1/1000).

1022 SCC1: Millipore, 53A303, mouse monoclonal antibody (WB:1/1000). 
1023 SMC2: Cell Signaling Technology, D23C5, rabbit monoclonal Antibody (WB:1/500).

1024 Lamin B1: Abcam Ab133741, rabbit monoclonal (WB:1/1000).

1025 MCPH1: Cell Signaling Technology, D38G5, rabbit monoclonal Antibody (WB:1/1000).

1026 CREST: Immunovision HCT0-100, human autoantibody (IF: 1/500)

1027 H3PS10: Millipore, clone 3H10, mouse monoclonal (WB:, IF:1/2000).

$1028 \gamma \mathrm{H} 2 \mathrm{aX}$ : Millipore, clone JBW301, mouse monoclonal (WB:, IF:1/500).

1029 CDK1: Cell Signaling Technology, cdc2, 77055, rabbit polyclonal (WB:1/1000).

1030 Phospho-CDK1: Cell Signaling Technology, phospho-cdc2 (Tyr15) antibody, 9111, 1031 rabbit polyclonal (WB:1/1000).

1032 WAPL: provided by J.M. Peters's Lab (WB:1/1000).

1033 GFP: Abcam, ab290, rabbit polyclonal (WB: 1/1000, IF:1/500).

1034 PK-Tag: Biorad, MCA 1360G, mouse monoclonal (WB:1/1000).

1035 Cyclin B1: Cell Signaling Technology, 4138T, rabbit monoclonal (IF:1/200).

1036 NCAPD3: Bethyl Laboratory, A300-604A-M, rabbit polyclonal (WB:1/1000).

1037

1038 Halo in gel imaging

1039 Cells were incubated with Halo-TMR ligand (100 nM) for 30 min then washed in ligand1040 free medium and analysed by immunofluorescence or for protein purification. After 1041 SDS-PAGE, the fluorescence was measured in the gel using an Imager Fujifilm FLA10427000.

1045 Live-cell imaging was performed in a spinning disk confocal system (PerkinElmer 1046 UltraVIEW) with an EMCCD (Hamamatsu) mounted on an Olympus IX8 microscope 1047 with Olympus 60x 1.4 N.A. and 100x 1.35 N.A. objectives. Image acquisition and 
quantitation were performed using Volocity software. During imaging, cells were maintained at $37^{\circ} \mathrm{C}$ and $5 \% \mathrm{CO} 2$ in a humidified chamber. FRAP was carried out with a $488 \mathrm{~nm}$ laser beam, 100\% power, 15-30 ms. The fluorescence intensity

1051 measurement was performed by using ImageJ. All signals were subjected to

1052 background correction. The fluorescence intensity of unbleached and bleached areas was normalized to that of initial pre-bleaching images using the EasyFRAP website.

1054

\section{In-situ Hi-C}

$1056 \mathrm{Hi}-\mathrm{C}$ libraries were generated as described in ${ }^{68}$, analysed using the Juicer pipeline ${ }^{69}$ 1057 and visualised with Juicebox ${ }^{70}$. We sequenced $785,454,085 \mathrm{Hi}-\mathrm{C}$ read pairs in wild1058 type mouse ES cells, yielding 509,278,039 Hi-C contacts; we also sequenced 1,814,815,287 Hi-C read pairs in Mcph1 deleted cells, yielding 1,284,169,272 Hi-C contacts. Loci were assigned to A and B compartments at $100 \mathrm{kB}$ resolution. Loops were called with HiCCUPS at $25 \mathrm{kB}, 10 \mathrm{kB}$, and $5 \mathrm{kB}$ resolution. Contact domains

1062 were called at $10 \mathrm{kB}$ resolution. Contact frequency analysis was performed as 1063 described in ${ }^{71}$. All code used for these analyses is publicly available at 1064 (github.com/aidenlab), with a help forum for questions at (aidenlab.org/forum.html).

\section{Structured illumination microscopy}

1067 Super-resolution 3D-SIM was performed on a DeltaVision OMX SR system (GE

1068 Healthcare) equipped with sCMOS cameras (PCO) and 405, 488 and 568 nm lasers, 1069 using a 60x NA 1.42 PlanApo oil immersion objective (Olympus). To minimise 1070 artefacts due to spherical aberration Raw data sets were acquired with a z-distance of $125 \mathrm{~nm}$ and 15 raw images

1072 per plane (5 phases, 3 angles). Reconstructions were performed with SoftWoRx 6.2 1073 (GE Healthcare) using channel-specifically measured optical transfer functions (OTFs) 
1074 generated from $100 \mathrm{~nm}$ diameter green and red FluoSphere beads (ThermoFisher),

1075 respectively, and Wiener filter set to 0.0030. For DAPI acquisitions, the sample was

1076 excited with the $405 \mathrm{~nm}$ laser and the emission detected in the green channel and

1077 reconstructed with a green OTF. This is enabled by the broad emission spectrum of

1078 DAPI and empirically resulted in better reconstructions than reconstructing blue 1079 emission with a 'blue' OTF obtained typically less intense blue FluoSphere beads.

$1080 \quad$ All data underwent quality assessment via SIMcheck ${ }^{72}$ to determine image

1081 quality via analysis of modulation contrast to noise ratio (MCNR), spherical aberration

1082 mismatch, reconstructed Fourier plot and reconstructed intensity histogram values.

1083 Reconstructed 32-bit 3D-SIM datasets were thresholded to the stack modal intensity

1084 value and converted to 16-bit composite z-stacks to discard negative intensity values using SIMcheck's "threshold and 16-bit conversion" utility and MCNR maps were generated using the "raw data modulation contrast" tool of SIMcheck. To eliminate false positive signals from reconstructed noise, we applied SIMcheck's 'modulation

1088 contrast filter' utility. Briefly, this filter sets masks out all pixels, where the underlying

1089 MCNR value in the raw data fall below an empirically chosen threshold MCNR value 1090 of 6.0 , followed by a Gaussian filter with 0.8 pixel radius (xy) to smoothen hard edges 109173.

1092 Colour channels were registered in 3D with the open-source software

1093 Chromagnon $0.85^{74}$ determining alignment parameter (x,y,z-translation, $\mathrm{x}, \mathrm{y}, \mathrm{z}-$ 1094 magnification, and z-rotation) from a 3D-SIM dataset acquired on the date of image acquisition of multicolour-detected 5-ethenyl-2'-deoxyuridine (EdU) pulse replication labelled C127 mouse cells serving as biological 3D alignment calibration sample ${ }^{75}$. 
1099 Cells were incubated with EdU $(10 \mu \mathrm{M})$ for 5 min, collected using trypsin, washed with

1100 PBS and fixed in 3.8\% Formaldehyde (Sigma-F8775) in PBS for 15 min. After three

1101 PBS washes, cells were permeabilised in $0.5 \%$ Triton $\mathrm{X}-100$ in PBS for 10 min and

1102 then washed three times in PBS. Cells were then incubated for 20 min in blocking

1103 buffer: PBS 3\% BSA (Sigma, A4503), incubated for $1 \mathrm{~h}$ with H3PS10 antibody

1104 (1/1000), washed three times in PBS and then incubated with fluorescent secondary

1105 antibody (Alexa Fluor 488, 1/500, Life Technology) incubated with propidium iodide

1106 (Sigma, $30 \mu \mathrm{g} / \mathrm{ml}$ ) and then analysed by FACS. 15,000 cells were analysed for each

1107 data point. EdU detection was done following the manufacturer's instruction (Click-iT

1108 EdU Imaging kit, Invitrogen).

1110 Protein purification

1111 Human condensin II pentameric and tetrameric complexes were purified as previously

1112 described ${ }^{59}$. Full length MCPH1 was cloned into the pLIB vector and viral bacmids

1113 were generated using Tn7 transposition in DH10EMBacY cells (Geneva biotech),

1114 transfected into Sf9 cells using Cellfectin II (GIBCO) and resultant virus harvested after

11153 days. Virus was further amplified in Sf9 cells before being used to infect High Five

1116 cells for protein expression. High Five cells were harvested by centrifugation 3 days

1117 after infection. Cell pellets were resuspended in purification buffer (20 mM HEPES pH

1118 8, $300 \mathrm{mM} \mathrm{KCl}, 5 \mathrm{mM} \mathrm{MgCl} 2,1 \mathrm{mM}$ DTT, 10\% glycerol) supplemented with 1 Pierce

1119 protease inhibitor EDTA-free tablet (Thermo Scientific) per $50 \mathrm{~mL}$ and $25 \mathrm{U} / \mathrm{ml}$ of

1120 Benzonase (Sigma) and lysed with a Dounce homogenizer followed by brief

1121 sonication. The lysate was cleared with centrifugation, loaded onto a StrepTrap HP

1122 (GE), washed with purification buffer and eluted with purification buffer supplemented

1123 with $5 \mathrm{mM}$ desthiobiotin (Sigma). Size exclusion chromatography was performed 
1124 using purification buffer on a Superdex 200 16/60 column (GE), and protein containing

1125 fractions separated from the void volume were pooled and concentrated.

1126 MCPH1 residue 1-435, 1-195 and 196-435 were cloned into a pET vector with an N-

1127 terminal 6xHis-MBP fusion tag or with an N-terminal 6xHis and C-terminal MBP tag,

1128 and expressed in E. coli BL21 (DE3) pLysS cells (Novagen). Cells were grown at 37

$1129{ }^{\circ} \mathrm{C}$, induced with $1 \mathrm{mM}$ IPTG for $4 \mathrm{~h}$, before being harvested by centrifugation and

1130 flash frozen. The cell pellet was resuspended in MCPH1 purification buffer (20 mM

1131 Tris $\mathrm{pH} 7.5,150 \mathrm{mM} \mathrm{NaCl}, 10 \%$ glycerol, 1 mM DTT, Pierce protease inhibitor EDTA-

1132 free tablet), lysed with sonication on ice, treated with Benzonase (Sigma-Aldrich) (10

$1133 \mu \mathrm{L}$ per $100 \mathrm{~mL}$, with $1 \mathrm{mM} \mathrm{MgCl}$ ) and cleared via centrifugation. Cleared cell lysate

1134 was filtered with a $5 \mu \mathrm{m}$ filter, imidazole was added to a final concentration of $10 \mathrm{mM}$

1135 and incubated with pre-equilibrated His-Pure NTA resin. The resin was washed with

$1136 \mathrm{MCPH} 1$ purification buffer, then washed with wash buffer $20 \mathrm{mM}$ Tris $\mathrm{pH}$ 7.5, $500 \mathrm{mM}$

$1137 \mathrm{NaCl}, 20 \mathrm{mM}$ imidazole, before elution with $20 \mathrm{mM}$ Tris $\mathrm{pH}$ 8, 300mM NaCl, $500 \mathrm{mM}$

1138 Immidazole, 10\% glycerol. Protein was diluted 2-fold with buffer TA (20 mM Tris pH 8,

$11395 \%$ glycerol, 1 mM DTT), and loaded onto a HiTrap Q Fast Flow column or loaded on

1140 to HiTrap Heparin HP column (GE) and eluted with a gradient of buffer TB (20 mM

1141 Tris $\mathrm{pH} 8,2 \mathrm{M} \mathrm{NaCl}, 5 \%$ glycerol, $1 \mathrm{mM}$ DTT). For protein used in ATPase assays,

1142 final size exclusion chromatography was performed using purification buffer and a 1143 Superdex 200 10/300 or 16/60 column (GE).

1145 Pull-downs

1146 Condensin complexes $(0.1 \mu \mathrm{M})$ were mixed with at least a 10-fold molecular excess

1147 of MBP MCPH1 constructs or MBP protein in $200 \mu \mathrm{L}$ and incubated with $40 \mu \mathrm{L}$ of 1148 Strep-tactin sepharose resin (IBA) in $\mathrm{MCPH} 1$ purification buffer. The resin was 
1149 washed 5 times, before being eluted by boiling in 1x NuPAGE LDS sample buffer with

$115050 \mathrm{mM}$ DTT. Samples of $10 \%$ input and resin elution were run on $4-12 \%$ NuPAGE Bis-

1151 Tris gels against Color Prestained Protein Standard, Broad Range (NEB) and stained

1152 with Instant Blue (Expedeon) or silver stain (Life Technology).

1153 The absence of the CAP-D3 subunit was confirmed by western blot analysis of the

1154 output protein samples. For this, the tetrameric condensin II pull-down was run on an

1155 SDS page gel and transferred to a nitrocellulose membrane (Amersham). The

1156 membrane was then blocked with 5\% milk-powder in TBS-T, before being probed with

1157 a mouse anti-CAP-D3 antibody (Santa Cruz, Sc-81597, used at 1/1000), then a goat

1158 DyLight 800 florescent anti-mouse secondary antibodies (Cell Signaling Technology,

1159 5257, used at 1/5000). The membrane was imaged using a LI-COR imager.

1160

1161 Fluorescence polarisation assays

1162 Peptides used in fluorescence polarization assays were synthesised by Genscript and

1163 are shown in Table 1. The concentration of 5FAM wild-type MCPH1407-422 was

1164 determined using the 5-FAM extinction coefficient of $83,000(\mathrm{cmM})^{-1}$ at $493 \mathrm{~nm}$. Non-

1165 labelled peptides had TFA removed to less than $1 \%$ and were accurately quantified

1166 using Genscript's amino acid analysis service. All peptides were solubilised in DMSO

1167 and diluted to a working concentration in FP assay buffer (20 mM Tris pH 7.5, $200 \mathrm{mM}$

$1168 \mathrm{NaCl}, 1 \mathrm{mM}$ DTT). Peptides in competition experiments were diluted in a two-fold

1169 series with FP assay buffer supplemented with DMSO, such that DMSO concentration

1170 was constant for all peptide concentrations.

1171

1172 Table 1: Peptides used in FP experiments

\begin{tabular}{|l|l|}
\hline Name & Sequence \\
\hline
\end{tabular}




\begin{tabular}{|c|c|}
\hline 5FAM-MCPH1 407-422 & 5FAM- CGESSYDDYFSPDNLKER \\
\hline $\mathrm{MCPH}_{407-422}$ & CGESSYDDYFSPDNLKER \\
\hline MCPH1 $407-422 p S 417$ & CGESSYDDYF $\{$ pSER\}PDNLKER \\
\hline
\end{tabular}

1174 FP binding assays were performed with $0.3 \mu \mathrm{M}$ of 5-FAM labelled wild-type MCPH1 $407-$

1175422 and $0.625,1.25,1.75,2.5,3.5$ and $5 \mu \mathrm{M}$ of pentameric condensin II or tetrameric

1176 condensin II lacking the CAPG2 subunit, in a total volume of $40 \mu$ in half-area black

1177 plates (Constar). The plate was incubated at room temperature for $20 \mathrm{~min}$, before

1178 being read with an Omega plate reader (BMG Labtech) at 5 min intervals and

1179 monitored to ensure binding had reached equilibrium. Each plate was read three

1180 times, and three replicates were performed at each protein concentration.

1181 FP competition titrations were performed as above, but with $0.63 \mu \mathrm{M}$ of condensin II,

$1182 \quad 0.3 \mu \mathrm{M}$ of $5 \mathrm{FAM}-\mathrm{MCPH} 1_{407-422}$ and indicated amount of competing peptide. Data was

1183 normalised by subtracting the FP signal for 5FAM-MCPH1 ${ }_{407-422}$ in the absence of

1184 condensin II at each peptide concentration and divided by the background-subtracted

1185 signal of sample with $0.63 \mu \mathrm{M}$ of condensin II and $0.3 \mu \mathrm{M}$ of $5 F A M-M C P H 1_{407-422}$

1186 without any competing peptide. Each plate was read 4 times and each experiment was

1187 performed a total of 3 times.

1188 Fluorescence polarisation data was fit using equations for direct binding and directly

1189 competitive binding, as presented by ${ }^{76}$.

ATPase assays

1192 Complexes of wild type or ATPase hydrolysis deficient Q-loop mutants condensin II

1193 with $\mathrm{MCPH} 1_{1-435} \mathrm{MBP}$ were purified with gel filtration on a Superose 6 10/300 column, 1194 along with wild-type only control in ATPase assay buffer (20 mM Tris pH 7.5, $150 \mathrm{mM}$ 
$1195 \mathrm{NaCl}, 1 \mathrm{mM}$ DTT). Prior to gel-filtration all samples were treated with Tev protease to

1196 remove N-terminal 6x His-tag on MCPH1 1 -435MBP. ATPase assays were performed

1197 using the EnzChek ${ }^{\mathrm{TM}}$ Phosphate Assay Kit (Invitrogen) modified for a 96 well plate

1198 format ${ }^{77} .50$ bp double stranded DNA sequence with the same as that used in the

1199 EMSA (without a fluorescent label). Reactions contained $50 \mathrm{nM}$ protein with or without

1200800 nM DNA. Final conditions included 1 mM ATP and a total salt concentration of 50

$1201 \mathrm{mM}$. Protein/DNA was preincubated in reaction mix without ATP for 15 minutes at

1202 room temperature before the reaction was started by addition of ATP immediately

1203 before putting it in the plate reader to track phosphate release. A standard curve

1204 ATPase rate was determined from a linear fit of data from the first $60 \mathrm{~min}$.

1205

1206 Electromobility shift assays

1207 EMSAs were performed using $50 \mathrm{nM}$ of 50 bp double stranded DNA labelled with Cy5 1208 at the 5' with the sequence:

1209 CTGTCACACCCTGTCACACCCTGTCACACCCTGTCACACCCTGTCACACC

1210 For MCPH1 EMSAs, MCPH1-MBP constructs were diluted in $20 \mathrm{mM}$ Tris pH 7.5, 150

$1211 \mathrm{mM} \mathrm{NaCl}, 10 \%$ glycerol, $1 \mathrm{mM}$ DTT and mixed 1 in 2 with DNA in $20 \mathrm{mM}$ HEPES pH

$12128,300 \mathrm{mM} \mathrm{KCl}, 5 \mathrm{mM} \mathrm{MgCl} 2,10 \%$ glycerol and $1 \mathrm{mM}$ DTT.

1213 For condensin II/MCPH1 EMSAs, condensin II diluted in 20 mM HEPES pH 8, 300

$1214 \mathrm{mM} \mathrm{KCl}, 5 \mathrm{mM} \mathrm{MgCl} 2,10 \%$ glycerol and $1 \mathrm{mM} \mathrm{DTT}$ and mixed with $\mathrm{MCPH}_{1-435-\mathrm{MBP}}$,

1215 MBP or 5-FAM-MCPH1 ${ }_{407-424}$ diluted in in $20 \mathrm{mM}$ Tris $\mathrm{pH} 7.5,150 \mathrm{mM} \mathrm{NaCl}, 10 \%$

1216 glycerol, $1 \mathrm{mM}$ DTT. DNA was then added to a final concentration of $50 \mathrm{nM}$. Protein

1217 and DNA were incubated on ice for $15 \mathrm{~min}$, before being loaded in a $2 \%$ agarose gel

1218 and run in 0.5x TBE buffer for $30 \mathrm{~min}$. Gel was imaged using a Typhoon. 


\section{REFERENCES}

1220 1. Yatskevich, S., Rhodes, J. \& Nasmyth, K. Organization of Chromosomal DNA by SMC Complexes. Annu. Rev. Genet. 53, 445-482 (2019).

2. Tedeschi, A. et al. Wapl is an essential regulator of chromatin structure and chromosome segregation. Nature 501, 564-568 (2013).

3. Challa, K. et al. Meiosis-specific prophase-like pathway controls cleavageindependent release of cohesin by Wapl phosphorylation. bioRxiv 1-27 (2018) doi:10.1101/250589.

4. Hill, L. et al. Wapl repression by Pax5 promotes $\mathrm{V}$ gene recombination by Igh loop extrusion. Nature 584, 142-147 (2020).

5. Davidson, I. F. et al. DNA loop extrusion by human cohesin. Science (80-. ). 366, 1338-1345 (2019).

6. Ganji, M. et al. Real-time imaging of DNA loop extrusion by condensin. Science (80-. ). 360, 102-105 (2018).

7. Wutz, G. et al. ESCO1 and CTCF enable formation of long chromatin loops by protecting cohesinstag1 from WAPL. Elife 9, 1-33 (2020).

8. Gerlich, D., Hirota, T., Koch, B., Peters, J. M. \& Ellenberg, J. Condensin I stabilizes chromosomes mechanically through a dynamic interaction in live cells. Curr. Biol. 16, 333-344 (2006).

9. Hansen, A. S., Pustova, I., Cattoglio, C., Tjian, R. \& Darzacq, X. CTCF and cohesin regulate chromatin loop stability with distinct dynamics. Elife 6, 1-33 (2017).

1241 10. Cutts, E. E. \& Vannini, A. Condensin complexes: understanding loop extrusion one conformational change at a time. Biochem. Soc. Trans. 48, 2089-2100 (2020). 
1244 11. Hirota, T., Gerlich, D., Koch, B., Ellenberg, J. \& Peters, J. M. Distinct functions of condensin I and II in mitotic chromosome assembly. J. Cell Sci. 117, 64356445 (2004).

12. Ono, T. et al. Differential contributions of condensin I and condensin II to mitotic chromosome architecture in vertebrate cells. Cell 115, 109-121 (2003).

13. Ono, T., Fang, Y., Spector, D. L. \& Hirano, T. Spatial and Temporal Regulation of Condensins I and II in Mitotic Chromosome Assembly in Human Cells. Mol. Biol. Cell 15, 3296-3308 (2004).

14. Walther, N. et al. A quantitative map of human Condensins provides new insights into mitotic chromosome architecture. J. Cell Biol. 217, 2309-2328 (2018).

15. Gibcus, J. H. et al. A pathway for mitotic chromosome formation. Science (80). 359, (2018).

16. Baxter, J. \& Aragón, L. A model for chromosome condensation based on the interplay between condensin and topoisomerase II. Trends Genet. 28, 110117 (2012).

17. Elbatsh, A. M. O. et al. Cohesin Releases DNA through Asymmetric ATPase-

18. Beckouët, F. et al. Releasing Activity Disengages Cohesin's Smc3/Scc1 Interface in a Process Blocked by Acetylation. Mol. Cell 61, 563-574 (2016).

19. Chan, K. L. et al. Cohesin's DNA exit gate is distinct from its entrance gate and is regulated by acetylation. Cell 150, 961-974 (2012). Smc3-kleisin interface. Science (80-. ). 346, 963-967 (2014).

1268 21. Hassler, M. et al. Structural Basis of an Asymmetric Condensin ATPase Cycle. 
Mol. Cell 74, 1175-1188.e9 (2019).

22. Lee, B. G. et al. Cryo-EM structures of holo condensin reveal a subunit flip-flop mechanism. Nat. Struct. Mol. Biol. 27, 743-751 (2020).

23. Buheitel, J. \& Stemmann, O. Prophase pathway-dependent removal of cohesin from human chromosomes requires opening of the Smc3-Scc1 gate. EMBO J. 32, 666-676 (2013).

24. Huis In't Veld, P. J. et al. Characterization of a DNA exit gate in the human cohesin ring. Science (80-. ). 346, 968-972 (2014).

25. Ono, T., Yamashita, D. \& Hirano, T. Condensin II initiates sister chromatid resolution during S phase. J. Cell Biol. 200, 429-441 (2013).

26. Schwarzer, W. et al. Two independent modes of chromatin organization revealed by cohesin removal. Nature 551, 51-56 (2017).

27. Abe, S. et al. The initial phase of chromosome condensation requires Cdk1mediated phosphorylation of the CAP-D3 subunit of condensin II. Genes Dev. 25, 863-874 (2011).

28. Woods, C. G., Bond, J. \& Enard, W. Autosomal recessive primary microcephaly $(\mathrm{MCPH})$ : A review of clinical, molecular, and evolutionary findings. Am. J. Hum. Genet. 76, 717-728 (2005).

29. Thornton, G. K. \& Woods, C. G. Primary microcephaly: do all roads lead to Rome? Trends Genet. 25, 501-510 (2009).

30. Neitzel, H. et al. Premature chromosome condensation in humans associated with microcephaly and mental retardation: A novel autosomal recessive condition. Am. J. Hum. Genet. 70, 1015-1022 (2002).

31. Trimborn, M. et al. Mutations in microcephalin cause aberrant regulation of chromosome condensation. Am. J. Hum. Genet. 75, 261-266 (2004). 
32. Trimborn, M., Schindler, D., Neitzel, H. \& Hirano, T. Misregulated chromosome condensation in $\mathrm{MCPH} 1$ primary microcephaly is mediated by condensin II. Cell Cycle 5, 322-326 (2006).

33. Yamashita, D. et al. MCPH1 regulates chromosome condensation and shaping as a composite modulator of condensin II. J. Cell Biol. 194, 841-854 (2011).

34. Arroyo, M. et al. MCPH1 is essential for cellular adaptation to the G2-phase decatenation checkpoint. FASEB J. 33, 8363-8374 (2019).

35. Arroyo, M. et al. MCPH1, mutated in primary microcephaly, is required for efficient chromosome alignment during mitosis. Sci. Rep. 7, 1-12 (2017).

36. Venkatesh, T. \& Suresh, P. S. Emerging roles of MCPH1: Expedition from primary microcephaly to cancer. Eur. J. Cell Biol. 93, 98-105 (2014).

37. Leung, J. W. et al. SET nuclear oncogene associates with microcephalin/MCPH1 and regulates chromosome condensation. J. Biol. Chem. 286, 21393-21400 (2011).

38. Cicconi, A. et al. Microcephalin 1/BRIT1-TRF2 interaction promotes telomere replication and repair, linking telomere dysfunction to primary microcephaly. Nat. Commun. 11, (2020).

39. Lin, S. Y. \& Elledge, S. J. Multiple tumor suppressor pathways negatively regulate telomerase. Cell 113, 881-889 (2003).

40. Alderton, G. K. et al. Regulation of mitotic entry by microcephalin and its overlap with ATR signalling. Nat. Cell Biol. 8, 725-733 (2006).

41. Gruber, R. et al. MCPH1 regulates the neuroprogenitor division mode by coupling the centrosomal cycle with mitotic entry through the Chk1-Cdc25 pathway. Nat. Cell Biol. 13, 1325-1334 (2011).

42. Chang, H. Y. et al. Microcephaly family protein MCPH1 stabilizes RAD51 
filaments. Nucleic Acids Res. 48, 9135-9146 (2020).

43. Tibelius, A. et al. Microcephalin and pericentrin regulate mitotic entry via centrosome-associated Chk1. J. Cell Biol. 185, 1149-1157 (2009).

44. Buster, D. W. et al. SCFSlimb ubiquitin ligase suppresses condensin IImediated nuclear reorganization by degrading Cap-H2. J. Cell Biol. 201, 49-63 (2013).

45. Nguyen, H. Q. et al. Drosophila Casein Kinase I Alpha Regulates Homolog Pairing and Genome Organization by Modulating Condensin II Subunit Cap-H2 Levels. PLoS Genet. 11, 1-32 (2015).

46. Berezin, C. et al. ConSeq: The identification of functionally and structurally important residues in protein sequences. Bioinformatics 20, 1322-1324 (2004).

47. Ashkenazy, H. et al. ConSurf 2016: an improved methodology to estimate and visualize evolutionary conservation in macromolecules. Nucleic Acids Res. 44, W344-W350 (2016).

48. Van Roey, K. et al. Short linear motifs: Ubiquitous and functionally diverse protein interaction modules directing cell regulation. Chem. Rev. 114, 6733-

49. Oppermann, F. S. et al. Combination of chemical genetics and phosphoproteomics for kinase signaling analysis enables confident identification of cellular downstream targets. Mol. Cell. Proteomics 11, 1-12 (2012).

50. Errico, A., Deshmukh, K., Tanaka, Y., Pozniakovsky, A. \& Hunt, T. Identification of substrates for cyclin dependent kinases. Adv. Enzyme Regul. 50, 375-399 (2010). 
1344 51. Rai, R. et al. BRIT1 regulates early DNA damage response, chromosomal integrity, and cancer. Cancer Cell 10, 145-157 (2006).

1346 52. Houlard, M. et al. Condensin confers the longitudinal rigidity of chromosomes. Nat. Cell Biol. 17, 771-781 (2015).

53. Eichinger, C. S., Kurze, A., Oliveira, R. A. \& Nasmyth, K. Disengaging the Smc3/kleisin interface releases cohesin from Drosophila chromosomes during interphase and mitosis. EMBO J. 32, 656-665 (2013).

54. Wood, J. L., Liang, Y., Li, K. \& Chen, J. Microcephalin/MCPH1 associates with the condensin II complex to function in homologous recombination repair. J. Biol. Chem. 283, 29586-29592 (2008).

55. Li, Y. et al. The structural basis for cohesin-CTCF-anchored loops. Nature 578, 472-476 (2020).

56. Chan, K. L. et al. Pds5 promotes and protects cohesin acetylation. Proc. Natl.

Acad. Sci. U. S. A. 110, 13020-13025 (2013).

57. Srinivasan, M. et al. Scc2 counteracts a wapl-independent mechanism that releases cohesin from chromosomes during G1. Elife 8, 1-34 (2019).

58. Lee, B. G. et al. Cryo-EM structures of holo condensin reveal a subunit flip-flop mechanism. Nat. Struct. Mol. Biol. 27, 743-751 (2020).

59. Kong, M. et al. Human Condensin I and II Drive Extensive ATP-Dependent Compaction of Nucleosome-Bound DNA. Mol. Cell 79, 99-114.e9 (2020).

60. Golfier, S., Quail, T., Kimura, H. \& Brugués, J. Cohesin and condensin extrude DNA loops in a cell-cycle dependent manner. Elife 9, 1-34 (2020). DNA by loop extrusion. Science (80-. ). 366, 1345-1349 (2019).

1368 62. Collier, J. E. et al. Transport of DNA within cohesin involves clamping on top of 
engaged heads by SCC2 and entrapment within the ring by SCC3. Elife 9, 136 (2020).

63. Higashi, T. L. et al. A Structure-Based Mechanism for DNA Entry into the Cohesin Ring. Mol. Cell 79, 917-933.e9 (2020).

64. Shi, Z., Gao, H., Bai, X. \& Yu, H. Cryo-EM structure of the human cohesinNIPBL-DNA complex. 1459, 1454-1459 (2020).

65. Rao, S. S. P. et al. Cohesin Loss Eliminates All Loop Domains. Cell 171, 305320.e24 (2017).

66. Ran, F. A. et al. Genome engineering using the CRISPR-Cas9 system. Nat. Protoc. 8, 2281-2308 (2013).

67. Grimm, J. B. et al. A General Method to Improve Fluorophores Using Deuterated Auxochromes. JACS Au 1, 690-696 (2021).

68. Rao, S. S. P. et al. A 3D map of the human genome at kilobase resolution reveals principles of chromatin looping. Cell 159, 1665-1680 (2014).

69. Durand, N. C. et al. Juicer Provides a One-Click System for Analyzing LoopResolution Hi-C Experiments. Cell Syst. 3, 95-98 (2016).

70. Durand, N. C. et al. Juicebox Provides a Visualization System for Hi-C Contact Maps with Unlimited Zoom. Cell Syst. 3, 99-101 (2016).

71. Sanborn, A. L. et al. Chromatin extrusion explains key features of loop and domain formation in wild-type and engineered genomes. Proc. Natl. Acad. Sci.

72. Ball, G. et al. SIMcheck: A toolbox for successful super-resolution structured illumination microscopy. Sci. Rep. 5, 1-12 (2015).

73. Rodermund, L. et al. Time-resolved structured illumination microscopy reveals key principles of Xist RNA spreading. Science (80-. ). 372, eabe7500 (2021). 
1394 74. Matsuda, A., Schermelleh, L., Hirano, Y., Haraguchi, T. \& Hiraoka, Y. Accurate and fiducial-marker-free correction for three-dimensional chromatic shift in biological fluorescence microscopy. Sci. Rep. 8, 1-3 (2018).

1397 75. Kraus, F. et al. Quantitative 3D structured illumination microscopy of nuclear structures. Nat. Protoc. 12, 1011-1028 (2017).

76. Roehrl, M. H. A., Wang, J. Y. \& Wagner, G. A general framework for development and data analysis of competitive high-throughput screens for small-molecule inhibitors of protein-protein interactions by fluorescence polarization. Biochemistry 43, 16056-16066 (2004).

1403 77. Voulgaris, M. \& Gligoris, T. G. A protocol for assaying the ATPase activity of recombinant cohesin holocomplexes. Methods Mol. Biol. 2004, 197-208 (2019). 
Figure 1
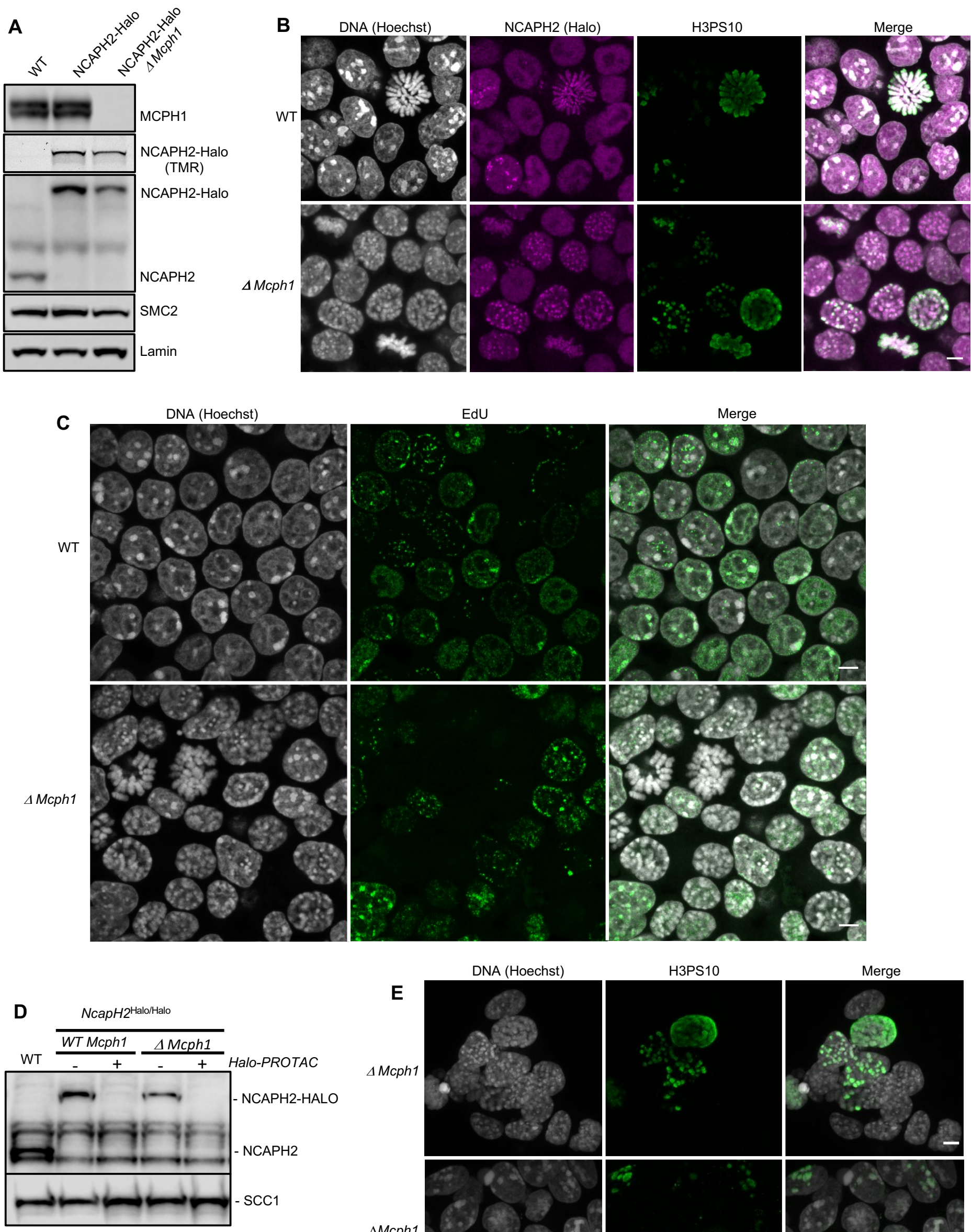

Halo-PROTAC NCAPH2-HALO

NCAPH2

$\operatorname{SCC} 1$

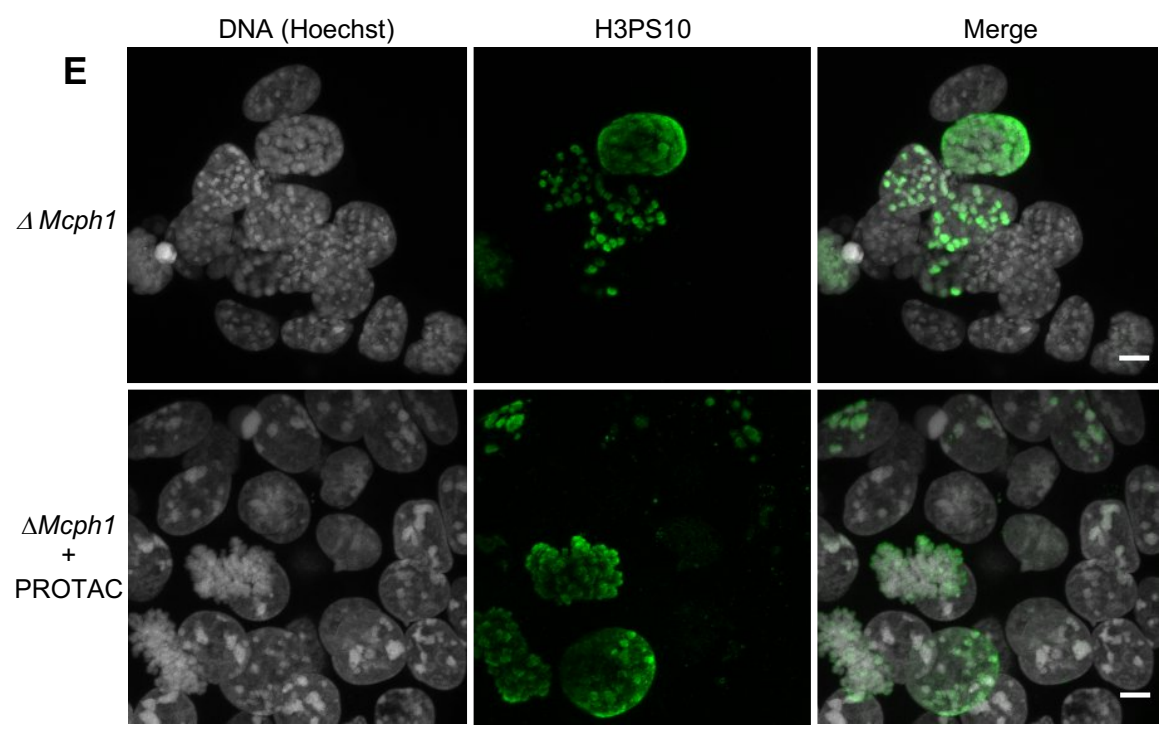




\section{Figure 2}
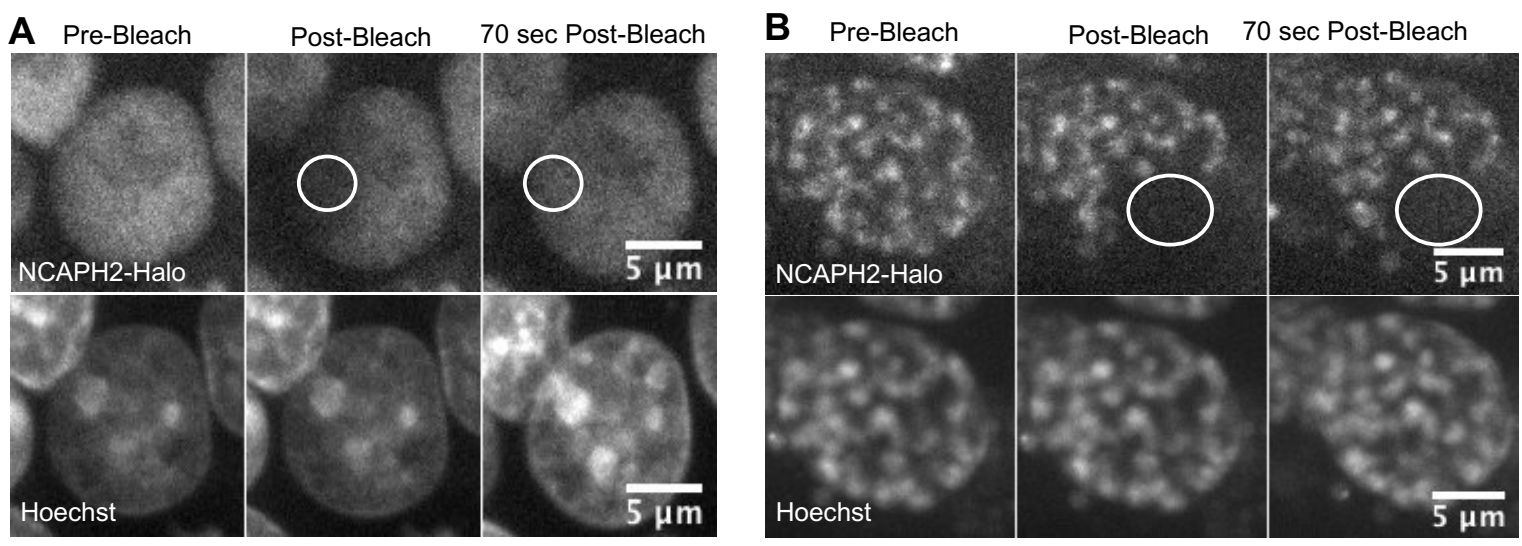

\section{C}

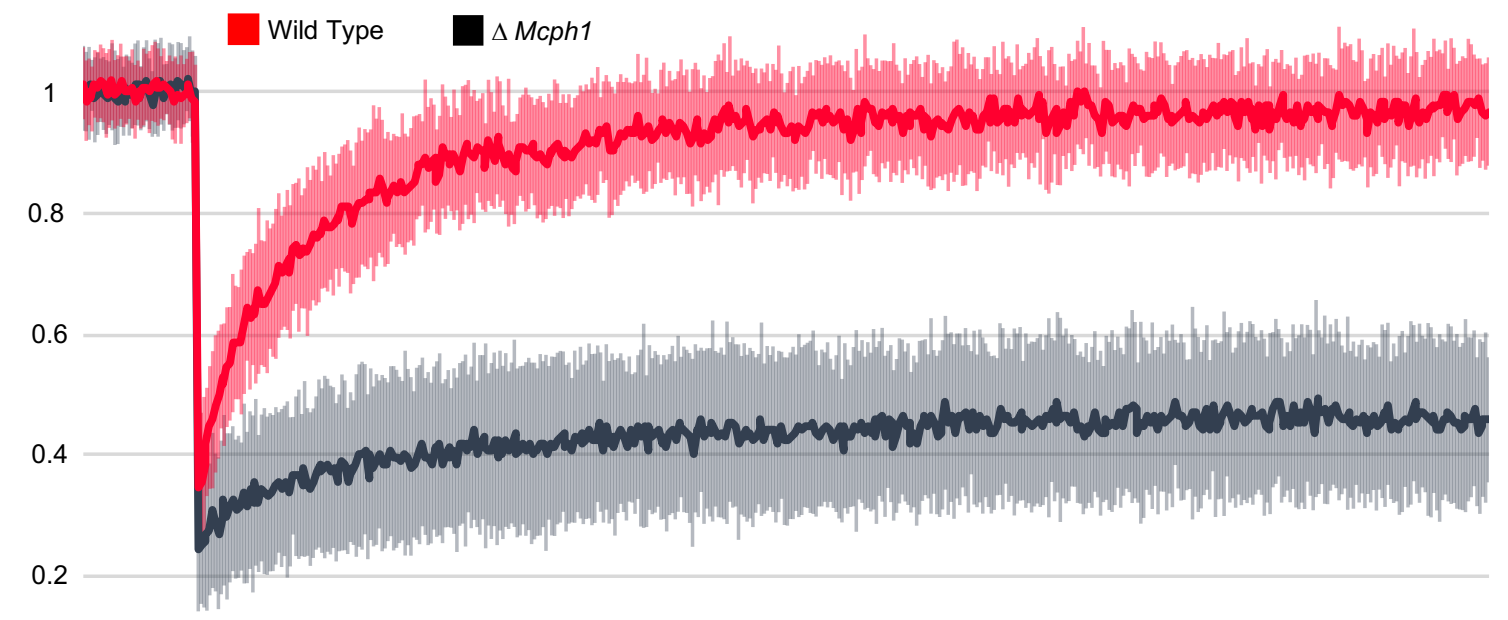

0 
Figure 3 (which was not certified by peer review) is the author/funder. All rights reserved. No reuse allowed without permission.
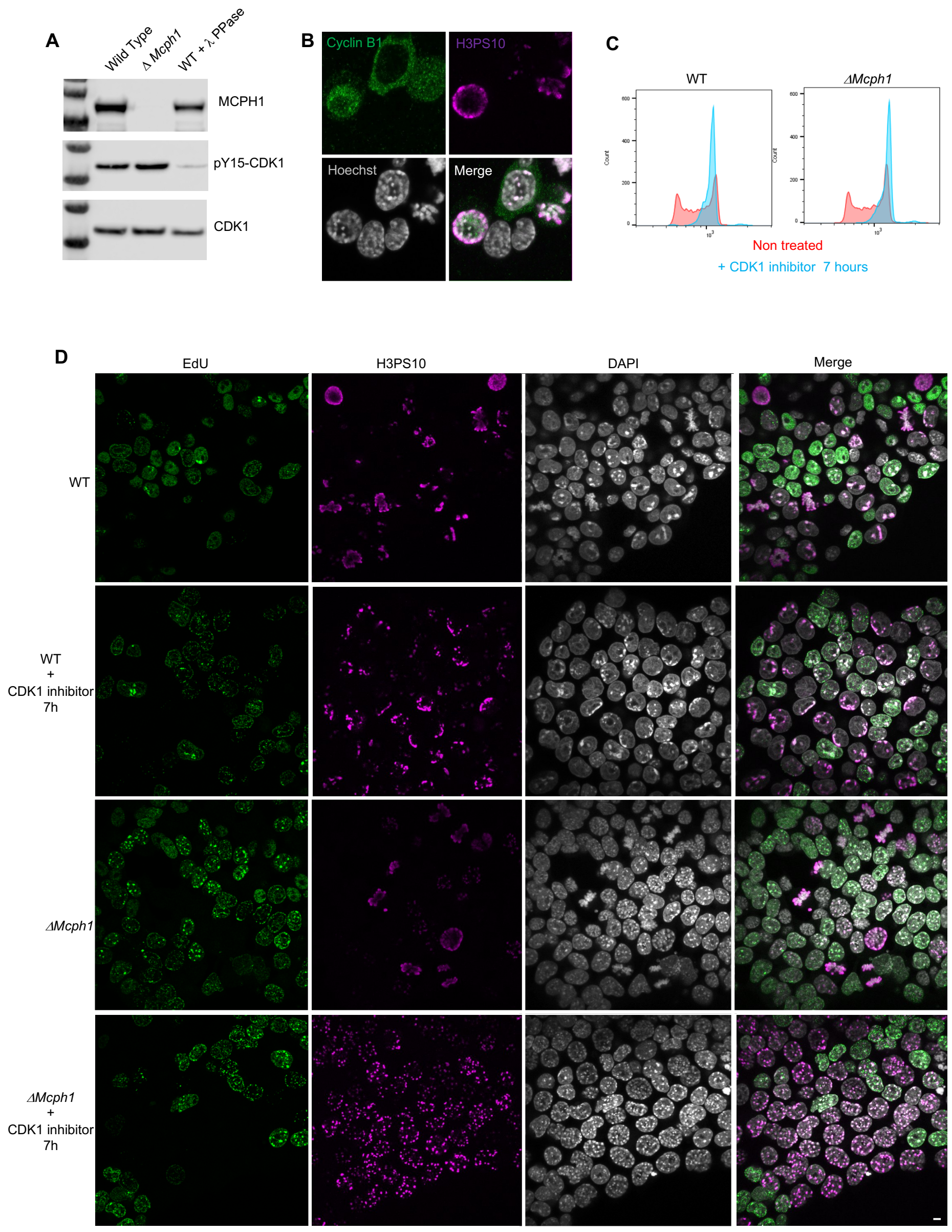
Figure 4

A

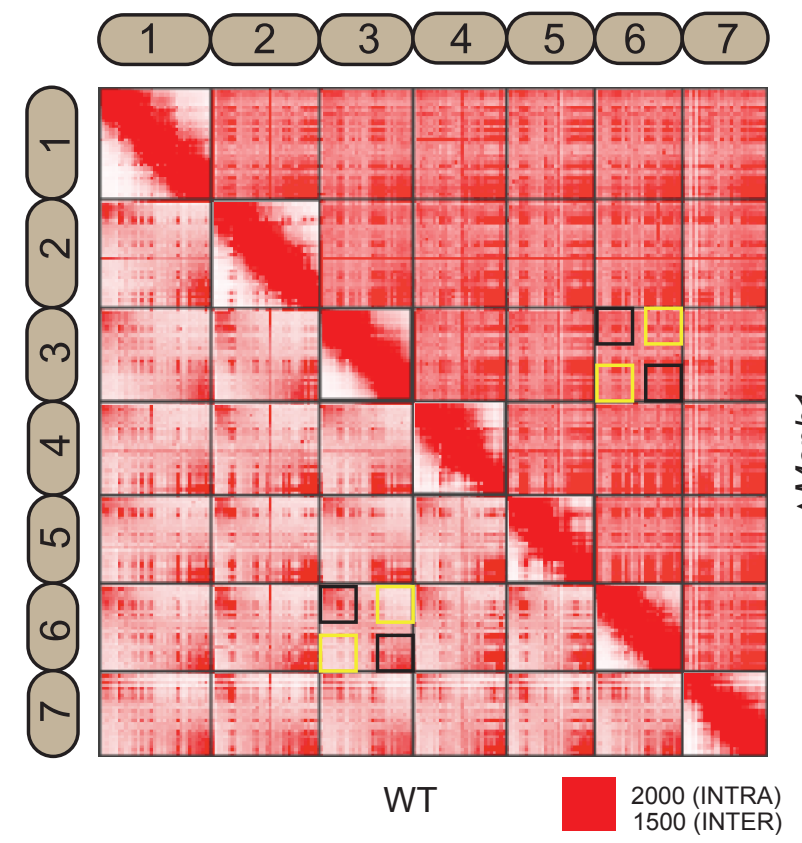

C

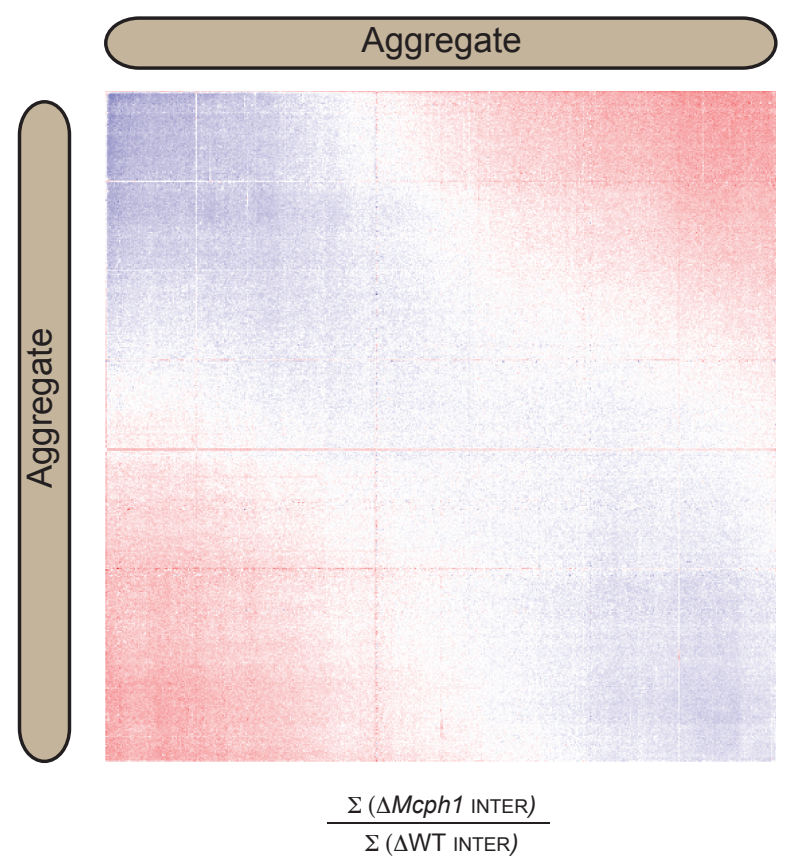

$\mathbf{E}$

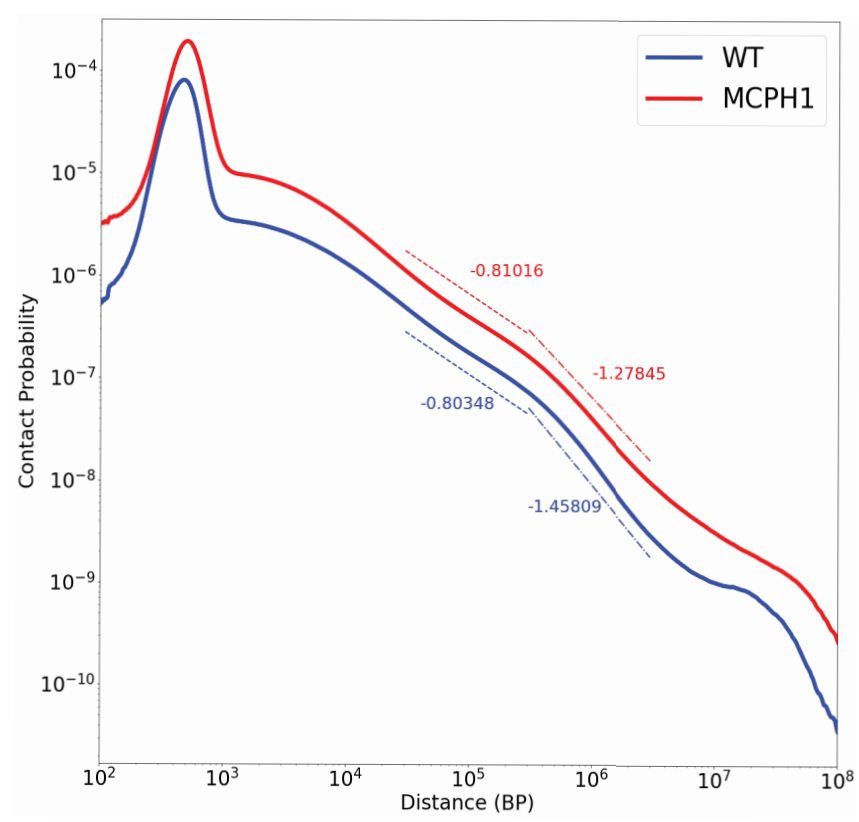

B
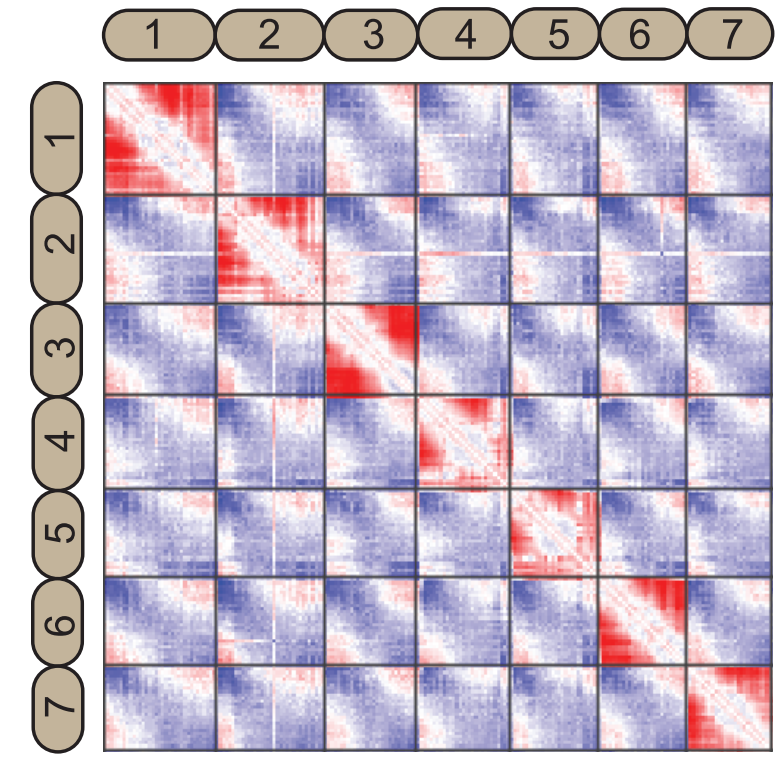

D

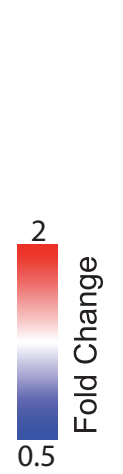

$\mathbf{F}$

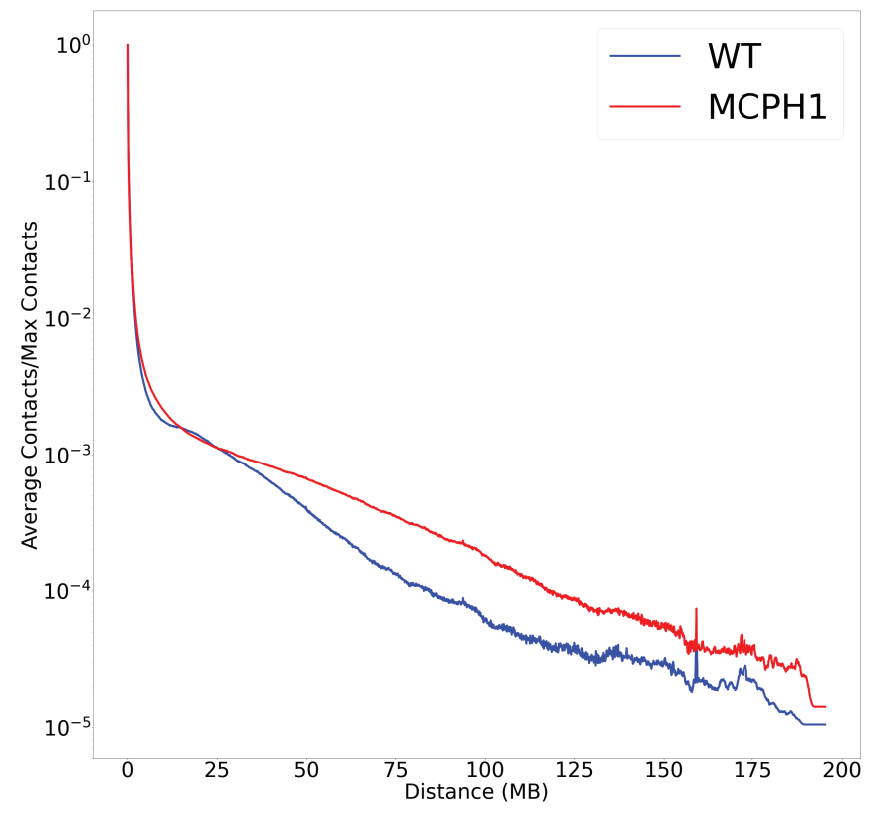




\section{Figure 5}

A

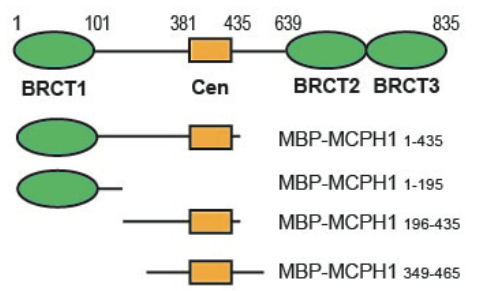

\section{D}
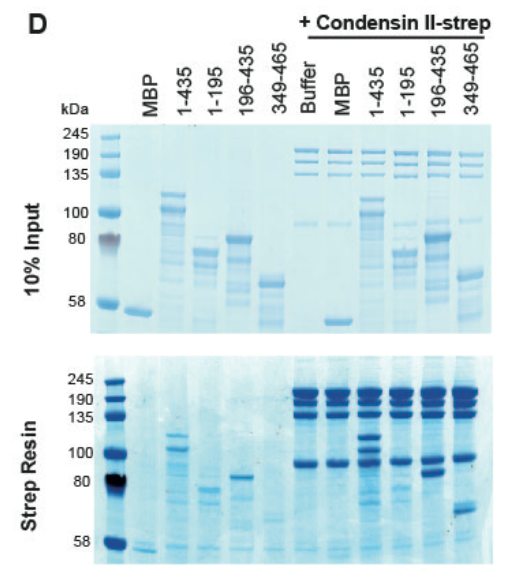

B

381 I MPRIOLCRS EDRLOHVAGP

401 A LEALSCGES SY D Y F SPDN

421 LKERYSENLP PESOL 435

The conservation scale:

? \begin{tabular}{llllll|l|l|l|l}
1 & 2 & 3 & 4 & 5 & 6 & 7 & 8 & 9
\end{tabular}

E

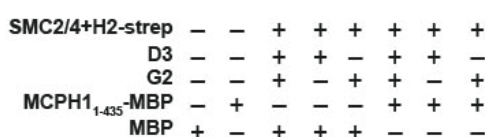

$\mathrm{kDa}$
245

${ }^{\mathrm{kDa}}{ }^{\mathrm{kD}} \mathrm{C}=--=$

产 ${ }^{190}$

すे

100
80

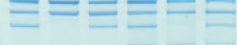

80

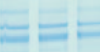

$58=---$

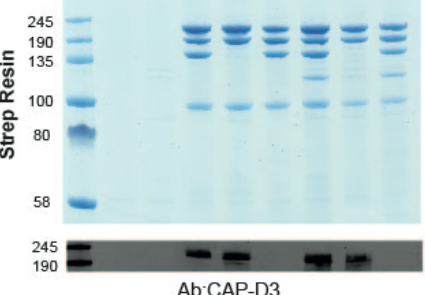

Ab:CAP-D3
C

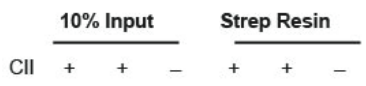

MCPH1-strep - + + -++
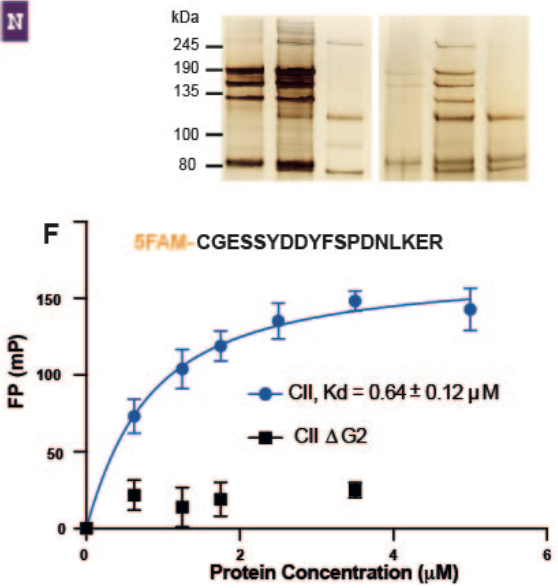

G

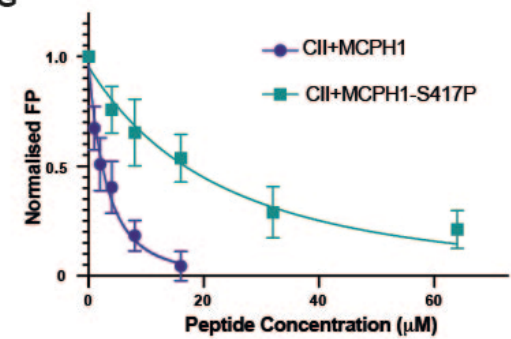


bioRxiv preprint doi: https://doi.org/10.1101/2021.07.20.453033; this version posted July 20, 2021. The copyright holder for this preprint

(which was not certified by peer review) is the author/funder. All rights reserved. No reuse allowed without permission.

Figure 6

A

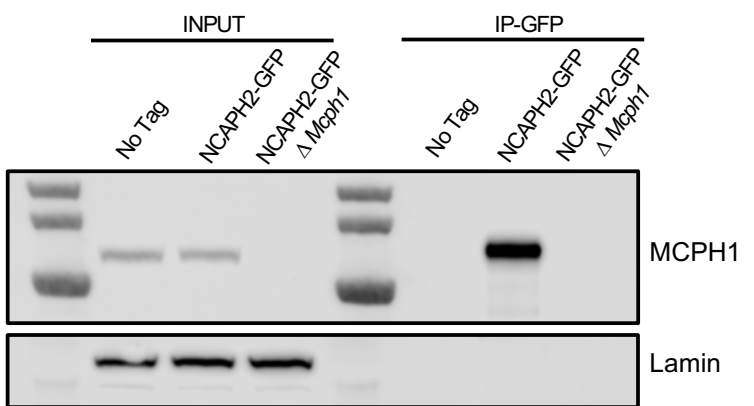

B

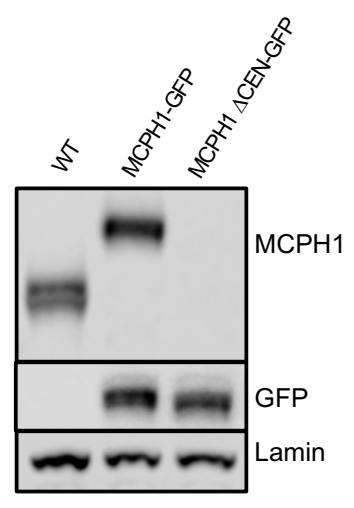

C

IP-GFP

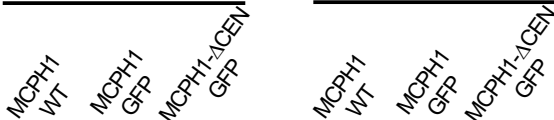

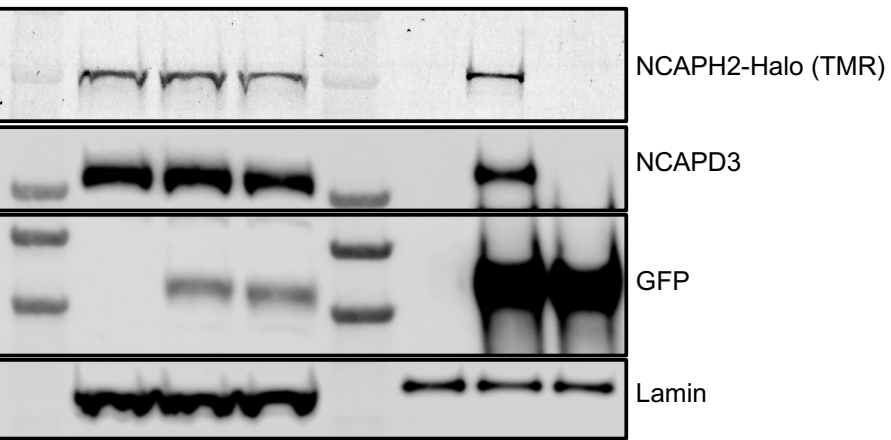

D

DNA (Hoechst)

MCPH1-GFP

$\gamma \mathrm{H} 2 \mathrm{aX}$
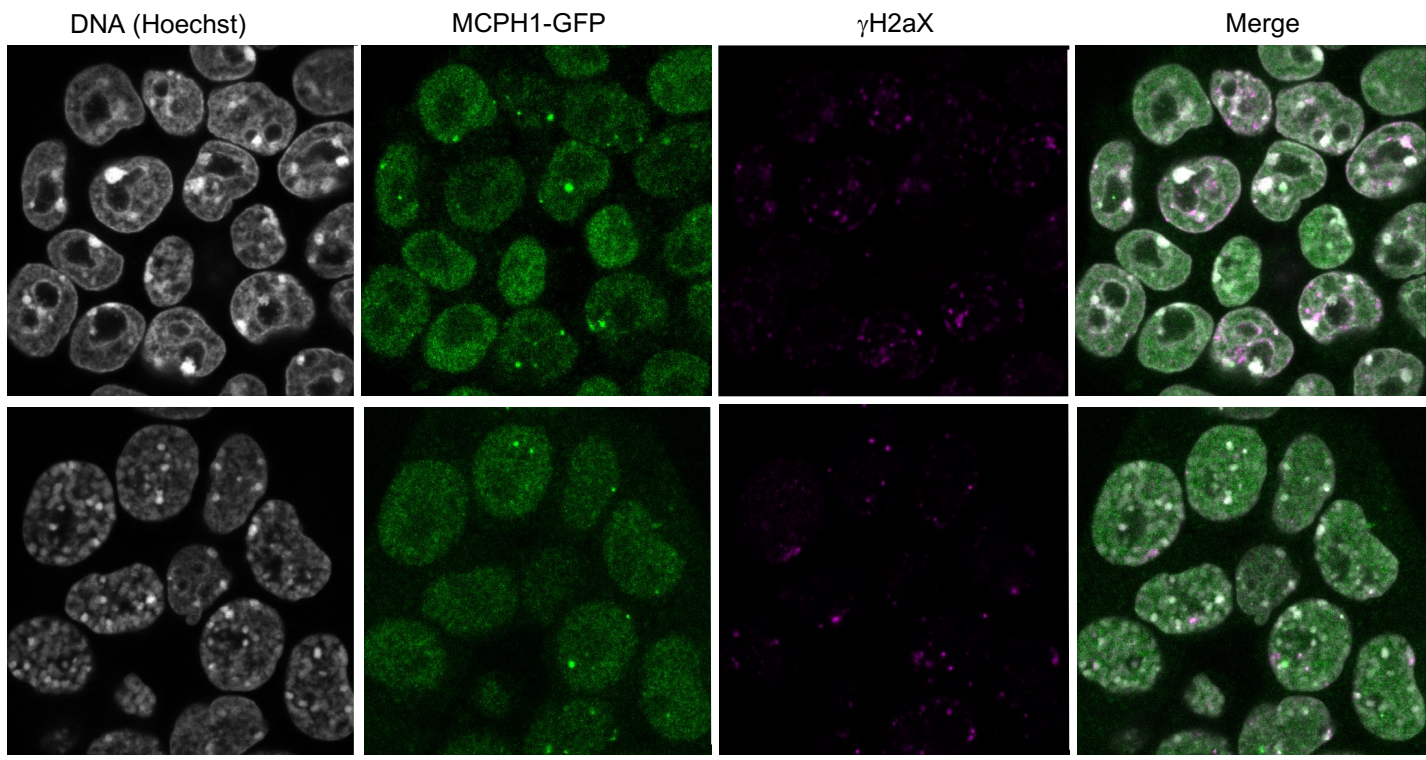

E
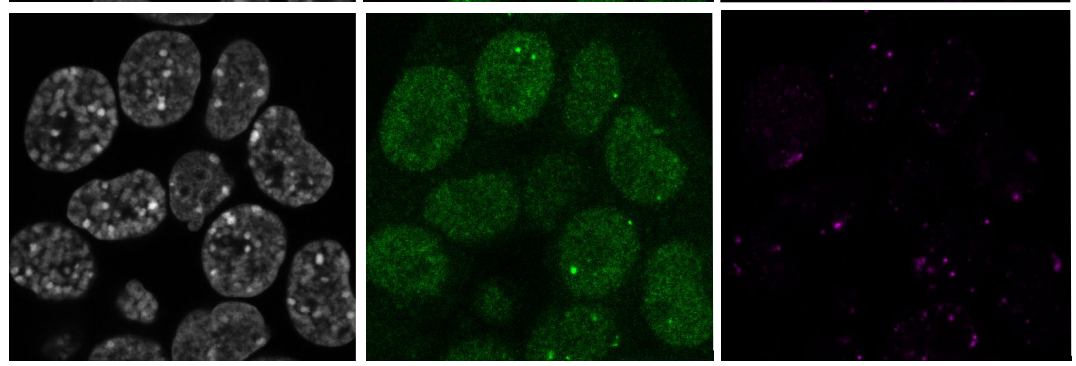

DNA (Hoechst)

MCPH1-GFP

H3PS 10

Merge
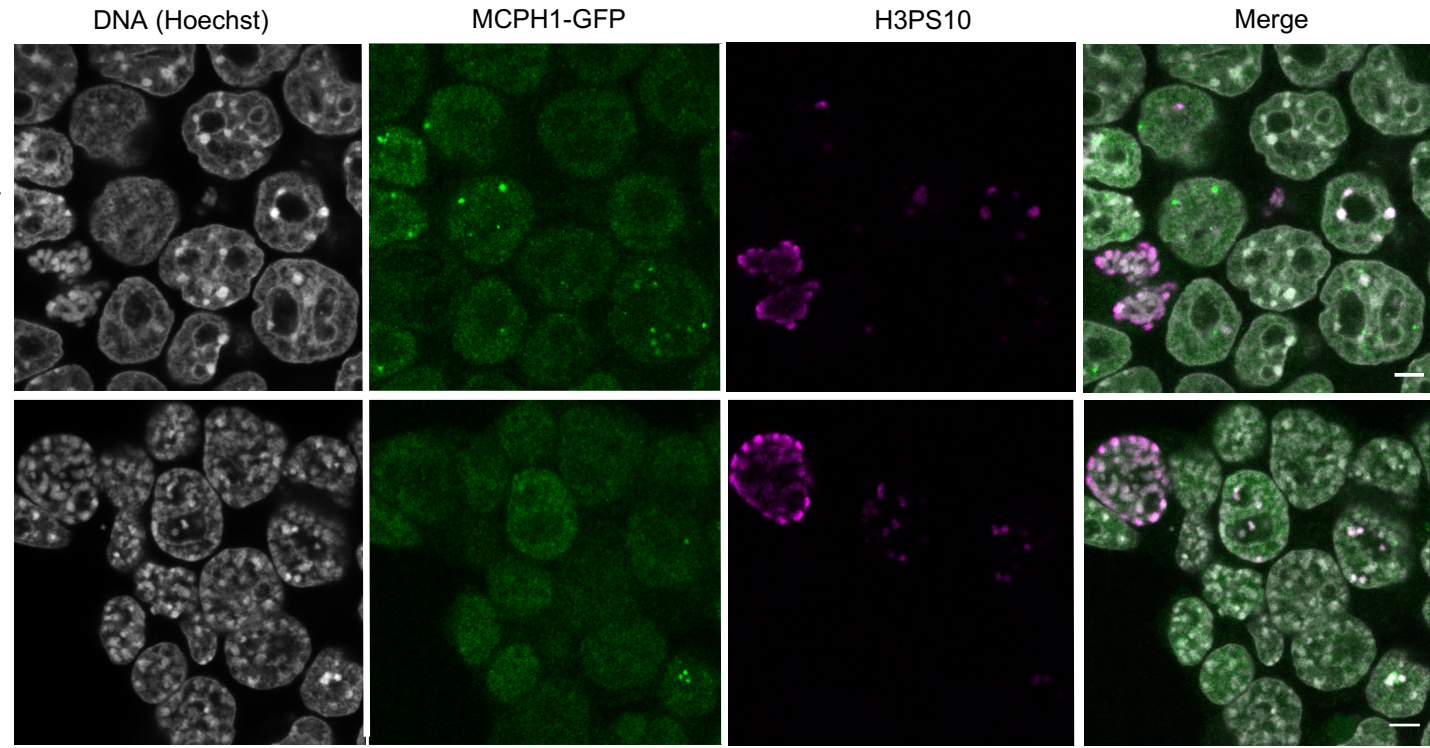


\section{Figure 7}

A

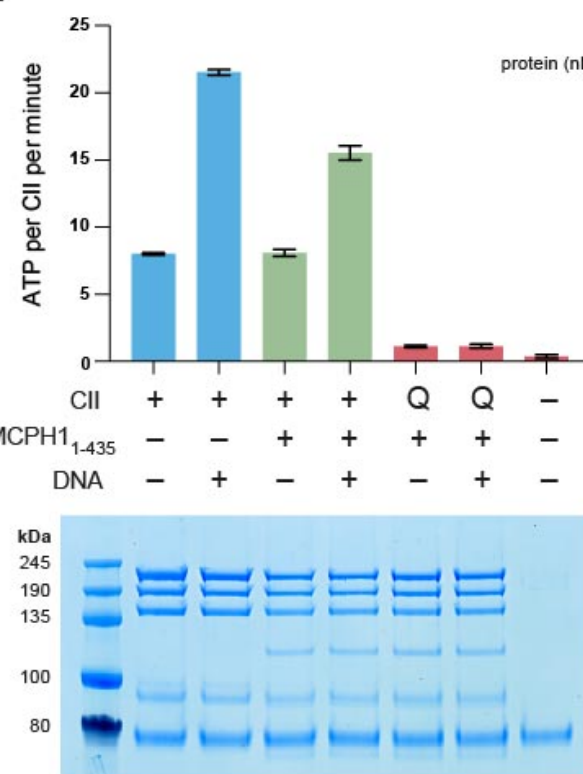

B

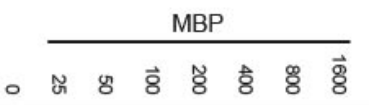

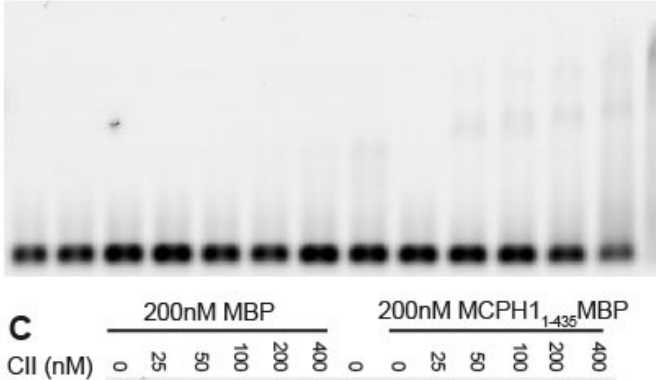

जल $\bar{M}$
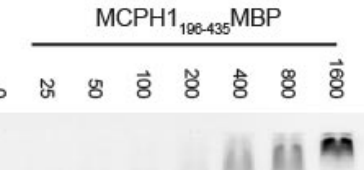
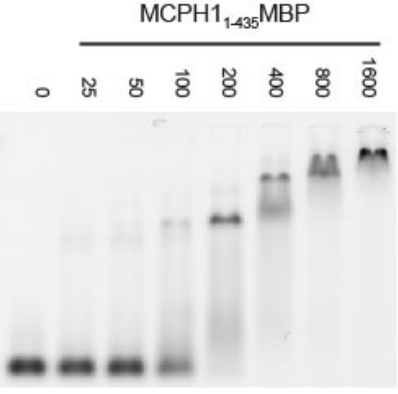

D

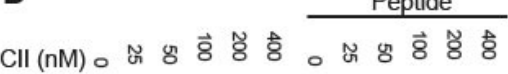

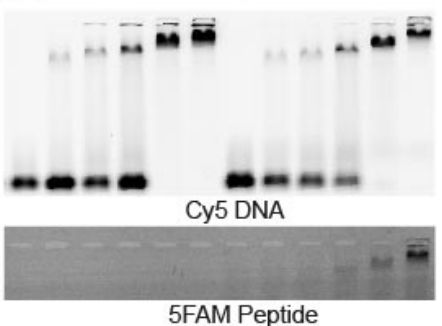




\section{Figure 8}

\section{A}

mRNA Injection:

$+/-\mathrm{MCPH} 1$
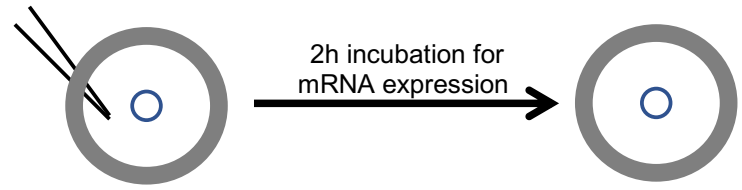

Release

GV Wild type oocyte
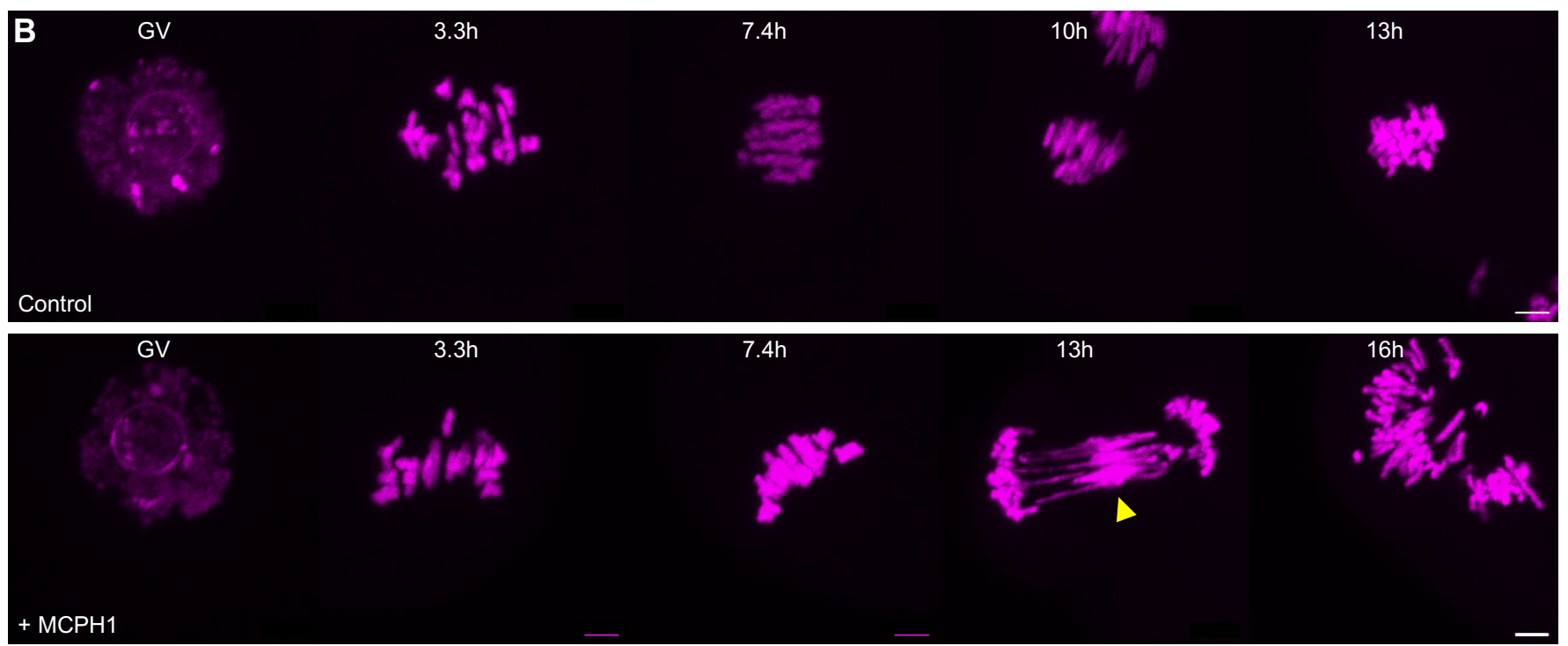

C

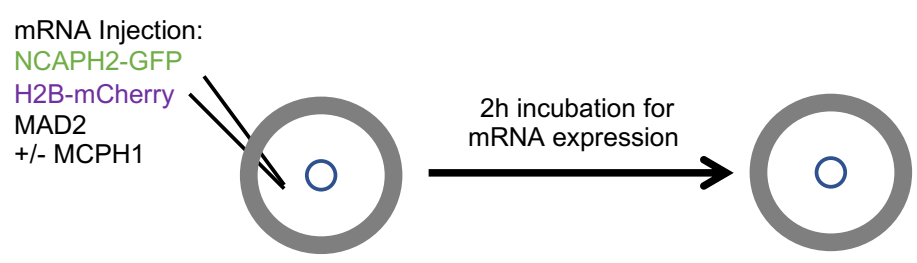

Ncaph2 $2^{\text {lox/lox }}, Z P 3^{\text {TgCre }}$ oocyte

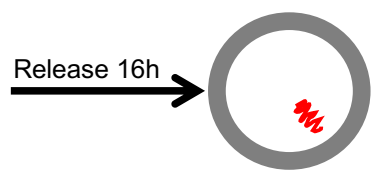

Meiosis I arrested

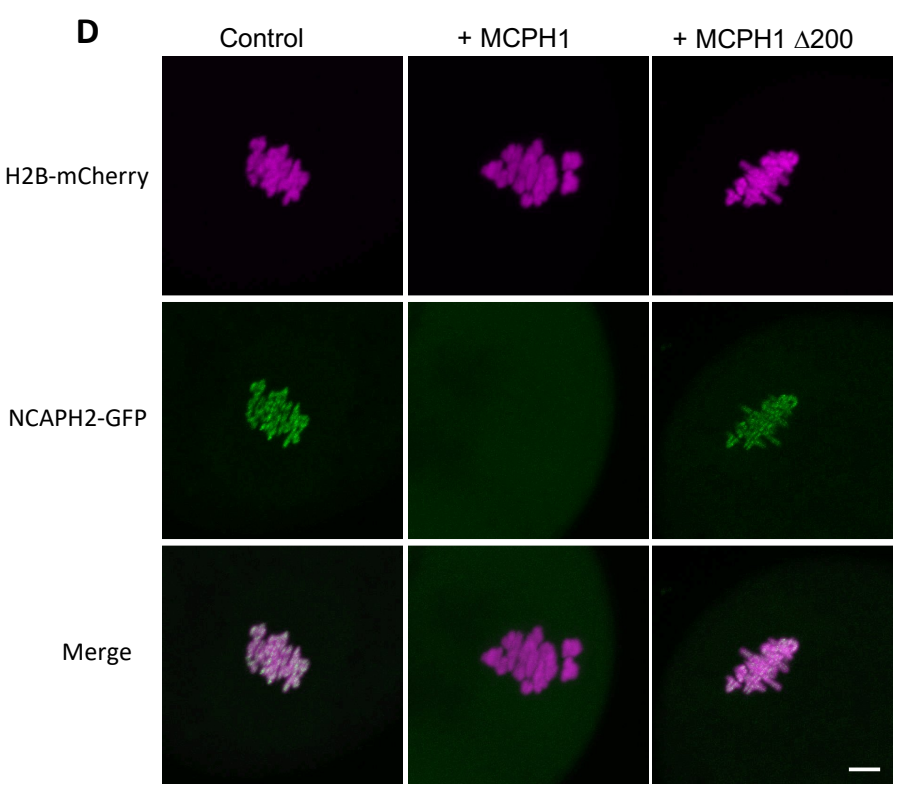

E
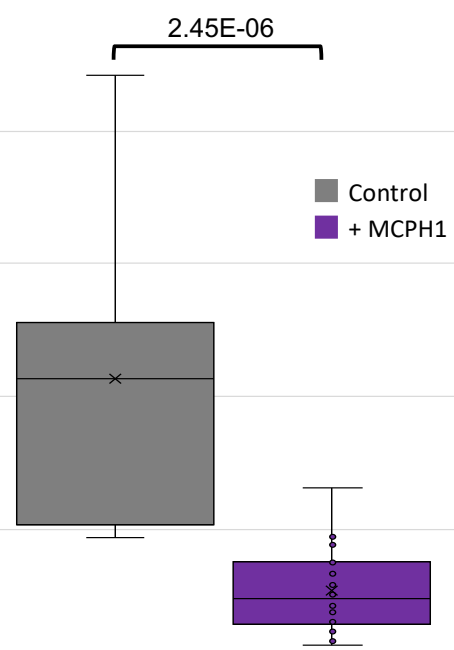
Figure 9

A
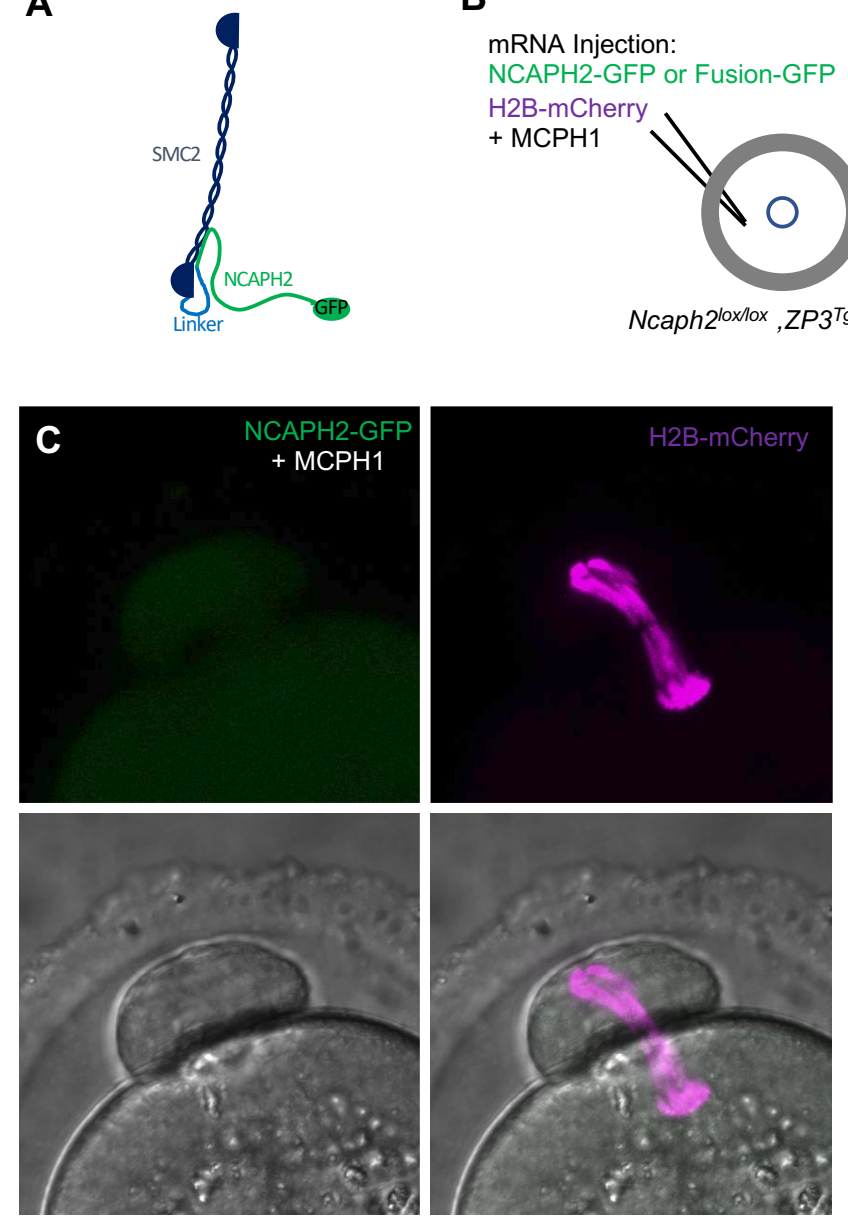

H2B-mCherry
B

mRNA Injection:

NCAPH2-GFP or Fusion-GFP

$+\mathrm{MCPH} 1$
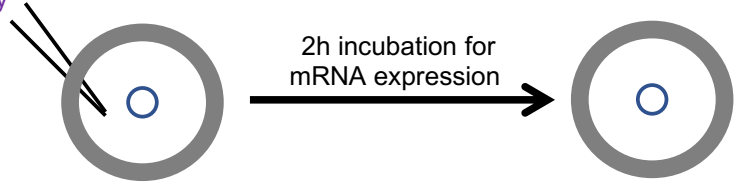

$\stackrel{\text { Release } \longrightarrow \text { Live cell }}{\longrightarrow}$ imaging

Ncaph2/ox/ox ,ZP3'gCre oocyte
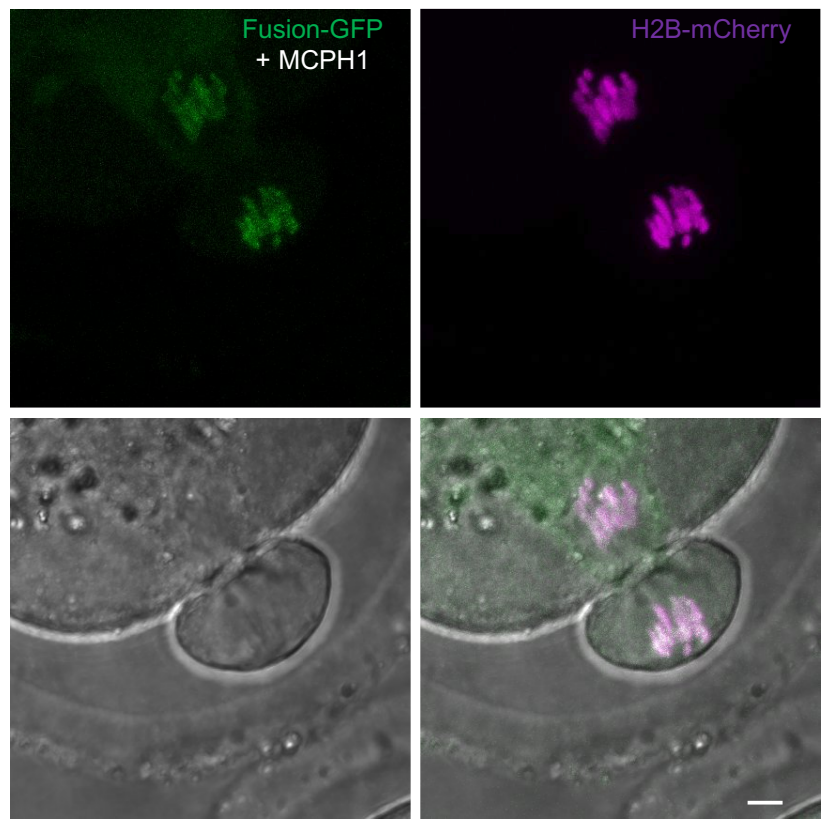

D
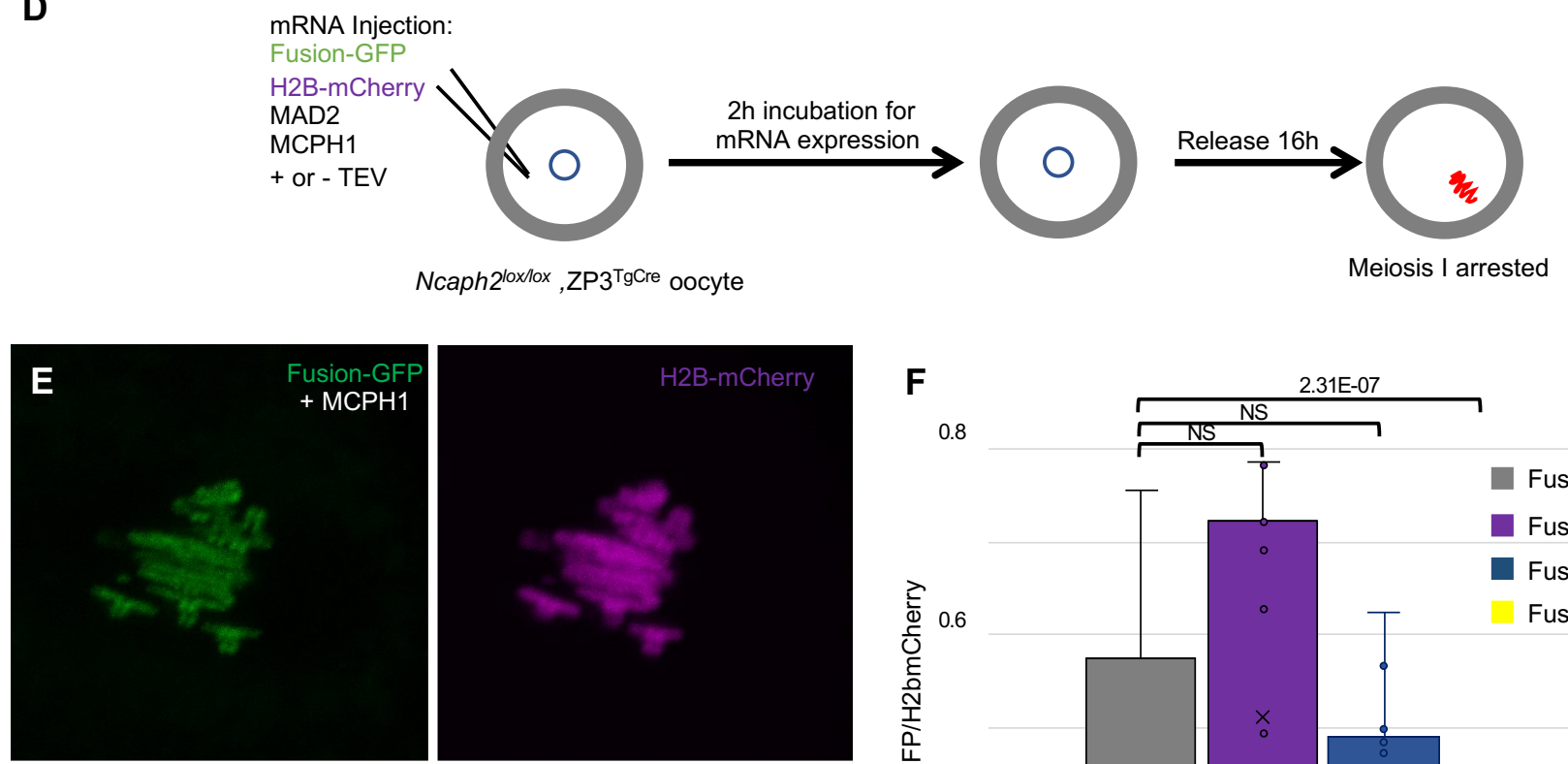

$\mathbf{F}$

Fusion-GFP
$+\mathrm{MCPH} 1$

+ TEV
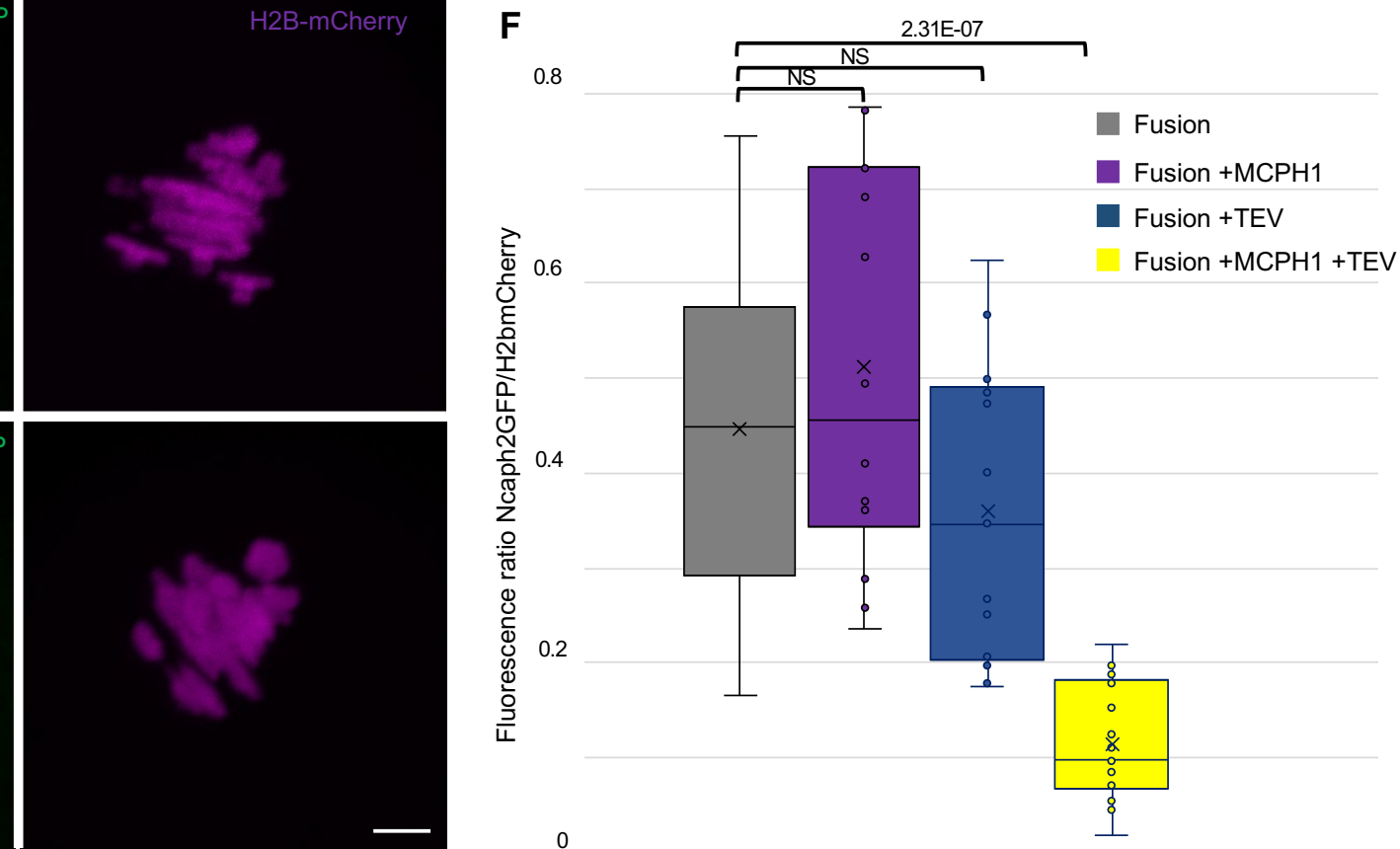
Figure 10

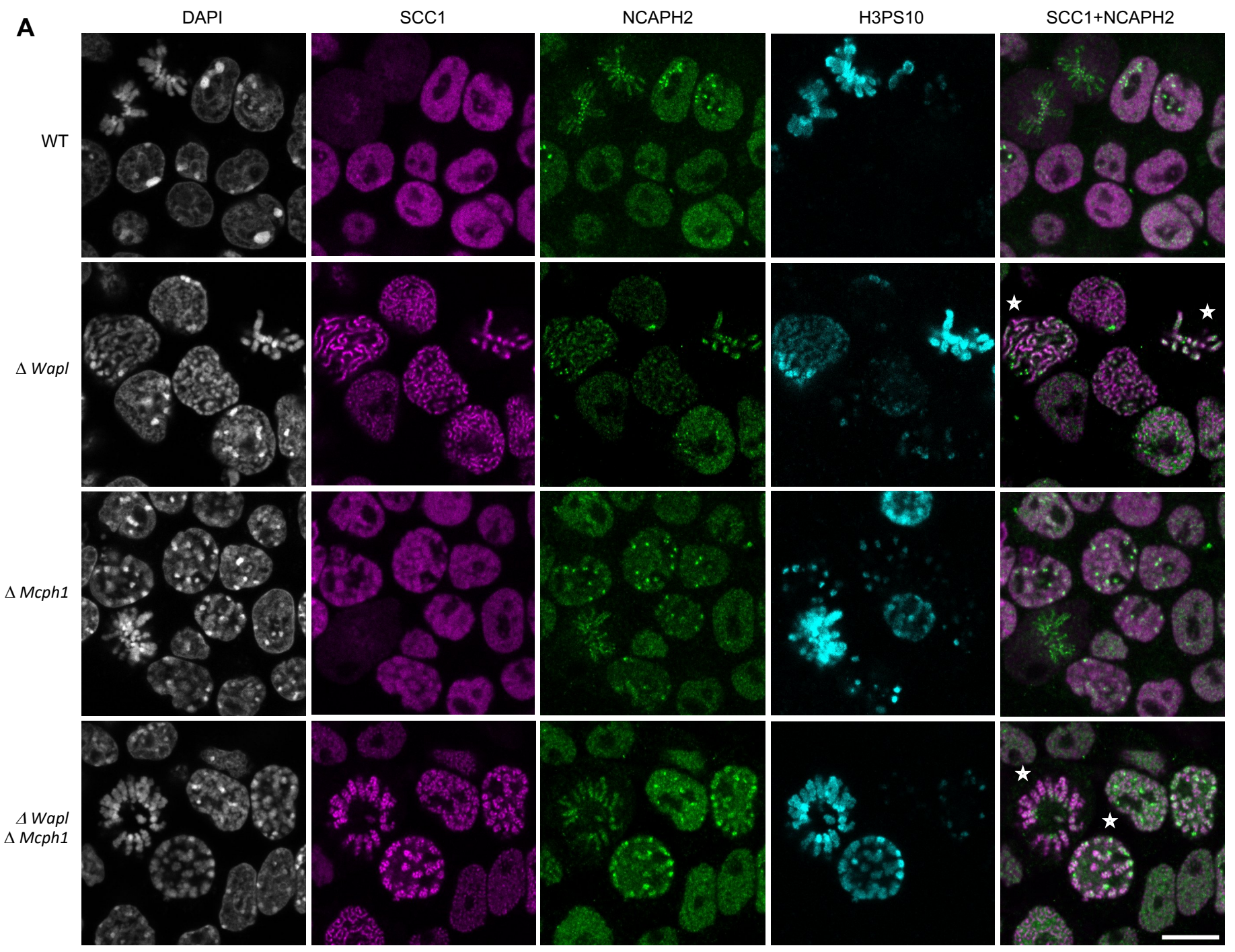

B
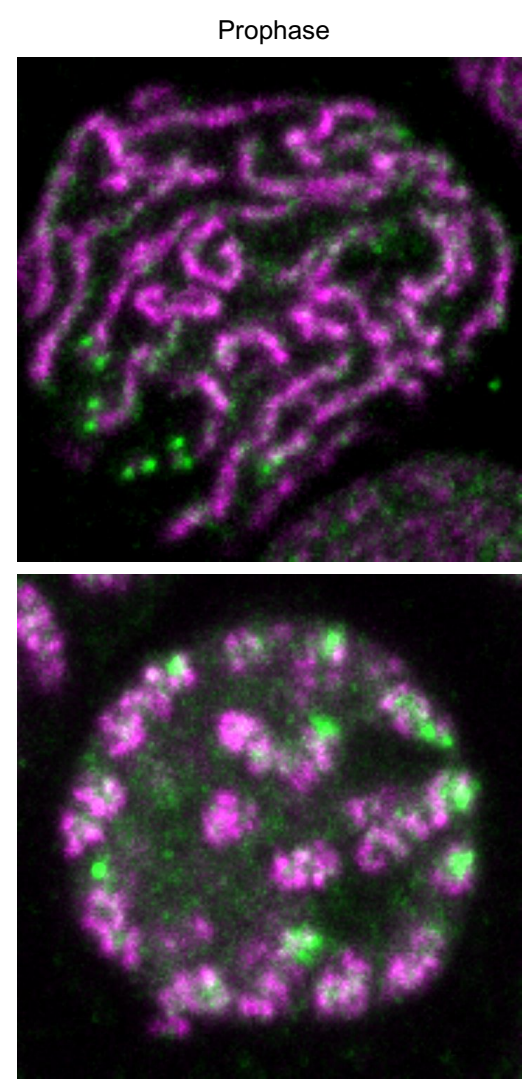

Metaphase
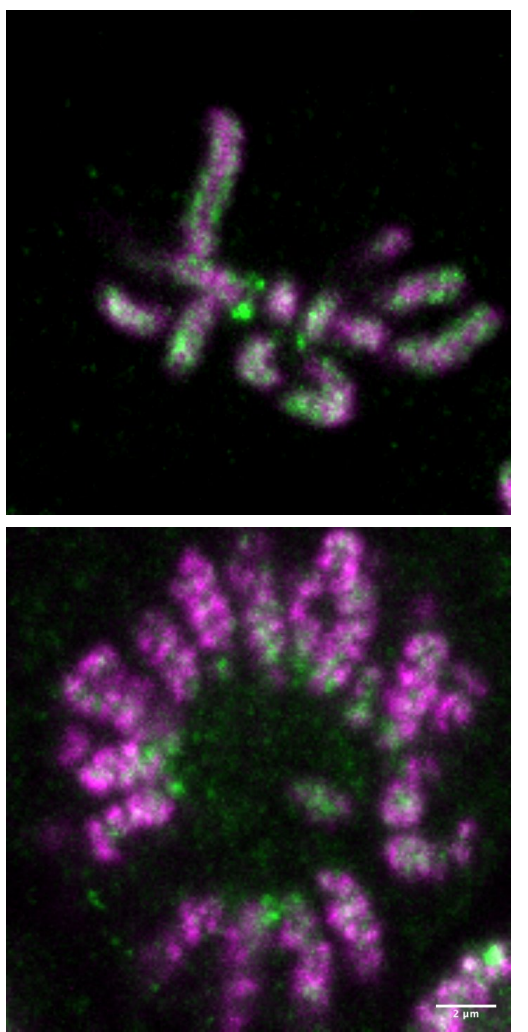
Figure 11

A

G2

M

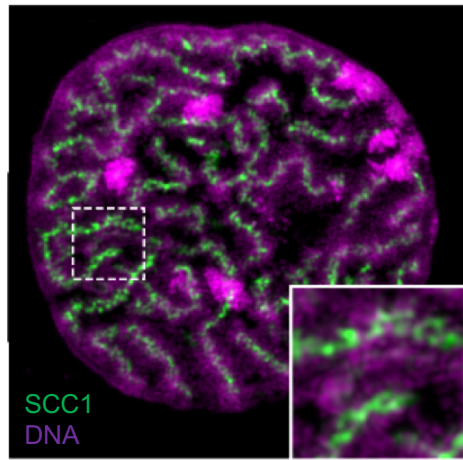

$\Delta W a p l$

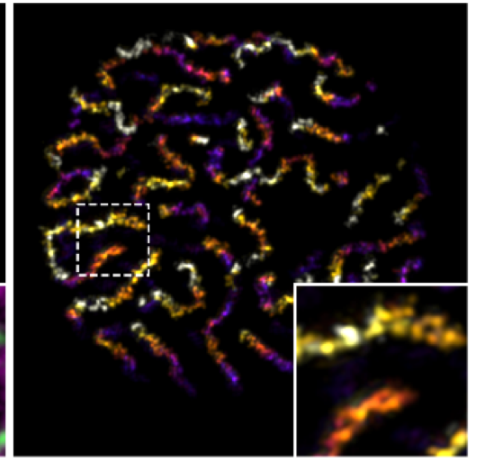

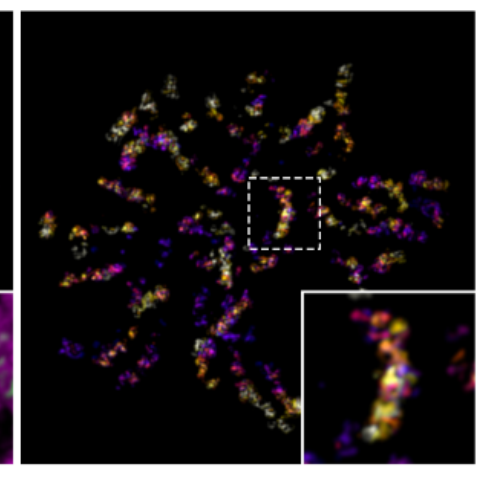

\section{1}

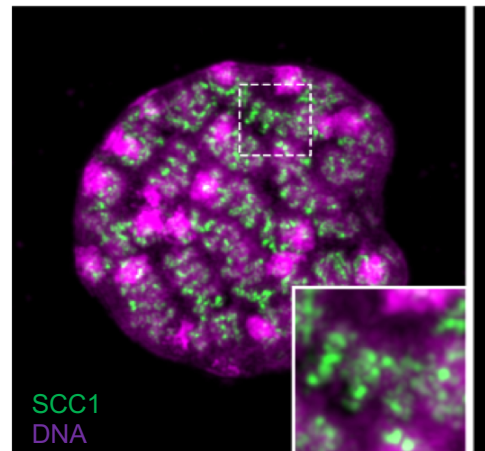

$\Delta$ Wapl, $\Delta M c p h 1$

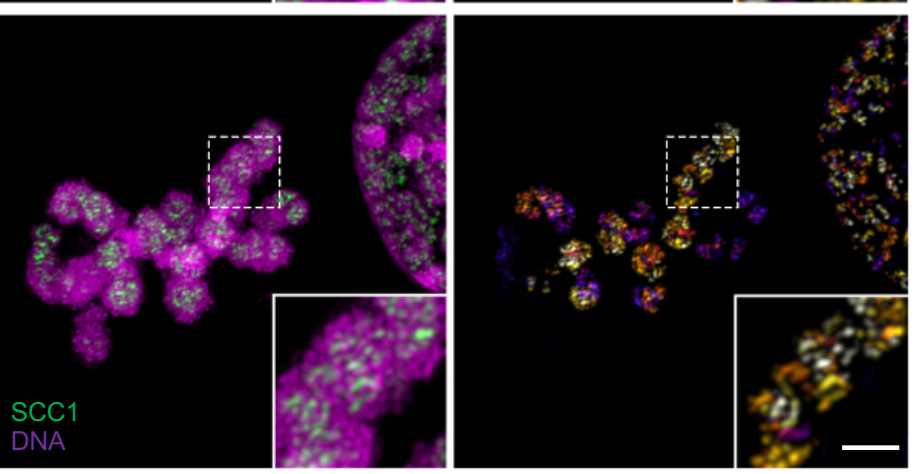

B

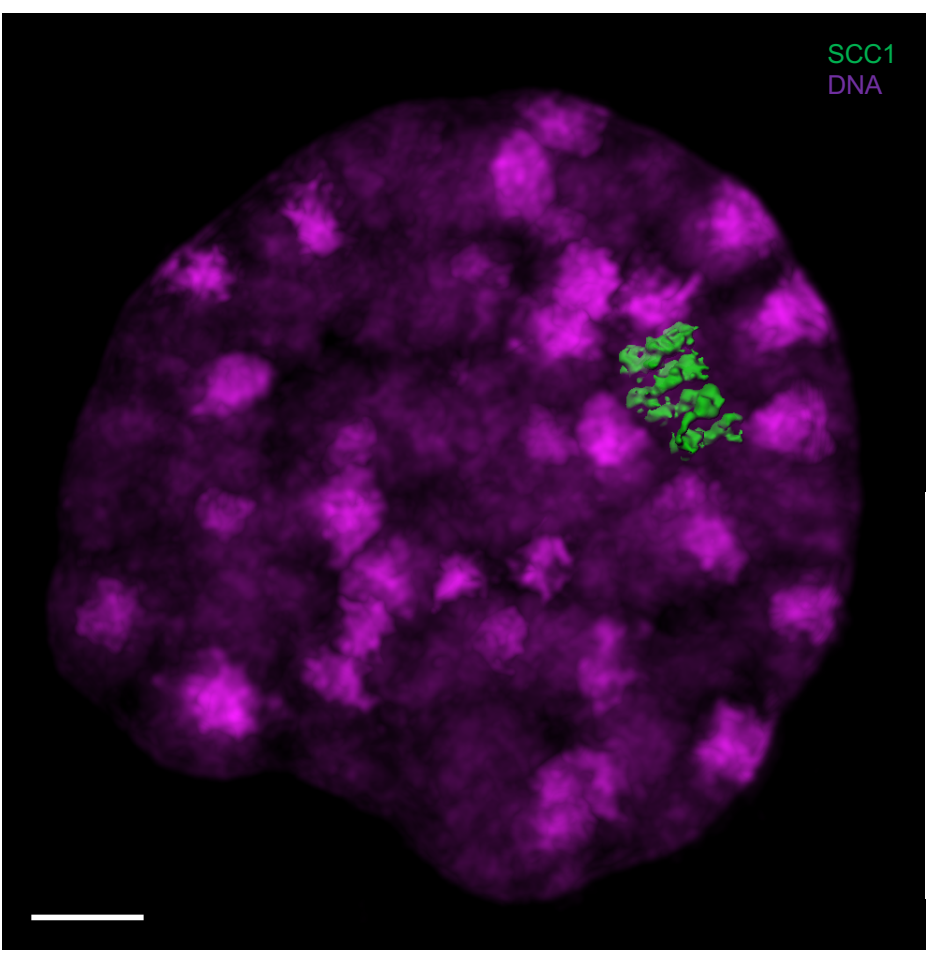

C
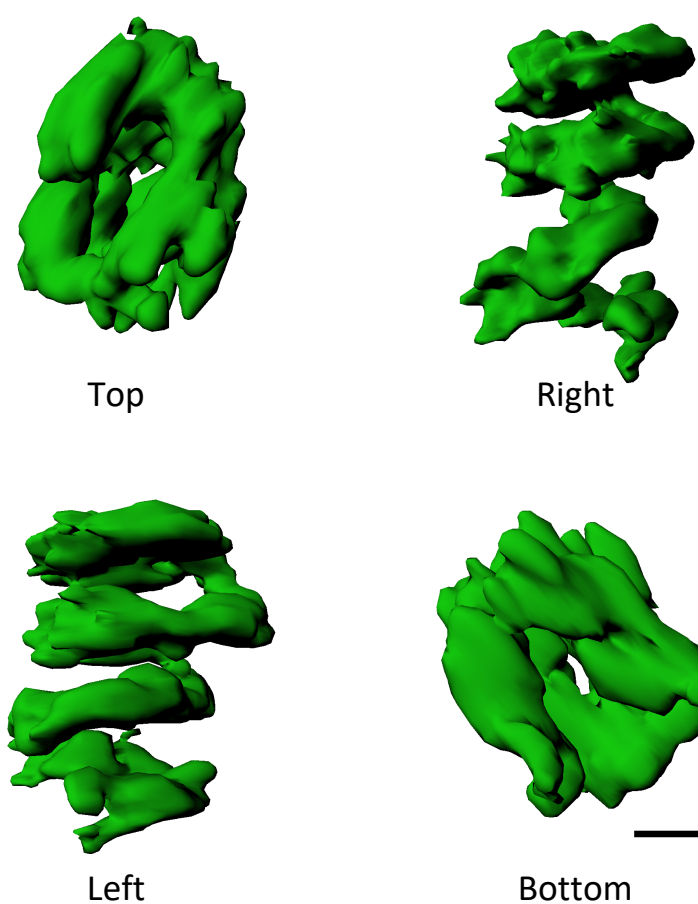

Right

Bottom
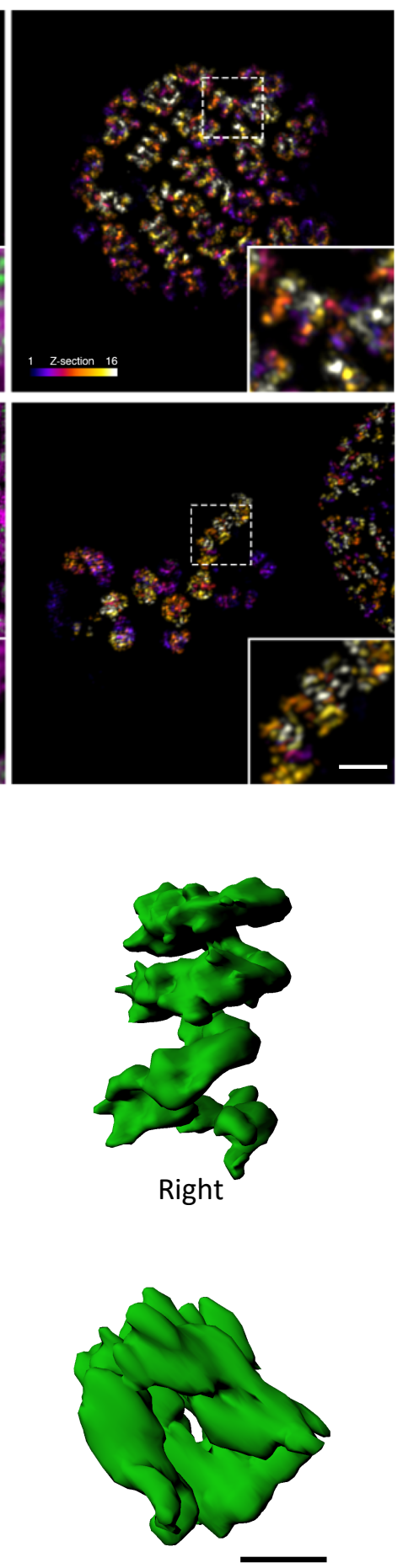


\section{Supplementary Figure 1}

A

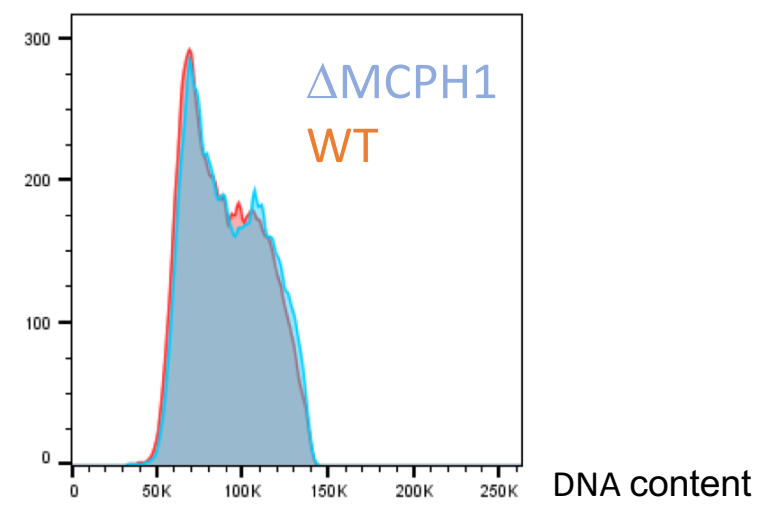

B

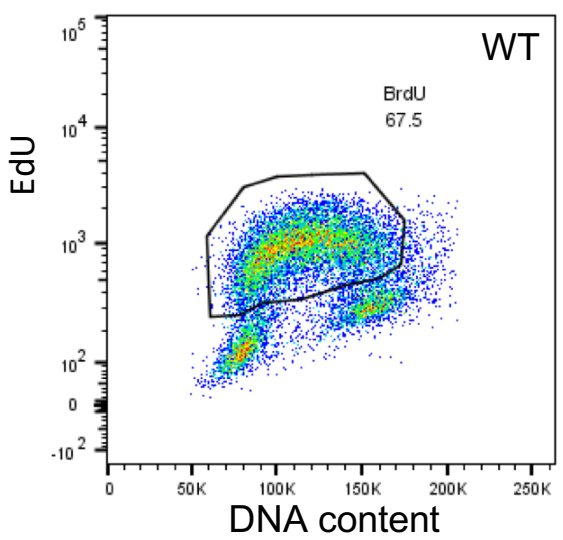

C

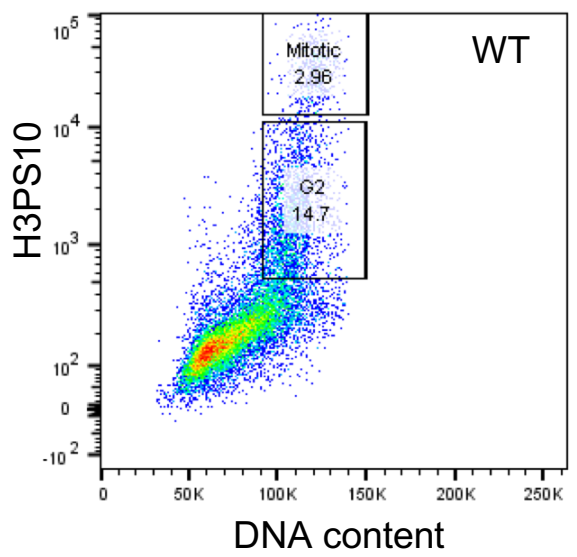

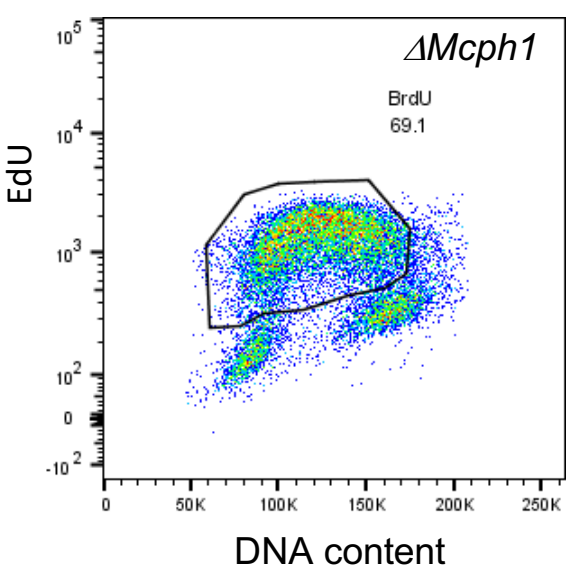

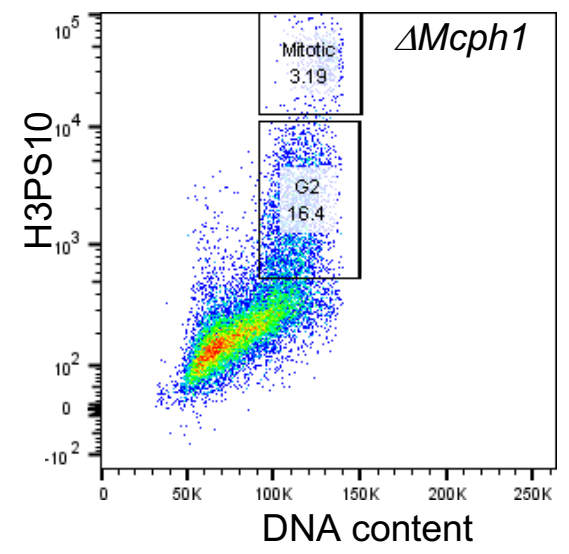

D

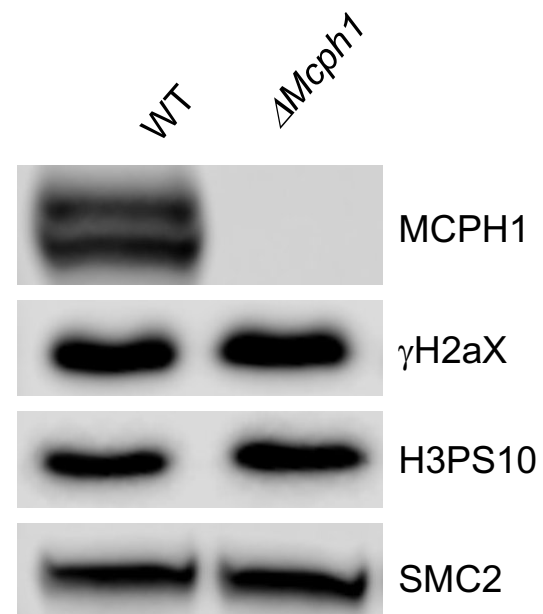




\section{Supplementary Figure 2}

CREST

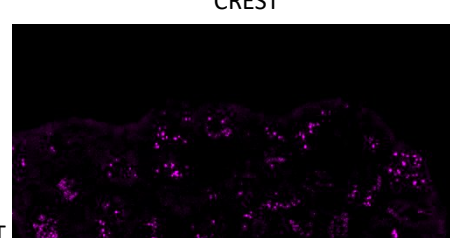

EdU

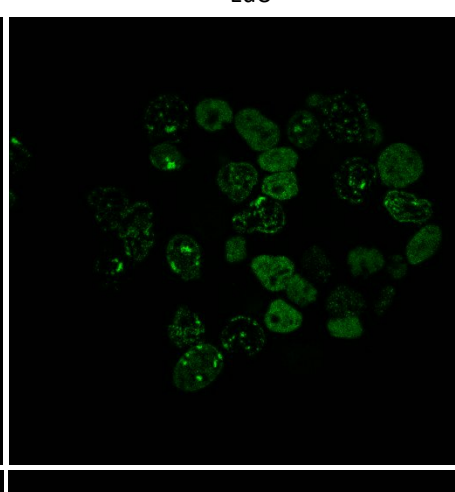

WT

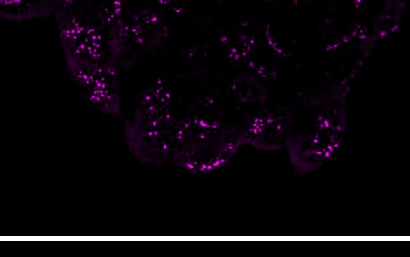

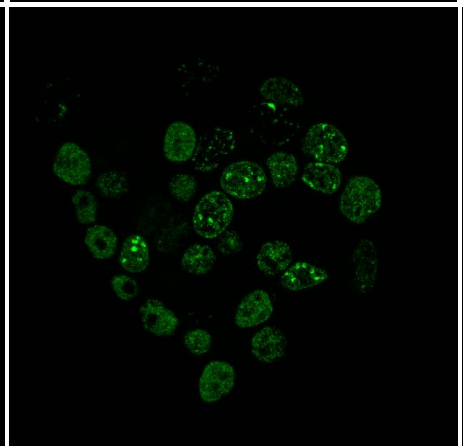

DAPI

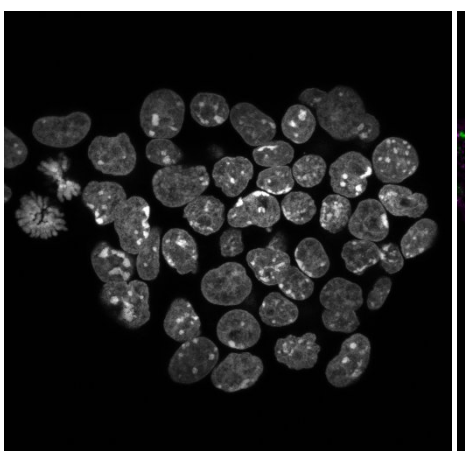

Merge

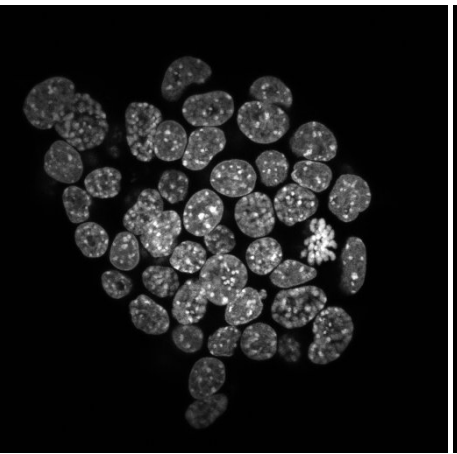

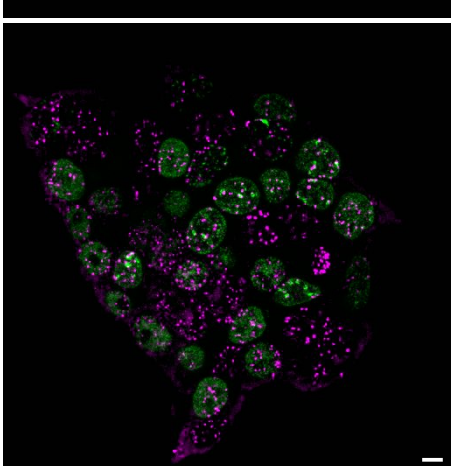




\section{Supplementary Figure 3}

A

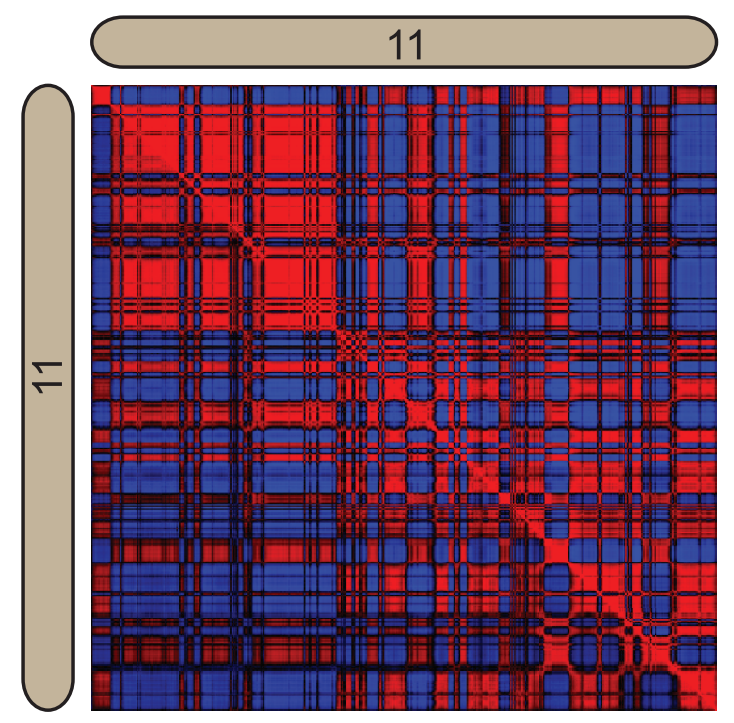

WT
B

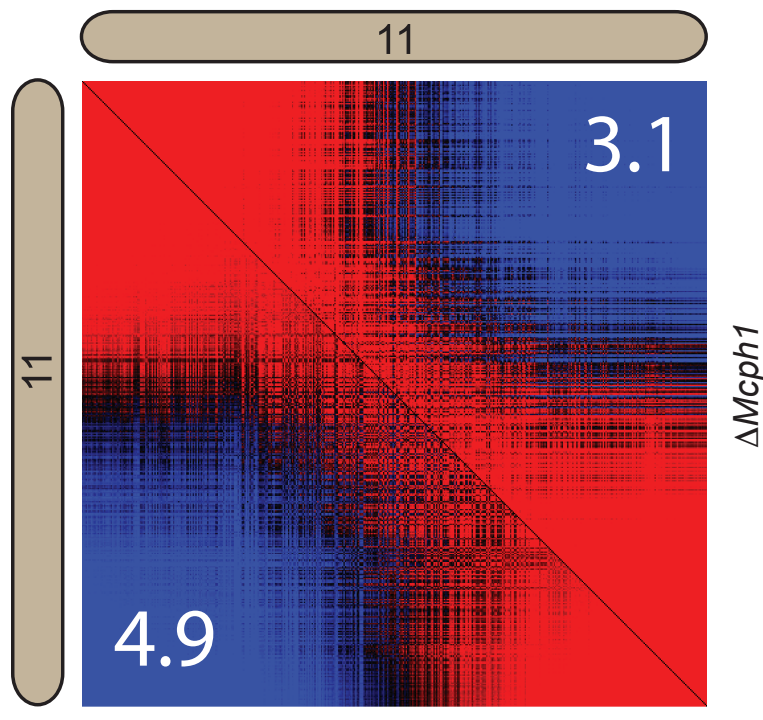

WT

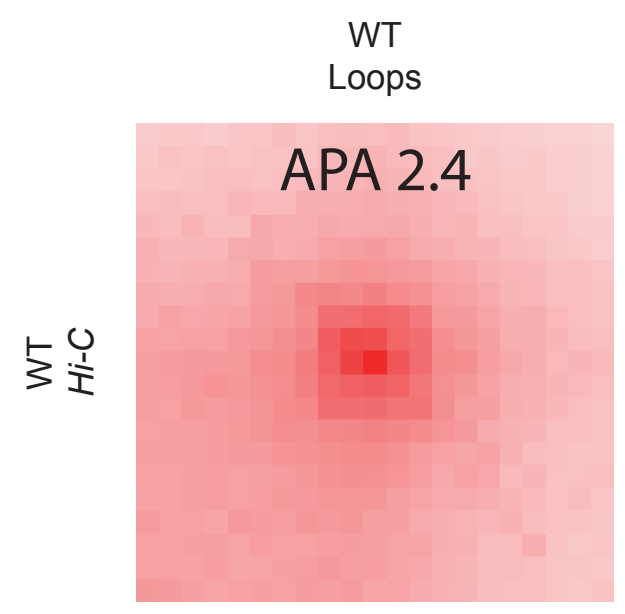

$\Delta$ Mcph1

Loops
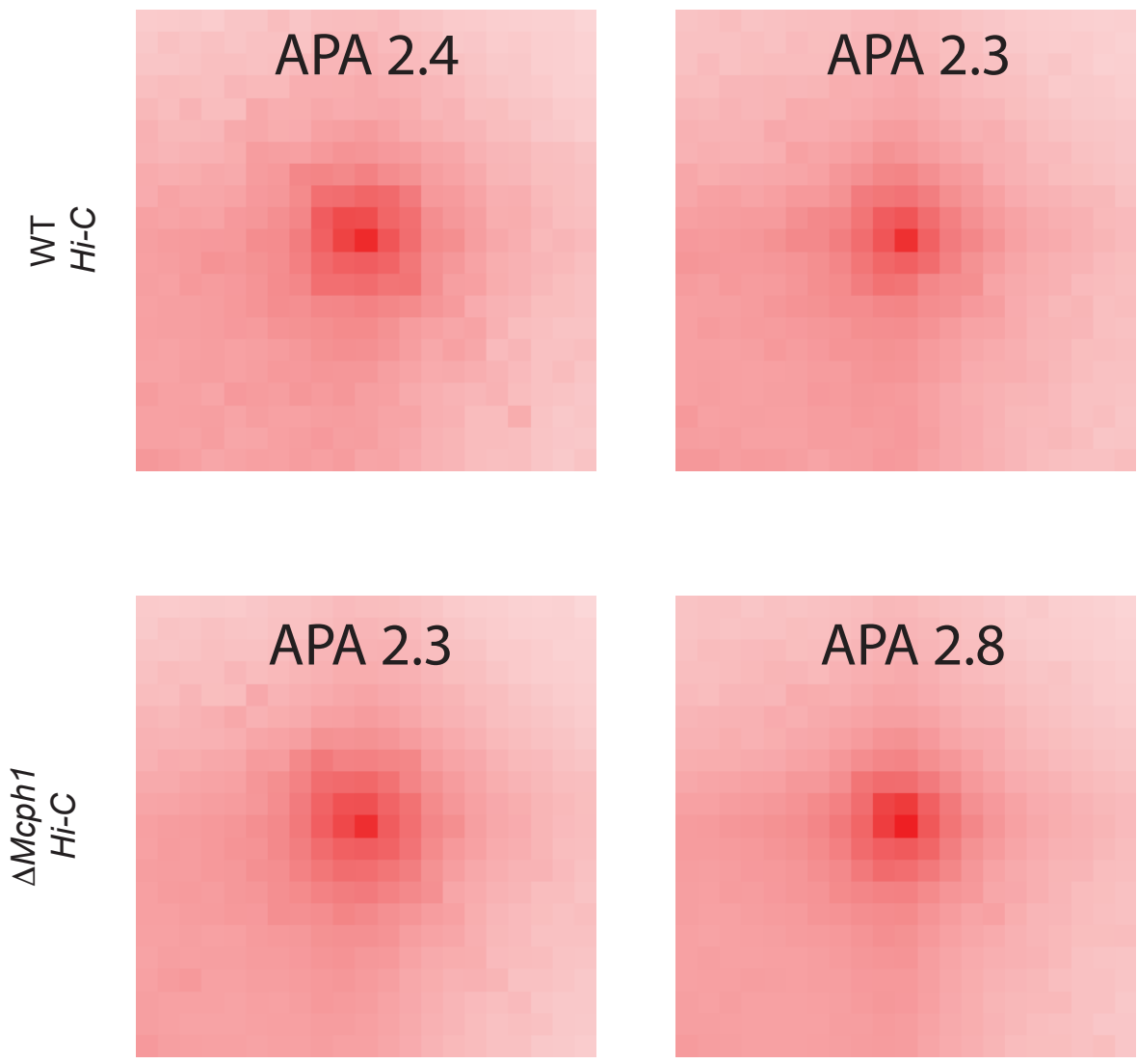
Supplementary Figure 4

A
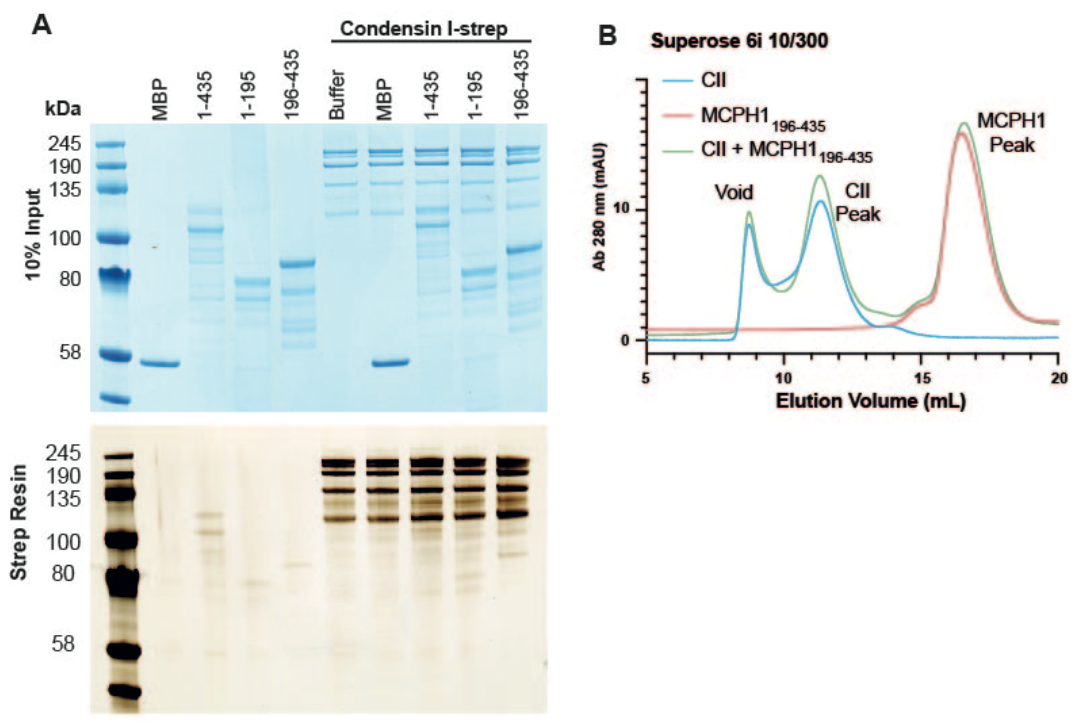

C
1 A P I L K D V $\circ \circ \circ \circ b \circ \circ b b$

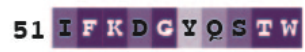

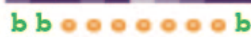

101 S S I I K K K R K C $\circ \circ \mathrm{b} b \circ 00000$

151 I LIFESNGS L $\mathrm{b} b \mathrm{~b} b \circ 0 \circ \circ \mathrm{b}$

201 S H D N P S N S C

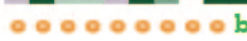

251 L E G S I D I K S

o०० b०o०b०。

301 N I Q R N I A GK V

\section{S S S V KRKRVS}

००००००००。

401 ALEALSCGES
००००००००\%
AYVEVWS SNG

$\mathrm{b}$ b b b b b boo o

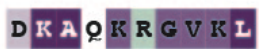

$\circ \circ \mathrm{b} \circ 0.0 \mathrm{~b} \circ \mathrm{b}$

\section{Q P K D F N F K T}

000000000

I Y TPTIEINS

$b$ b b o o b ob o

EAPINISRDT

- $b$ b b o b b ० ०

DVCISSIVIK

- b b o o ० ० b o

VTP D O K

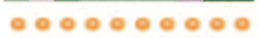

H GS HSP P K E K

०००००००००

\section{SYDDYF SPDN}

\section{TENY SKT TTT}

$\circ \circ \circ \circ b \circ \circ b \mathrm{~b}$ e

\section{S V LWVEKCR}

$\mathrm{b} b \mathrm{~b}$ b b boob o

\section{PENDKRF 9 K K}

R H H S AMEKR L

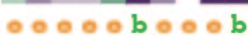

I C SDEYFAGC

$\mathrm{b} b \circ \circ \circ \mathrm{b} \circ \circ \mathrm{b}$

A N N I HS S P S F

००००००००。

MSOETFEEKY

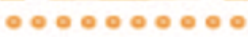

CKRKRSTRRS O०००००००0

\section{IKERYSENL P}

b o ०००००bo

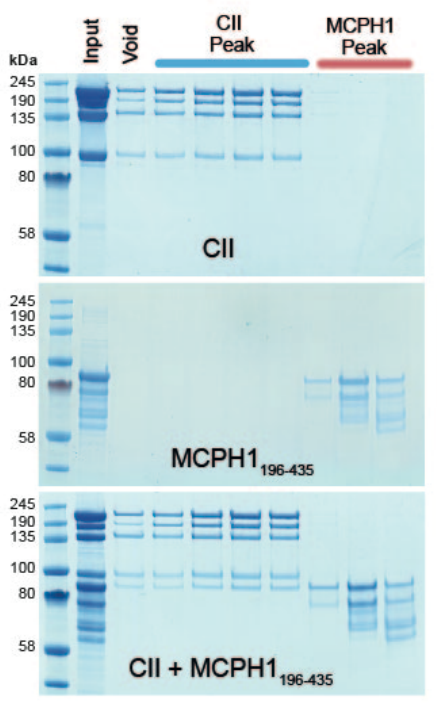

\section{OLVDMGAKVS KTFR OVTHV}

$\circ \mathrm{b} \circ \circ \mathrm{b} \circ \circ \circ \mathrm{b} \circ, \circ \mathrm{b} \circ \circ \circ \mathrm{b} b \circ \mathrm{b}$

TAGAHIDESI FPAANMNEL

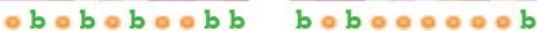

\section{FEKMAKELOR OKTNIDDDVP}

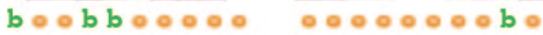

\section{으K KERENL SPTSSOMI

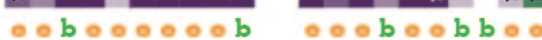

IHSSFDDLCG NSGCGNOERK

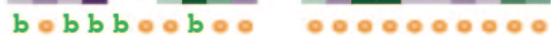

THIDKSSPQK FLSNISKEEI

$00 \mathrm{~b} 0000000$ ०००००00000

\section{RISPTLSSTK GHLLIHSRPR}

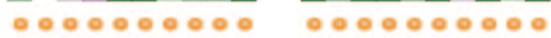

\section{IMPRIQICRS EDRLOHVAGP}

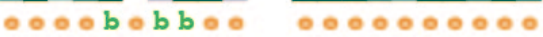

PESOL 435 The conservation scale:

? $\begin{array}{llllllllll}1 & 2 & 3 & 4 & 5 & 6 & 7 & 8 & 9\end{array}$ 
Supplementary Figure 5

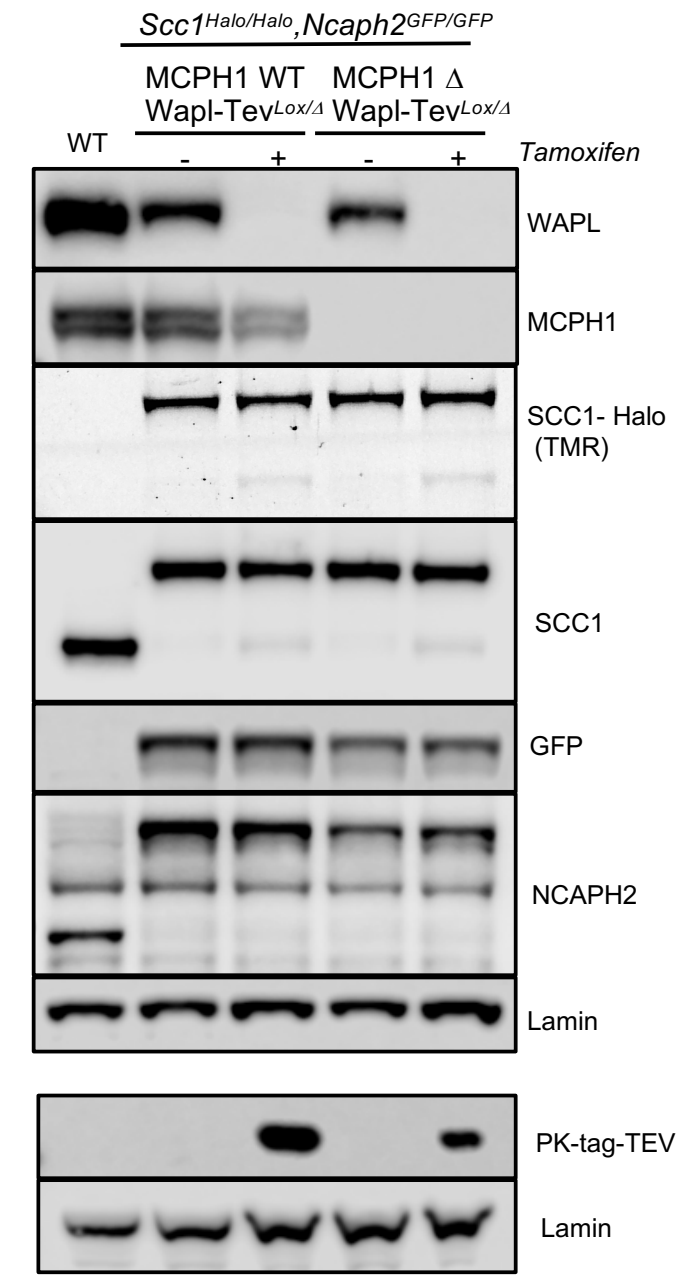

